\title{
Review Article \\ On the Nature of Electronic Wave Functions in One-Dimensional Self-Similar and Quasiperiodic Systems
}

\author{
Enrique Maciá \\ Departamento de Física de Materiales, Facultad de CC. Físicas, Universidad Complutense de Madrid, 28040 Madrid, Spain \\ Correspondence should be addressed to Enrique Maciá; emaciaba@fis.ucm.es
}

Received 3 September 2013; Accepted 26 November 2013; Published 30 March 2014

Academic Editors: A. N. Kocharian, E. Liarokapis, and A. Oyamada

Copyright (C) 2014 Enrique Maciá. This is an open access article distributed under the Creative Commons Attribution License, which permits unrestricted use, distribution, and reproduction in any medium, provided the original work is properly cited.

\begin{abstract}
The interest in the precise nature of critical states and their role in the physics of aperiodic systems has witnessed a renewed interest in the last few years. In this work we present a review on the notion of critical wave functions and, in the light of the obtained results, we suggest the convenience of some conceptual revisions in order to properly describe the relationship between the transport properties and the wave functions distribution amplitudes for eigen functions belonging to singular continuous spectra related to both fractal and quasiperiodic distribution of atoms through the space.
\end{abstract}

\section{Basic Notions}

1.1. Orderings of Matter. The notion of order is one of the most fundamental ones. In fact, order inspires the best human civilization achievements in politics, ethics, arts, and sciences [1]. Order pervades also most workings of Nature as the universe unfolds creating symmetric patterns and stable structures. Among them, solid matter arrangements were initially categorized in a dichotomist way, namely, as either ordered or disordered matter forms. In this way, ordered matter was identified with periodic arrays of atoms through the three-dimensional space, while disordered matter was related to random atomic distributions instead. Thus, the notions of crystalline matter and spatial periodicity were born interwoven from the very beginning, just as amorphous matter was conceptually related to randomness in a natural way (Figure 1(a)).

Nevertheless, the unexpected finding of incommensurate phases during the 1960s and 1970s, followed by the discovery of quasicrystalline alloys in 1982, opened up a discussion forum on the very crystal notion in the crystallographic, condensed matters physics and materials science communities. Indeed, initially it was thought that quasicrystals (short for quasiperiodic crystals) corresponded to a somewhat intermediate order form between that of crystals and amorphous materials [2]. However, it was soon realized that quasicrystals (QCs), exhibiting long-range order along with orientational symmetries not compatible with periodic translations, actually represented a new order style, which should be properly interpreted as a natural extension of the notion of a crystal to structures with quasiperiodic (QP), instead of periodic, arrangements of atoms [3]. Consequently, the International Union of Crystallography widened in 1992 the very definition of crystal, introducing two separate categories of crystal representatives referred to as periodic and aperiodic crystals, respectively. According to the proposed terms of reference:

In the following by "crystal" we mean any solid having an essentially discrete diffraction diagram, and by "aperiodic crystal" we mean any crystal in which three-dimensional lattice periodicity can be considered to be absent [4].

Thus, QCs along with incommensurate phases belong to the novel aperiodic crystals category, whereas usual periodic crystals are now known as classical crystals (Figure 1(b)). The revamped crystal definition reflects our current understanding that microscopic periodicity is a sufficient but not necessary condition for crystallinity. Therefore, the presence of a mathematically well-defined, long-range atomic order should be regarded as the generic attribute of crystalline matter rather than mere periodicity. At the same time, the essential attribute of crystallinity is transferred from real space to reciprocal space through the recourse to the diffraction patterns, hence highlighting the importance of 
(a) Before 1992

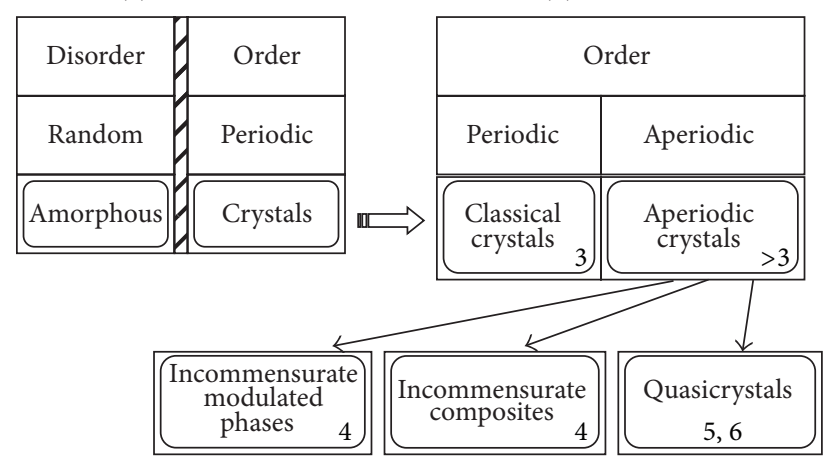

FIGURE 1: In 1992 the notion of crystal was widened beyond mere periodicity. This conceptual diagram presents the position of aperiodic crystals, no longer based on the notion of periodic translation symmetry, among the different orderings of matter. The diverse aperiodic crystal families are arranged according to the dimension $D$ (see (2)) of their embedding hyperspaces (numerical labels).

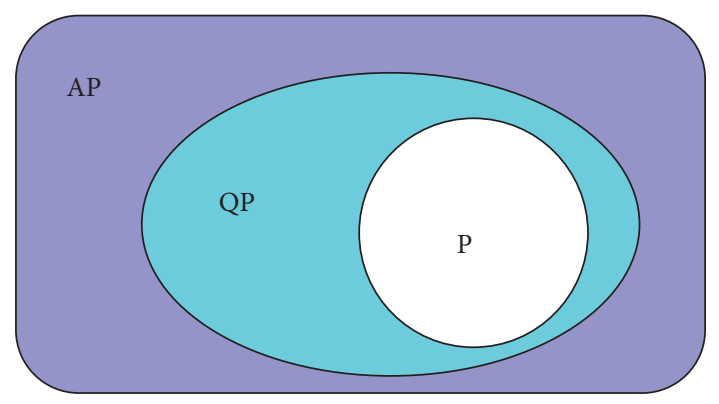

FIGURE 2: Graphical representation of the hierarchical nested relationship among almost periodic (AP), quasiperiodic $(\mathrm{QP})$, and periodic $(\mathrm{P})$ functions.

the Fourier transform in order to properly analyze atomic density distributions.

Despite the fact that more than two decades have elapsed since the crystal notion has been properly revisited one can still find in the literature a lot of works plainly stating that quasiperiodic systems (QPS) provide an example of intermediate structures between ordered and disordered systems. Sentences like this certainly rely on a too vague notion of the term "intermediate" which apparently ignores the fact that every QP function can be expressed in terms of a numerable set of periodic functions in an appropriate high-dimensional space. Accordingly, periodic functions are but the simpler particular instances of the more general QP ones. From this perspective, QPS are not only perfectly ordered structures, but they may even be regarded as having a higher order degree than periodic ones. This viewpoint is nicely illustrated by the hierarchical relationship between almost periodic (AP), $\mathrm{QP}$, and periodic functions shown in Figure 2. Indeed, from a mathematical viewpoint periodic functions are a special case of QP functions which are, in turn, a special case of AP functions. ${ }^{1}$
Therefore, rather than adopting the old dichotomist way (which only allows one to get fuzzy qualitative comparisons as to whether a particular system is less random or more periodic than any other one), it may be more fruitful to think in terms of the different hierarchies of order to which these systems belong (see Figure 7).

Almost periodic functions can be uniformly approximated by Fourier series containing a countable infinity of pairwise incommensurate reciprocal periods (frequencies) $[5,6]$. When the set of reciprocal periods (frequencies) required can be generated from a finite-dimensional basis, the resulting function is referred to as a QP one. ${ }^{2}$ For the sake of illustration, let us consider an aperiodic crystal whose atomic distribution is given by a QP function expressed in terms of its discrete Fourier decomposition:

$$
f(\mathbf{x})=\sum_{\mathbf{k}} a_{\mathbf{k}} e^{i \mathbf{k} \cdot \mathbf{x}},
$$

where the reciprocal vectors are defined by

$$
\mathbf{k}=\sum_{j=1}^{N} n_{j} \mathbf{b}_{j}
$$

where $\mathbf{b}_{j}$ are reciprocal lattice basis vectors. If the minimal number of these basis vectors is larger than three, that is, $N>3$ in (2), then a higher dimensional description is needed to describe the reciprocal lattice, and the related structure is an aperiodic crystal. Otherwise, we obtain a periodic crystal (Figure 1(b)).

The simplest one-dimensional example of a QP function can be written as

$$
f(x)=A_{1} \cos (x)+A_{2} \cos (\alpha x),
$$

where $\alpha$ is an irrational number and $A_{1}$ and $A_{2}$ are real numbers. It is interesting to note that this $\mathrm{QP}$ function can be obtained as a one-dimensional projection of a related periodic function in two dimensions:

$$
f(x, y)=A_{1} \cos x+A_{2} \cos y,
$$

through the restriction $y=\alpha x$. This property is at the basis of the so-called cut and project method, which is widely used in the study of QCs. In fact, since any QP function can be thought of as deriving from a periodic function in a space of higher dimension, most of the basic notions of classical crystallography can be properly extended to the study of QCs in appropriate hyperspaces $[5,7]$.

1.2. Extended, Localized, and Critical Wave Functions. Once we have clarified that aperiodic crystals do not occupy a vague intermediate position between periodic crystal and amorphous matter representatives, it is pertinent to indicate that there exists a physical context in which one can properly talk about the existence of an intermediate state between order and disorder. This scenario is that occurring when a system undergoes a phase transition from solid to liquid states, experiencing critical fluctuations at all scales. This 


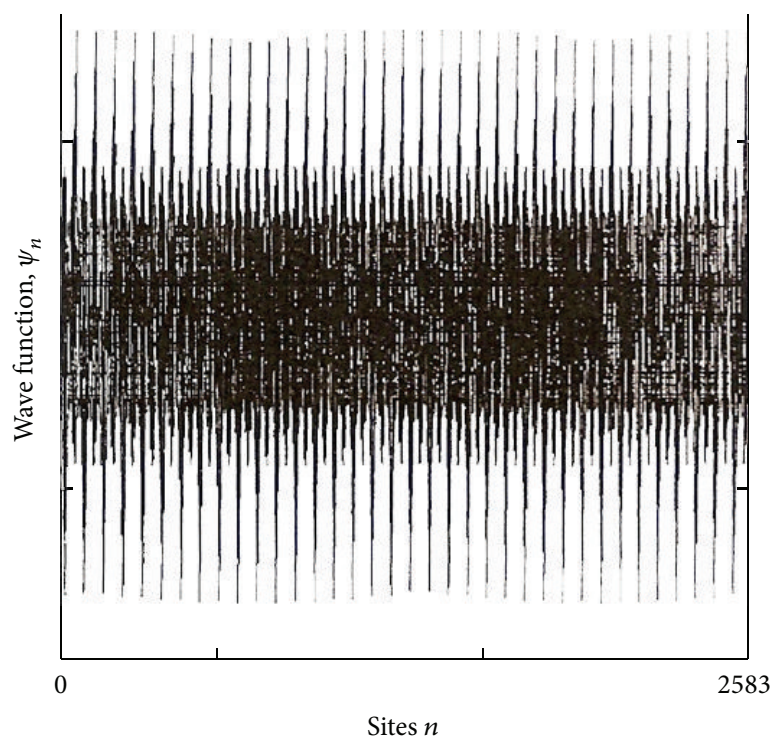

(a)

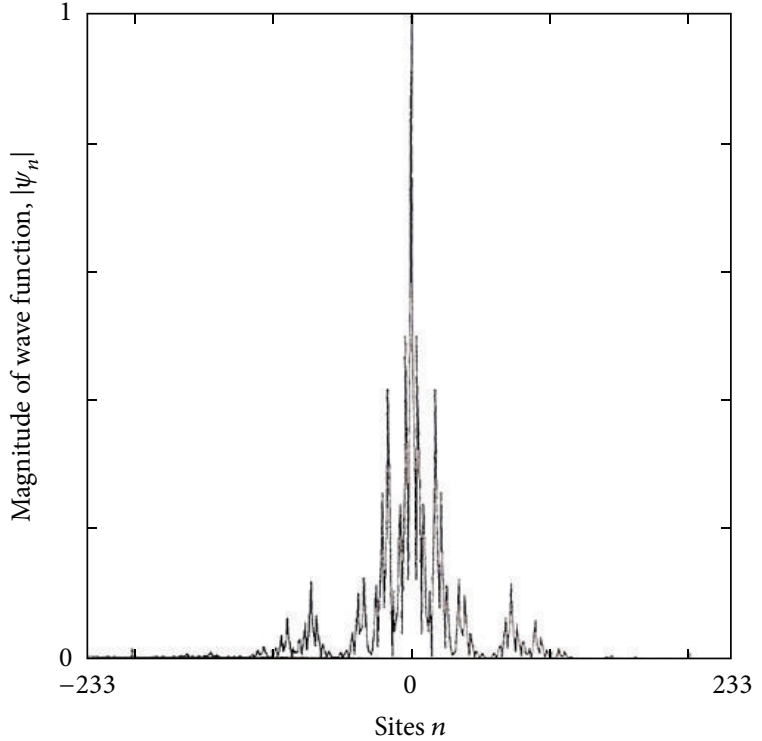

(b)

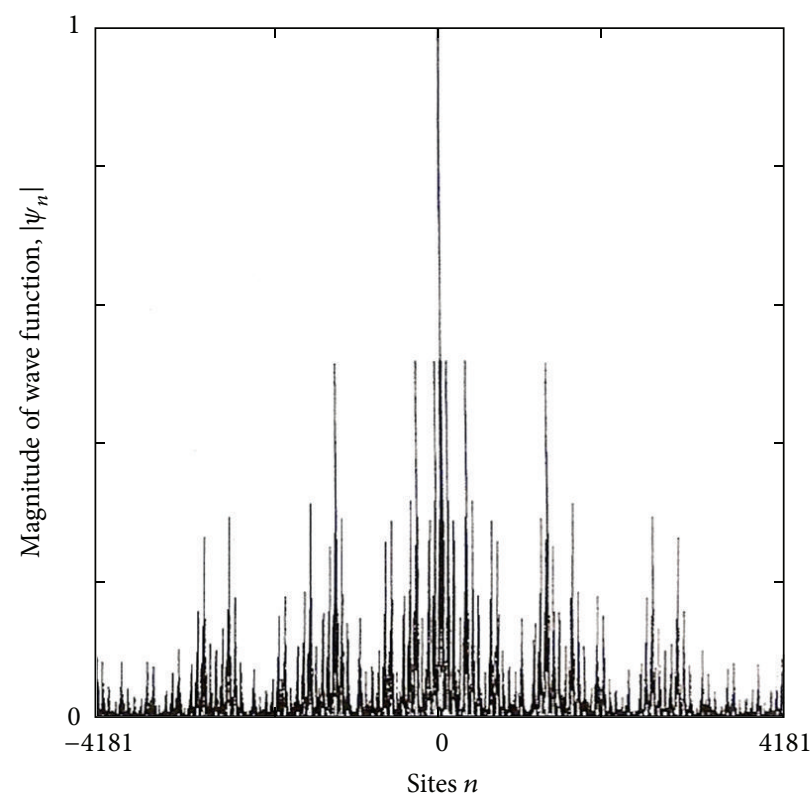

(c)

FIGURE 3: Representative wave function amplitudes distributions corresponding to (a) an extended state, (b) an exponentially localized state, and (c) a critical state (adapted from [8] with permission from Ostlund and Pandit (c) 1984 by the American Physical Society).

situation is referred to as a passage through a "critical point". At the critical temperature various thermodynamic functions develop a singular behavior which is related to long-range correlations and large fluctuations. Actually, the system should appear identical on all length scales at, exactly, the critical temperature and, consequently, it would be scale invariant. All these features, characteristic of thermodynamic phase transitions, have been progressively incorporated to the study of both incommensurate and QPS, since analogous transitions can occur in a solid that preserves its structural integrity but experiences a transition from a metallic-like behavior to an insulator-like one, for instance. This kind of phase transitions, affecting the transport properties rather than the lattice structure of a given material, is very important to us, since the metal-insulator transition provides the basic grounds necessary to introduce some fundamental notions and the related terminology.

Indeed, the metallic regime is understood in terms of conducting extended electronic wave functions propagating through the solid (Figure 3(a)), whereas the insulating regime is explained in terms of decaying wave functions corresponding to states localized close to the lattice atoms (Figure 3(b)). During the metal-insulator transition the electronic wave functions experience substantial changes, exhibiting a rather 


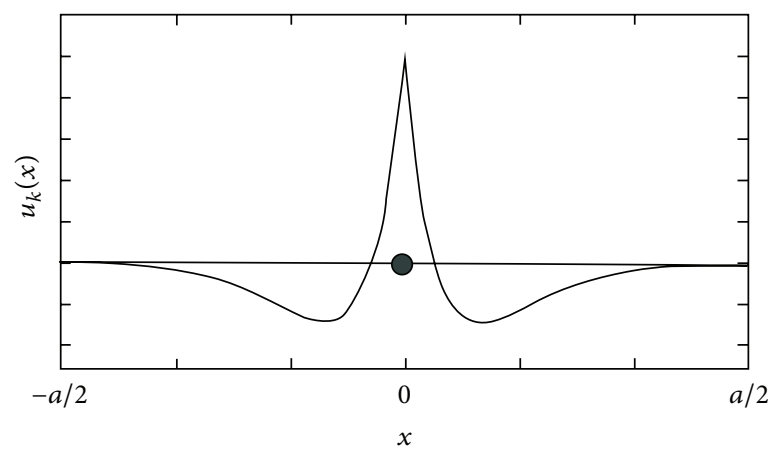

(a)

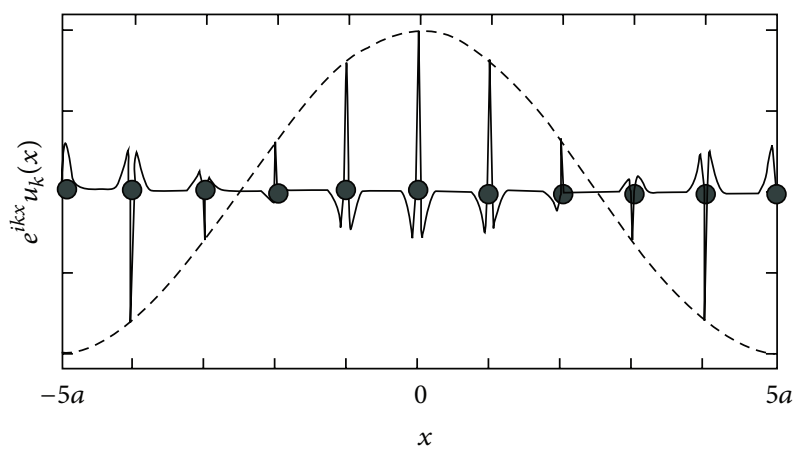

(b)

FIGURE 4: Illustration of a Bloch function. In (a) we show the periodic function $u_{k}(x)$ centered at the origin of the unit cell within the range $-a / 2 \leq x \leq a / 2$, where $a$ is the lattice constant. In (b) the Bloch function is constructed by using the function shown in (a). At every lattice site (solid circles representing atoms) the function $u_{k}$ is modulated by the plane wave $e^{i k x}$ (only the real part is plotted) (Courtesy of Uichiro Mizutani from [9] by permission of Cambridge University Press).

involved oscillatory behavior and displaying strong spatial fluctuations at different scales (Figure 3(c)). Due to this peculiar spatial distribution of their amplitudes (reminiscent of the atomic distribution observed in materials undergoing a structural phase transition at the critical point) these electronic states are referred to as critical wave functions.

In order to properly appreciate the main characteristic features of critical states, let us recall first the explicit mathematical expressions for extended and localized states. It is well known that in periodic crystals extended states are described in terms of the so-called Bloch functions. The conceptual appeal of Bloch functions in the description of the physical properties of classical crystals is easily grasped by solving the Schrödinger equation describing the motion of an electron with a wave function $\psi$, energy $E$, and effective mass $m$, under the action of a potential $V(x)$ in one dimension:

$$
\frac{\hbar^{2}}{2 m} \frac{d^{2} \psi}{d x^{2}}+[E-V(x)] \psi=0,
$$

where $\hbar$ is the reduced Planck's constant. In the absence of any interaction (i.e., $V(x)=0$, for all $x$ ) the solution to (5) for a free electron is readily obtained as a linear combination of plane waves of the form $\psi_{ \pm}(x)=A_{ \pm} e^{ \pm i k x}$, where $k=\sqrt{2 m E} / \hbar$ is the wave vector. The next step is to consider the motion of an electron interacting with the atoms forming a crystal lattice with a lattice constant $a$. Since this lattice is periodic in a classical crystal, the resulting interaction potential naturally inherits the periodicity of the lattice, so that one has $V(x+$ $n a)=V(x)$, where $n \in \mathbb{Z}$. Within this context, the celebrated Bloch's theorem states that the solution to (5) now reads

$$
\psi(x)=u_{k}(x) e^{i k x},
$$

where the function $u_{k}(x)$ is real and periodic, with the same period than that of the lattice; that is, $u_{k}(x+n a)=u_{k}(x)$, for all $n \in \mathbb{Z}$. In addition, the $u_{k}(x)$ function generally depends on the electron wave vector, which can take certain values comprised within a series of allowed intervals, $-m \pi / a \leq$ $k \leq m \pi / a, m \in \mathbb{N}$, which ultimately define the electronic energy spectrum. Therefore, the periodicity of function $u_{k}(x)$ guarantees the periodicity of the Bloch function itself, for

$$
\begin{aligned}
\psi(x+n a) & =u_{k}(x+n a) e^{i k(x+n a)} \\
& =u_{k}(x) e^{i k x} e^{i k n a}=u_{k}(x) e^{i k x}=\psi(x) .
\end{aligned}
$$

It is important to note that the $u_{k}(x)$ function usually describes the structure of the wave function in the atoms neighborhood, and it is generally relatively localized around them (Figure 4(a)). Thus, the extended nature of Bloch functions ultimately arises from the plane wave modulation, as it is illustrated in Figure 4(b).

On the other hand, in amorphous materials characterized by a random distribution of atoms through the space, the electronic states are exponentially localized according to an expression of the form

$$
\psi_{n}(x)=A_{n} e^{-\ell_{n}|x|},
$$

where $n$ labels the lattice position and $\ell_{n}^{-1}$ provides a measure of the spatial extension of the wave function, which is referred to as its localization length. At this point it is important to emphasize that the ultimate reason leading to the localization of electronic states in random chains is not the presence of exponentially decaying modulations in (8) but the fact that both the amplitudes $A_{n}$ and the reciprocal localization lengths $\ell_{n}$ form a random ensemble [10]. This property guarantees that possible resonances between electronic states belonging to neighboring atoms cannot extend to other atoms located far away along the chain. In fact, as soon as a short range correlation is present in an otherwise disordered chain, one can observe the emergence of a significant number of relatively extended states $[11,12]$.

In summary, Bloch states are the prototypical states of periodic systems, whereas exponentially localized states are the typical states found in random systems. ${ }^{3}$ Accordingly, the states occurring at the critical point in a metal-insulator transition, that is, critical states, were originally defined as being neither Bloch functions nor exponentially localized 
states but occupying a fuzzy intermediate position between them.

These states, which we will term critical, have a maximum at a site (in the lattice) and a series of subsidiary maxima at (a number of other) sites which do not decay to zero [13].

As we previously mentioned, the term "critical" was originally borrowed from thermodynamics, where it has usually been applied to describe a conventional phase transition where a state undergoes fluctuations in certain physical properties which are the same on all length scales. Following a chronological order the concept of critical wave function was born in the study of the Anderson Hamiltonian which describes a regular lattice with site-diagonal disorder. This model is known to have extended states for weak disorder in $3 \mathrm{D}$ systems, as well as in $2 \mathrm{D}$ samples with a strong magnetic field. For strong disorder, on the other hand, the electronic states are localized. For 1D systems it was proved that localized states decay exponentially in space in most cases [14]. However, this exponential decay relates to the asymptotic evolution of the envelope of the wave function, while the short-range behavior is characterized by strong fluctuations. The magnitude of these fluctuations seems to be related to certain physical parameters, such as the degree of disorder which, in turn, controls the appearance of the socalled mobility edges. Approaching a mobility edge, from the insulator regime, the exponential decay constant diverges so that the wave function amplitudes can be expected to feature fluctuations on all length scales larger than the lattice spacing. This singular fact turns out to be very convenient to explain metal-insulator transitions.

Thus, the notion of "criticality" can be understood as follows. An extended wave function is expected to extend homogeneously over the whole sample. On the other hand, for a wave function localized at a particular site of the sample, one expects its probability density to display a single dominant maximum at, or around, this site, and its envelope function is generally observed to decay exponentially in space. On the contrary, a critical state is characterized by strong spatial fluctuations of the wave function amplitudes. This unusual behavior, consisting of an alternatively decaying and recovering of the wave function amplitudes, is illustrated in Figure 5. Two main features of this wave function amplitude distribution must be highlighted. On the one hand, although the main local maxima are modulated by an overall decaying envelope, this envelope cannot be fitted to an exponential function. On the other hand, the subsidiary peaks around the main local maxima display self-similar scaling features.

1.3. Incommensurate and Self-Similar Systems. The discovery of QCs spurred the interest in the study of specific QP lattice models describing the electron dynamics in one-dimensional QPS. As a first step, most studies made use of both the electron independent and the tight-binding approximations, ${ }^{4}$ by considering a discretized version of the time-independent Schrödinger equation (5) given by

$$
t_{n, n+1} \psi_{n+1}+t_{n, n-1} \psi_{n-1}=\left(E-V_{n}\right) \psi_{n},
$$

TABLE 1: Functions determining the potential energy values $V_{n}$ in (9) for different incommensurate models considered in the literature, where $\lambda>0$ is the amplitude of the potential, $v$ is an arbitrary phase, $x(n) \equiv n+\sigma[n \sigma], \sigma=(\sqrt{5}-1) / 2$ is the inverse of the golden mean, and $b$ is a real number.

\begin{tabular}{lc}
\hline Model & Potential \\
\hline Aubry and André & $\lambda \cos (n \alpha+\nu)$ \\
Bichromatic & $\lambda_{1} \cos (n+\nu)+\lambda_{2} \cos (n \alpha+\nu)$ \\
Soukoulis and Economou & $\lambda \cos (n \alpha+\nu)+\lambda^{\prime} \cos 2(n \alpha+\nu)$ \\
Maryland & $\lambda \tan (n \alpha+\nu)$ \\
Kim & $\lambda \cos (x(n) \alpha+\nu)$ \\
Hiramoto & $\lambda \tanh [b \cos (n \alpha+\nu)] / \tanh b$ \\
\hline
\end{tabular}

where $\psi_{n}$ stands for the amplitude of the wave function in the $n$th lattice site of the chain, $V_{n}$ are the on-site energies (accounting for the atomic potentials) at that site, $t_{n, n \pm 1}$ are the corresponding transfer integrals (accounting for the hopping of the electron between neighboring atoms), and $E$ is the energy of the state. ${ }^{5}$ In the first place we note that this equation reduces to well-known systems of physical interest in certain particular cases. For instance, if $V_{n}$ and/or $t_{n, n \pm 1}$ are uncorrelated random variables with uniform probability distribution, (9) describes a disordered system within the so-called Anderson model. On the contrary, if the $V_{n}$ and $t_{n, n \pm 1}$ parameters obey a periodic sequence, we will be dealing with a classical periodic crystal. Therefore, (9) allows for a unified mathematical treatment encompassing periodic, random, and QPS.

In order to specify a given QPS one must indicate its onsite energies $\left\{V_{n}\right\}$ and transfer integrals $\left\{t_{n, n+1}\right\}$ sequences. Potentials usually considered in (9) can be classified into two broad families, namely, incommensurate and self-similar potentials.

(i) Incommensurate potentials are characterized by the presence of (at least) two superimposed periodic structures whose corresponding periods are incommensurate. The origin of incommensurability may be structural (as it occurs when two different periodic sublattices form a whole system) or dynamical, when one of the periodicities is associated with the crystal structure and the other one is related to the behavior of elementary excitations propagating through the solid (see Section 1.5).

(ii) Self-similar potentials, on the contrary, describe a new ordering of matter based on the presence of inflation symmetries replacing the translation ones.

In the case of incommensurate systems, the potential amplitude is given by a periodic function whose argument depends on $n \alpha, \alpha$ being an irrational number. Some of the most representative models studied up to now are given in Table 1.

On the other side, in most self-similar systems studied to date the aperiodic sequence $V_{n}$ is defined in terms of certain substitutional sequences. A substitution sequence is formally defined by its action on an alphabet $\mathscr{A}=\{A, B, C, \ldots\}$, which consists of certain number of letters, generally corresponding 


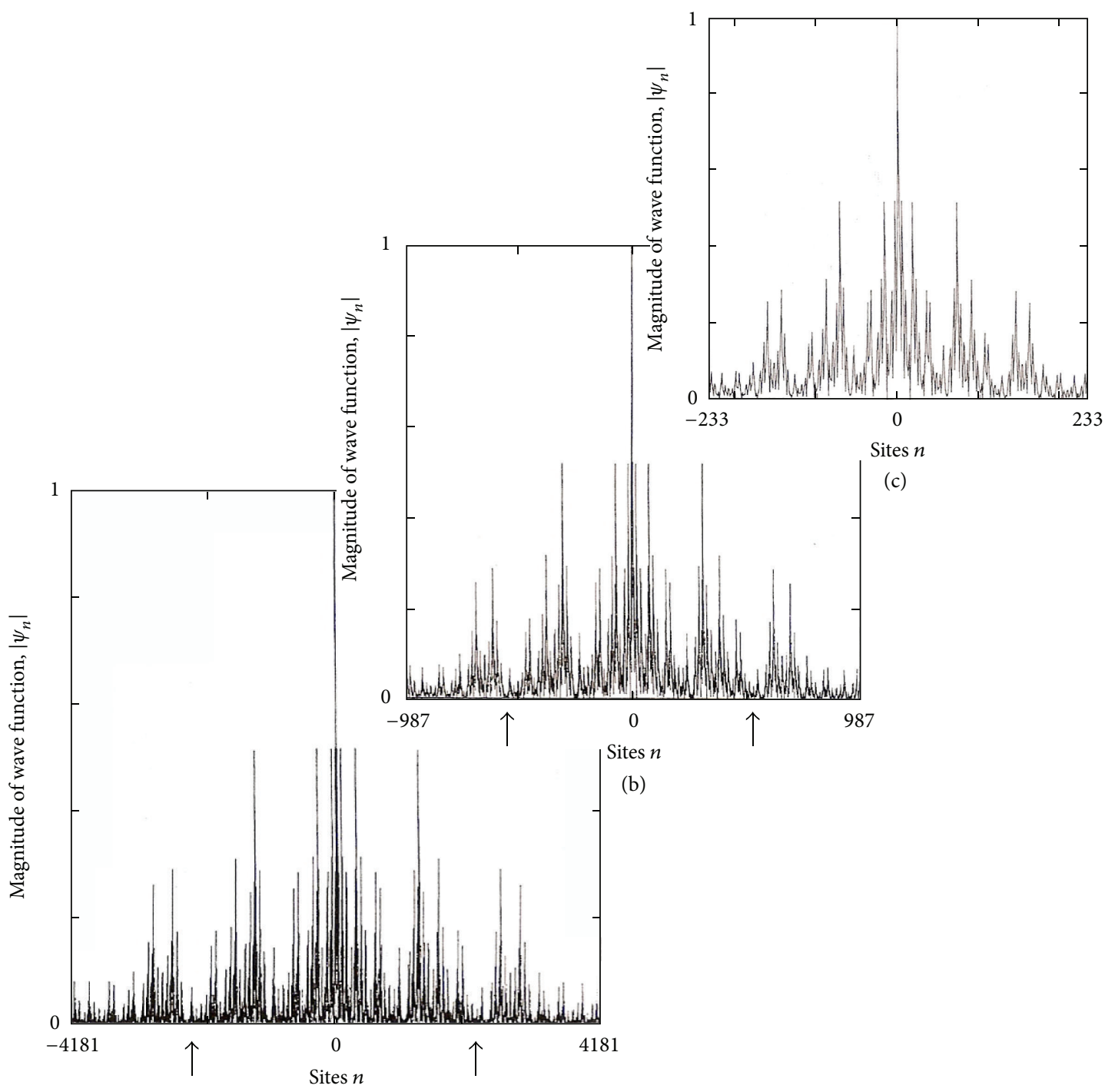

(a)

FIGURE 5: Illustration of a critical wave function. The amplitude distribution shown in (a) for the wave function as a whole is identically reproduced at progressively smaller scales (indicated by the arrows) in (b) and (c) after a proper rescaling. This is a distinctive feature of its characteristic self-similarity property. This wave function corresponds to the eigenstate located at the center of the spectrum, $E=0$, in the Aubry-André model (see Section 1.5) with a potential strength $\lambda=2$ (adapted from [8], with permission from Ostlund and Pandit (c) 1984 by the American Physical Society).

to different kinds of atoms in actual realizations. The substitution rule starts by replacing each letter by a finite word, as it is illustrated in Table 2. The corresponding aperiodic sequence is then obtained by iterating the substitution rule $g$ starting from a given letter of the set $\mathscr{A}$ in order to obtain a QP string of letters.

For instance, the Fibonacci sequence is obtained from the continued process $A \rightarrow A B \rightarrow A B A \rightarrow A B A A B \rightarrow$ $A B A A B A B A \rightarrow \cdots$. Another popular sequence is the so-called Thue-Morse sequence, which has been extensively studied in the mathematical literature as the prototype of a sequence generated by substitution. In this case the continued process reads $A \rightarrow A B \rightarrow A B B A \rightarrow A B B A B A A B \rightarrow$ $A B B A B A A B B A A B A B B A \rightarrow \cdots$. The number of letters in this sequence increases geometrically, $N=2^{k}$, where $k$ indicates the iteration order. In the infinite limit the relative frequency of both kinds of letters in the sequence takes the same value; that is, $v_{A}=v_{B}=1 / 2$. This result contrasts with that corresponding to the Fibonacci sequence, where $v_{A}=\tau^{-1}$ and $v_{B}=\tau^{-2}$, with $\tau=(1+\sqrt{5}) / 2$ being the golden mean. Another important difference is that in the Fibonacci sequence $B$ letters always appear isolated, whereas in ThueMorse sequence both dimers $A A$ and $B B$ appear alike.

We note that QPS based on substitution sequence related alphabets take on just a few possible values (say two for Fibonacci and Thue-Morse or four for Rudin-Shapiro sequences), whereas QPS based on the discretization of continuous potentials (e.g., $V_{n}=\lambda \cos (n \alpha+\nu)$ ) can take on 
TABLE 2: Substitution rules most widely considered in the study of self-similar systems, where $n$ and $m$ are positive integers.

\begin{tabular}{|c|c|c|}
\hline Sequence & Set $\mathscr{A}$ & Substitution rule \\
\hline Fibonacci & $\{A, B\}$ & $\begin{array}{c}g(A)=A B \\
g(B)=A\end{array}$ \\
\hline Silver mean & $\{A, B\}$ & $\begin{array}{c}g(A)=A A B \\
g(B)=A\end{array}$ \\
\hline Bronze mean & $\{A, B\}$ & $\begin{array}{c}g(A)=A A A B \\
g(B)=A\end{array}$ \\
\hline Precious means & $\{A, B\}$ & $\begin{array}{c}g(A)=A^{n} B \\
g(B)=A\end{array}$ \\
\hline Copper mean & $\{A, B\}$ & $\begin{array}{c}g(A)=A B B \\
g(B)=A\end{array}$ \\
\hline Nickel mean & $\{A, B\}$ & $\begin{array}{c}g(A)=A B B B \\
g(B)=A\end{array}$ \\
\hline Metallic means & $\{A, B\}$ & $\begin{array}{c}g(A)=A B^{n} \\
g(B)=A\end{array}$ \\
\hline Mixed means & $\{A, B\}$ & $\begin{array}{c}g(A)=A^{n} B^{m} \\
g(B)=A\end{array}$ \\
\hline Thue-Morse & $\{A, B\}$ & $\begin{array}{r}g(A)=A B \\
g(B)=B A\end{array}$ \\
\hline Period-doubling & $\{A, B\}$ & $\begin{array}{l}g(A)=A B \\
g(B)=A A\end{array}$ \\
\hline Ternary Fibonacci & $\{A, B, C\}$ & $\begin{array}{c}g(A)=A C \\
g(B)=A \\
g(C)=B\end{array}$ \\
\hline Rudin-Shapiro & $\{A, B, C, D\}$ & $\begin{array}{l}g(A)=A B \\
g(B)=A C \\
g(C)=D B \\
g(D)=D C\end{array}$ \\
\hline Paper folding & $\{A, B, C, D\}$ & $\begin{array}{l}g(A)=A B \\
g(B)=C B \\
g(C)=A D \\
g(D)=C D\end{array}$ \\
\hline
\end{tabular}

a significantly larger set of values. This property makes these potentials more complex from Shanon's entropy viewpoint [15].

1.4. Spectral Measure Classification. A key question in any general theory of QPS regards the relationship between their atomic topological order, determined by a given $\mathrm{QP}$ distribution of atoms and bonds, and the physical properties stemming from that structure. At the time being a general theory describing such a relationship is still lacking. This unsatisfactory situation has considerably spurred the interest in studying the main properties of QP Schrödinger operators from a mathematical perspective. To this end, it is convenient to arrange (5) in the following form ${ }^{6}$ :

$$
\left[-\frac{d^{2}}{d x^{2}}+V(x)\right] \psi=E \psi
$$

which can be regarded as an eigenvalue equation involving the Schrödinger operator within the square bracket. Within this framework the nature of an (eigen)state is determined by the measure of the spectrum to which it belongs.
Most rigorous mathematical results in the field have been derived from the study of nearest-neighbor, tight-binding models described in terms of a convenient discretization of (10) given by the Hamiltonian ${ }^{7}$ :

$$
(H \psi)_{n}=t \psi_{n+1}+t \psi_{n-1}+\lambda \vartheta(n) \psi_{n}, \quad n \in \mathbb{Z},
$$

where $\lambda>0$ measures the potential strength, $\vartheta(n)=[(n+$ $1) \alpha]-[n \alpha]$, where $[x]$ denotes the integer part of $x$, and $\alpha$ is generally the golden mean or its reciprocal. From a mathematical point of view these models belong to the class of AP Schrödinger operators, which display unusual spectral properties.

Indeed, according to Lebesgue's decomposition theorem, the energy spectrum of any measure in $\mathbb{R}^{n}$ can be uniquely decomposed in terms of just three kinds of spectral measures (and mixtures of them), namely, pure-point $\left(\mu_{P}\right)$, absolutely continuous $\left(\mu_{\mathrm{AC}}\right)$, and singularly continuous $\left(\mu_{\mathrm{SC}}\right)$ spectra, in the following form:

$$
\mu=\mu_{P} \cup \mu_{\mathrm{AC}} \cup \mu_{\mathrm{SC}} .
$$

Suitable examples of physical systems containing both the pure-point and/or the absolutely continuous components in their energy spectra are well known, with the hydrogenic atom being a paradigmatic instance. On the other hand, the absence of actual physical systems exhibiting the singular continuous component relegated this measure as a merely mathematical issue for some time. From this perspective, the discovery of QCs bridged the long standing gap between the theory of spectral operators in Hilbert spaces and condensed matter theory $[16,17]$.

Now, from the viewpoint of condensed matter physics there are two different (though closely related) measures one can consider when studying the properties of solid materials. On the one hand, we have the measure related to the atomic density distribution, which determines the spatial structure of the solid. On the other hand, we have the measure related to the energy spectra of the system, which describes the electronic structure (or the frequency distribution of atomic vibrations in the case of the phonon spectrum) and its related physical properties. In order to characterize the solid structure it is convenient to focus on the nature of the measure associated with the lattice Fourier transform, which is related to the main features of X-ray, electron, or neutron diffraction patterns. For the sake of illustration in Figure 6 we show the Fourier amplitude distributions for three representative QPS [18].

Since the electronic structure of a system is ultimately related to the spatial distribution of its constituent atoms throughout the space and to their bonding properties, one expects a close relation to exist between the electronic energy spectral measure and the Fourier lattice measure. In this regard a particularly relevant result obtained from the study of QPS is the so-called gap-labeling theorem, which provides a relationship between reciprocal space (Fourier) spectra and Hamiltonian energy spectra. In fact, this theorem relates the position of a number of gaps in the electronic energy spectra to the singularities of the Fourier transform of the substrate lattice [19-21]. Accordingly, in order to gain additional insight 

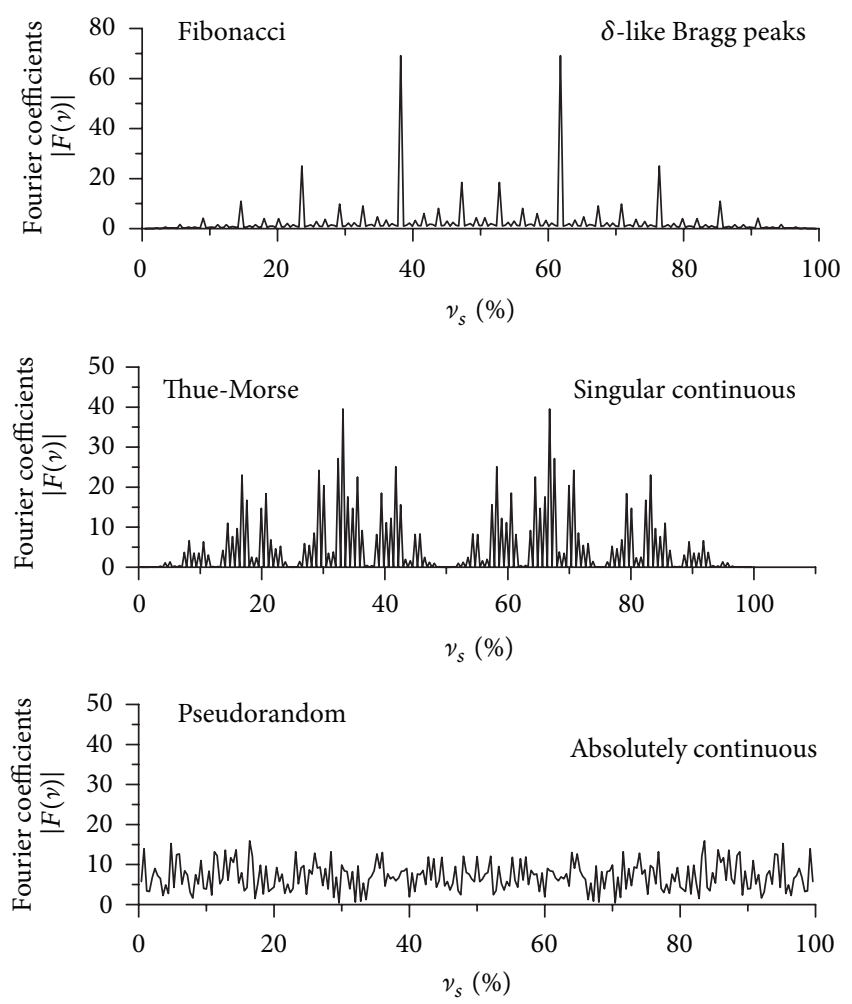

Figure 6: Absolute value of the Fourier coefficients of a quasiperiodic (Fibonacci) structure, an aperiodic Thue-Morse structure with a singular-continuous spectrum, and an aperiodic Rudin-Shapiro structure with an absolutely continuous spectrum (Courtesy of Luca Dal Negro).

into the relationship between the type of structural order present in an aperiodic solid (as determined by its lattice Fourier transform) and its related transport properties (as determined by the main features of the energy spectrum and the nature of its eigenstates) it is convenient to introduce the chart depicted in Figure 7. In this chart we present a classification scheme of aperiodic systems based on the nature of their diffraction spectra (in abscissas) and their energy spectra (in ordinates). In this way, we clearly see that the simple classification scheme based on the periodic-amorphous dichotomy is replaced by a much richer one, including nine different entries. In the upper left corner we have the usual periodic crystals exhibiting pure-point Fourier spectra (well-defined Bragg diffraction peaks) and an absolutely continuous energy spectrum (Bloch wave functions in allowed bands). In the lower right corner we have amorphous matter exhibiting an absolutely continuous Fourier spectrum (diffuse spectra) and a pure-point energy spectrum (exponentially localized wave functions). By inspecting this chart, one realizes that although Fibonacci and Thue-Morse lattices share the same kind of energy spectrum (a purely singular continuous one), they have different lattice Fourier transforms so that these QPS must be properly classified into separate categories.

At the time being, the nature of the energy spectrum corresponding to the Rudin-Shapiro lattice is yet an open question. Numerical studies suggested that some electronic

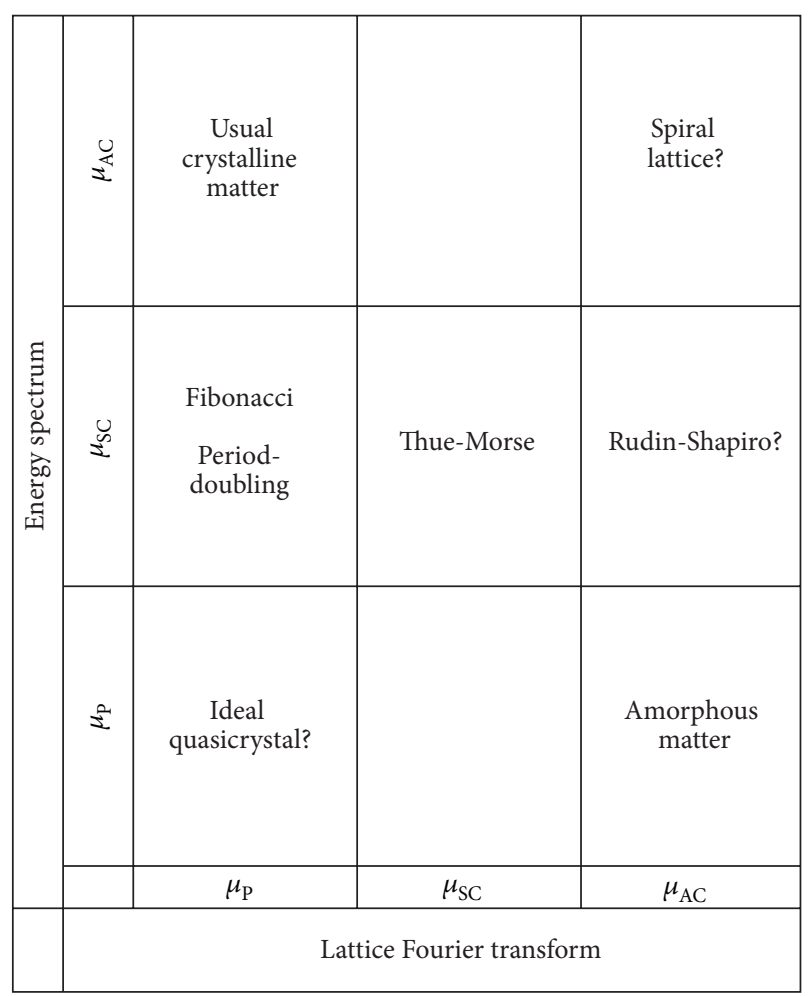

FIgURE 7: Classification of aperiodic systems attending to the spectral measures of their lattice Fourier transform and their Hamiltonian spectrum energy (from [22], Maciá, with permission from IOP Publishing Ltd.).

states are localized in these lattices, in such a way that the rate of spatial decay of the wave functions is intermediate between power and exponential laws [23-25]. These results clearly illustrate that there is not any simple relation between the spectral nature of the Hamiltonian describing the dynamics of elementary excitations propagating through an aperiodic lattice and the spatial structure of the lattice potential.

In the light of these results, it is tempting to establish a one-to-one correspondence between extended, localized and critical states introduced in Section 1.2, on the one hand, and the three possible spectral measures, namely, absolutely continuous, pure-point, and singular continuous spectra, on the other hand. Indeed, the study of several physical systems provided evidence on the correspondence between extended states and absolutely continuous spectra, as well as between exponentially localized states and pure-point measures. For instance, periodic lattices described in terms of the KronigPenney model have a mixed spectrum consisting of a purepoint component for low energy values and an absolutely continuous component for higher enough energies. ${ }^{8}$

What about singular continuous spectra? The energy spectrum of most QPS considered to date seems to be a purely singular continuous one, which is supported on a Cantor set of zero Lebesgue measure. Thus, the spectrum exhibits an infinity of gaps and the total bandwidth of the allowed states vanishes in the thermodynamic limit. Though this property has only been proven rigorously for QPS based 
on the Fibonacci [26-28], Thue-Morse, and period-doubling sequences $[29,30]$, it is generally assumed that it may be a quite common feature of most QPS [31], and it has become a relatively common practice to refer to their states generically as critical states on this basis. However, this semantics does not necessarily imply that all these critical states, which belong to quite different QPS in the spectral chart shown in Figure 7, will behave in exactly the same way from a physical viewpoint. This naturally leads to some misleading situations.

In order to clarify this issue one should start by addressing the following questions.

(i) What is the best term to refer to the eigenstates belonging to a singular continuous spectrum?

(ii) What are the specific features (if any) of these states as compared to those belonging to absolutely continuous or pure-point spectra?

(iii) What are the characteristic physical properties (if any) of states belonging to singular continuous spectra?

(iv) To what extent are these properties different from those exhibited by Bloch states and exponentially localized states, respectively?

To properly answer the above questions, one may reasonably expect that the study of a system where the three possible kinds of wave functions were simultaneously present may shed some light on the physical nature of critical wave functions and their main differences with respect to both Bloch and exponentially localized functions.

1.5. The Aubry-André Model. In 1980 Aubry and André predicted that for a certain class of one-dimensional QPS a metal-insulator localization phase transition can occur [32]. Below the transition all the states of the system are extended, whereas above the critical point all states are localized. At exactly the critical point all the wave functions become critical ones. Therefore, the Aubry-André model provides an illustrative example of a QPS which can exhibit extended, localized, or critical wave functions depending on the value of a control parameter which measures the potential strength. Accordingly, this parameter can be regarded as an order parameter controlling the existence of a metal-insulator transition in the system.

Explicitly, the Schrödinger equation for the Aubry-André model is given by

$$
\left[-\frac{d^{2}}{d x^{2}}+\lambda \cos (2 \pi \alpha+\nu)\right] \psi=E \psi
$$

where $\alpha$ is an irrational number, $\lambda$ is a real number modulating the potential strength, and $v$ is a real number describing a phase shift. There are two well-known physical systems which can be described by (13). The first is the motion of an electron in a two-dimensional square lattice with lattice constant $a$ in the presence of a magnetic field $\mathbf{H}$ perpendicular to the plane. In this case $\alpha=e a^{2} H /(h c)$ is related to the ratio of the magnetic flux through the lattice unit cell $\left(a^{2} H\right)$ to the quantum magnetic flux $(h c / e)$, where $c$ is the speed of light and $e$ is the electron charge. The second example is provided by the one-dimensional electron dynamics in an aperiodic crystal characterized by the presence of two superimposed periodic potentials: the main one, of period $L_{1}$, determines the position of the discrete lattice points, and the subsidiary one, of period $L_{2}$, describes a displacive modulation of the structure.

Within the tight-binding approach and the nearestneighbor approximation it is convenient to discretize (13) in the form: ${ }^{9}$

$$
\psi_{n-1}+\psi_{n+1}+[E-\lambda \cos (2 \pi n \alpha+\nu)] \psi_{n}=0,
$$

where $n$ labels the lattice sites and the lattice constant defines the length scale $(a \equiv 1)$. Considered as an operator in $\ell^{2}(\mathbb{Z}),(14)$ describes a bounded and self-adjoint operator. For a rational value of $\alpha$ (periodic crystal) (14) can be solved by applying Bloch's theorem and the energy spectrum is absolutely continuous. For irrational values of $\alpha$ (aperiodic crystal) the nature of the energy spectrum depends on the value of the potential strength $\lambda$. For $\lambda<2$ the spectrum is absolutely continuous with extended Bloch wave functions (Figure 8); when $\lambda>2$ the spectrum is pure-point and contains exponentially localized states. At $\lambda=2$ the spectrum is singular continuous and all the wave functions become critical (Figure 5). ${ }^{10}$

The fact that when the critical potential strength $\lambda=$ 2 is adopted, the metal-insulator transition occurs for all the eigenstates, independently of their energy, was rather surprising. Indeed, previous experience with random systems showed that the states closer to the band edges become more easily localized than those located at the band center. It is now understood that the simultaneous change of the localization degree for all the eigenstates is a unique property of the socalled self-dual systems, of which the Aubry-André model is a typical representative. The self-dual property expresses the following symmetry: if we substitute

$$
\psi_{n}=e^{i 2 \pi k n} \sum_{m=-\infty}^{\infty} a_{m} e^{i m(2 \pi n \alpha+\nu)}
$$

in (14) we obtain the so-called dual representation:

$$
a_{m+1}+a_{m-1}+[\widetilde{E}-\tilde{\lambda} \cos (2 \pi n \alpha+\widetilde{v})] a_{m}=0,
$$

where the dual variables are

$$
\tilde{\lambda}=\frac{4}{\lambda}, \quad \widetilde{E}=\frac{2 E}{\lambda}, \quad \widetilde{\nu}=k .
$$

By comparing (14) and (16) we see that the Fourier coefficients $a_{m}$ satisfy the same eigenvalue equation as the wave functions amplitudes $\psi_{n}$ when $\lambda=2$. In this case it is said that (14) and (16) are self-dual (remain invariant under a Fourier transformation). Accordingly, if the eigenstate $\psi_{n}$ is spatially localized, then the eigenstate of the dual problem, $a_{m}$, is spatially extended and vice versa.

The degree of localization of the Aubry-André model states can be collectively characterized by an exponent $\beta$, defined as [8]

$$
\beta \equiv \lim _{m \rightarrow \infty}\left[\frac{1}{p \ln \alpha} \ln \frac{S_{m}}{S_{m+p}}\right], \quad S_{m} \equiv \frac{1}{q_{m}} \sum_{n=-q_{m}}^{q_{m}}\left|\psi_{n}\right|^{2},
$$




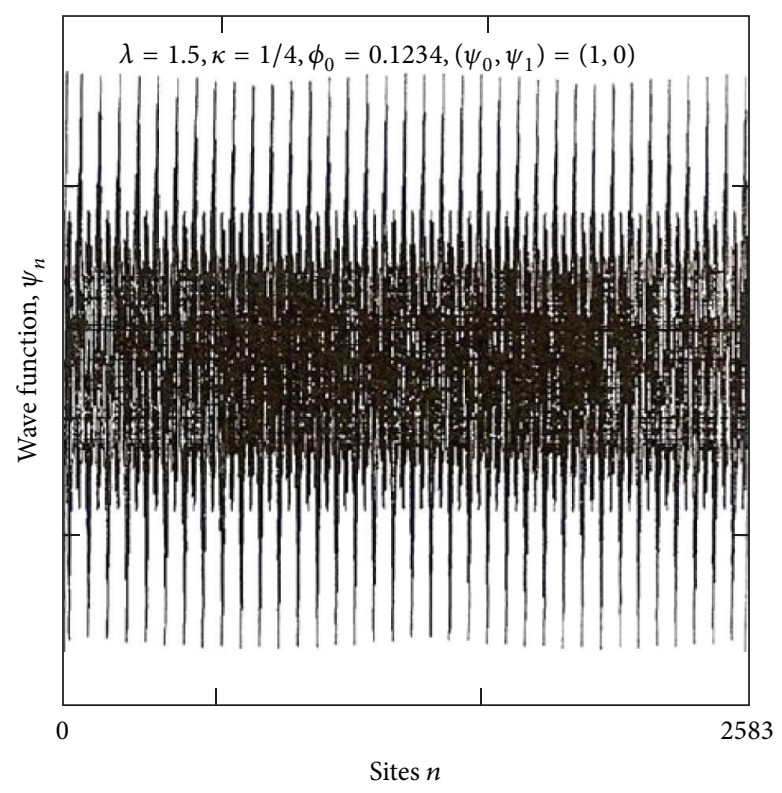

(a)

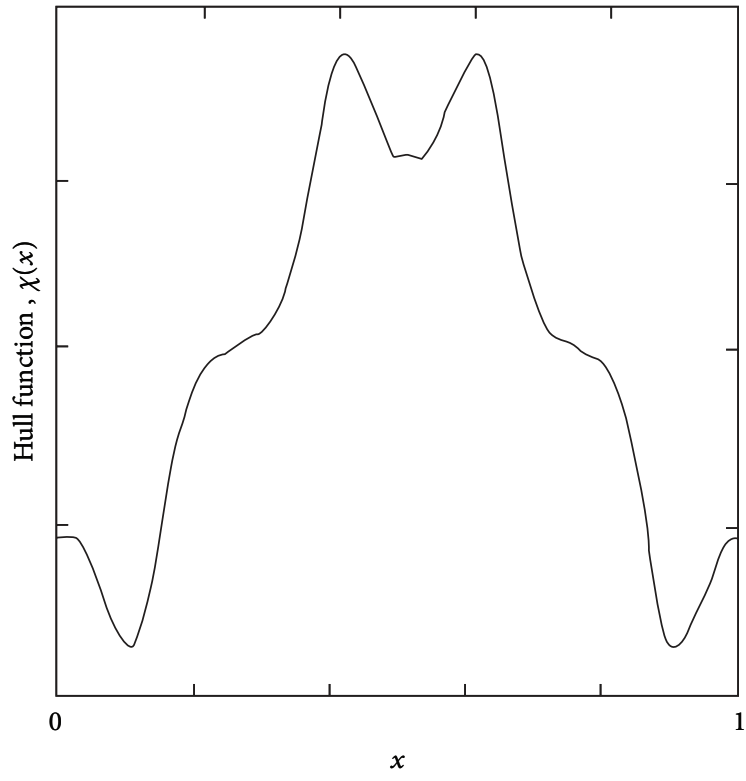

(b)

FIGURE 8: Illustration of a Bloch function in the weak potential regime of the Aubry-André model. The amplitude distribution shown in (a) for the wave function as a whole can be expressed in the form $\psi_{n}=e^{i n \pi / 2} u(x)$, where the hull periodic function $u(x)=u(x+1)$ is shown in (b). This wave function corresponds to the eigenstate located at the center of the spectrum, $E=0$, with a potential strength $\lambda=1.5$ (adapted from [8], with permission from Ostlund and Pandit (c) 1984 by the American Physical Society).

where $p$ is some integer (which may depend on $k$ ) describing the lattice scaling properties, $q_{m}$ is the denominator of successive rational approximants to $\alpha,{ }^{11}$ and $\left|\psi_{n}\right|^{2}$ is the squared wave function amplitude. From the above definition it follows that $\beta=0$ for extended states and $\beta=-1$ for exponentially decaying localized states. In their numerical study Östlund and Pandit considered the Aubry-André model with $\alpha=$ $\sigma=(\sqrt{5}-1) / 2$, the reciprocal of the golden mean, and they focused on the eigenstate corresponding to the energy $E=0$ (located at the spectrum center). By assuming $p=3$ (as suggested by the lattice scaling of the critical wave functions shown in Figure 5) they found

$$
\beta \equiv \begin{cases}0, & \lambda<2, \\ -(0.639 \pm 0.005), & \lambda=2, \\ -1, & \lambda>2 .\end{cases}
$$

Thus, one obtains Bloch functions when $\lambda<2$ (Figure 8) and exponentially localized functions when $\lambda>2$, whose localization length depends on the adopted potential strength according to the relationship $\ell^{-1} \sim|\lambda-2|$ (Figure 9). The value of the exponent $\beta$ obtained for $\lambda=2$ clearly indicates the states are neither exponentially localized nor Bloch-like extended, since $-1<\beta<0$ in this case. From a closer inspection of the wave function amplitudes distribution plotted in Figure 5 they concluded that the structure around the main local peaks approaches a length-rescaled version of the structure around $n=0$ peak. Let $n_{k}$ be a label denoting the location of the subsidiary peaks around the $n$th main local peak; then their corresponding amplitudes are related to $\psi_{n_{k}+n}=\varsigma \psi_{n}$, where $\varsigma=0.315 \pm 0.005$ is a scaling factor given by a constant fraction of the $n=0$ peak. Therefore, there is a whole hierarchy of subsidiary peaks with peak height $\varsigma^{m}$ of the central $n=0$ peak. The self-similar behavior of the critical wave function implies that, strictly speaking, they cannot be regarded as localized, since the wave function amplitudes never decay to zero, although the points exhibiting large amplitudes are further and further apart from each other.

It is important to highlight that the self-similar distribution of the wave function amplitudes is not restricted to the critical potential strength value $\lambda=2$. In Figure 9 the magnitude of the wave function at the band center is shown at the supercritical regime $\lambda=2.05$. By comparing Figures 5 and 9 we see that for potential strengths relatively close to the critical one, but certainly located in the supercritical regime, the wave functions envelope decays more rapidly, hence leading to a more pronounced confinement of the wave function support around the central site $n=0$, as expected for eigenstates undergoing a transition to an exponentially localized behavior. But this envelope is still encapsulating a self-similar amplitudes pattern distribution.

It was early suggested by Aoki that critical wave functions in the Aubry-André model may be characterized by some fractal dimensionality $[33,34]$. Later on Soukoulis and Economou [35] numerically demonstrated the fractal character of certain eigenfunctions in disordered systems and characterized their amplitude behavior by a fractal dimensionality. What is more interesting is that the fractal character of the wave function itself was suggested as a new method for finding mobility edges. The observation of anomalous scaling of both the moments of the probability distribution and the participation ratio near the localization 


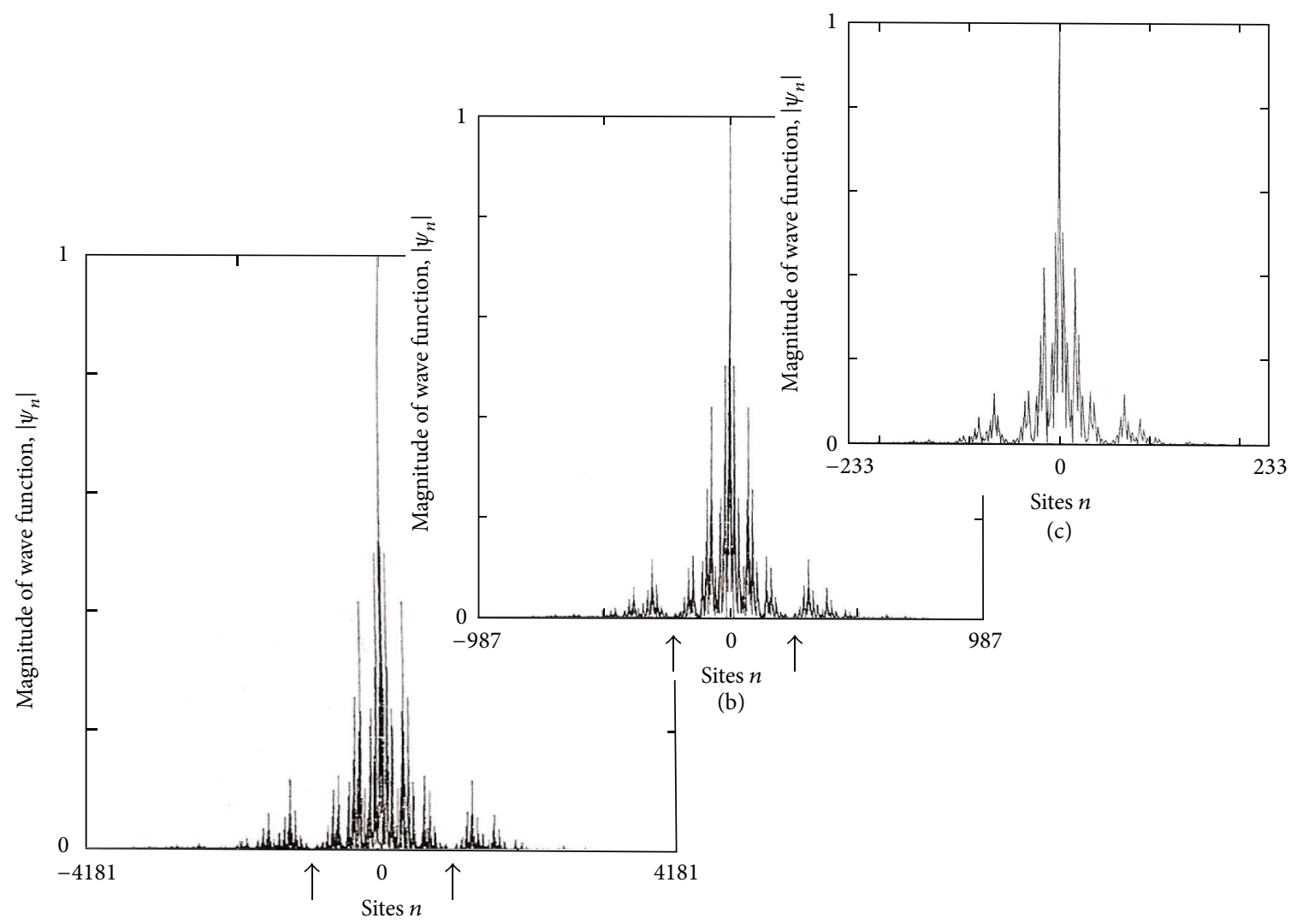

(a)

FIGURE 9: Illustration of a localized critical wave function. The amplitude distribution shown in (a) for the wave function as a whole is identically reproduced at progressively smaller scales in (b) and (c) after a proper rescaling. This is a distinctive feature of its characteristic self-similarity property. (This wave function corresponds to the eigenstate located at the center of the spectrum, $E=0$, in the Aubry-André model with a potential strength $\lambda=2.05$ ) (adapted from [8], with permission from Ostlund and Pandit (c) 1984 by the American Physical Society).

threshold in the Anderson model strongly suggested that a critical wave function cannot be adequately treated as simply fractal [36]. Instead, a multifractal measure is characterized by a continuous set of scaling indices $a$ and fractal dimensions $f(a)$. Accordingly, the wave functions cannot be described as homogeneous fractals [35, 37-40]. For an extended wave function one can obtain a single point $f=a=1$, which expresses the absence of self-similar features in the wave function amplitudes distribution. When a wave function is localized the $f(a)$ spectrum consists of two points (if the chain length is longer than the localization length), one being $f(0)=0$ and the other being $f(\infty)=1$. For a critical wave function one gets a continuous $f(a)$ spectrum, but a non-self-similar wave function shows quite different shapes in each scale and does not yield a $f(a)$ spectrum independent of the systems size. Thus, the self-similarity of a critical wave function is characterized by the size independence of its $f(a)$ spectrum.

1.6. Fractal Energy Spectra. In his pioneering article, Hofstadter put forward the following fundamental question: what is the meaning (if any) of a physical magnitude whose very existence depends on the rational or irrational nature of the numbers in terms of which this magnitude is expressed?

$$
\begin{aligned}
& \text { Common sense tells us that there can be no physi- } \\
& \text { cal effect stemming from the irrationality of some } \\
& \text { parameter, because an arbitrarily small change in } \\
& \text { that parameter would make it rational-and this } \\
& \text { would create some physical effect with the prop- } \\
& \text { erty of being everywhere discontinuous, which is } \\
& \text { unreasonable [41]. }
\end{aligned}
$$

To further analyze this question, in his study of the Harper's equation, ${ }^{12}$

$$
\psi_{n-1}+\psi_{n+1}+[E-2 \cos (2 \pi n \alpha+\nu)] \psi_{n}=0,
$$

he considered the dependence of the spectrum Lebesgue measure (see Section 1.3) as a function of the $\alpha$ parameter value and concluded that the measure has a very peculiar behavior: at rational values of $\alpha$ the measure is discontinuous, ${ }^{13}$ since there are irrationals arbitrarily near any rational, yet at irrational $\alpha$ values, the measure is continuous. This property is ultimately related to the highly fragmented nature of the energy spectrum for irrational $\alpha$ 


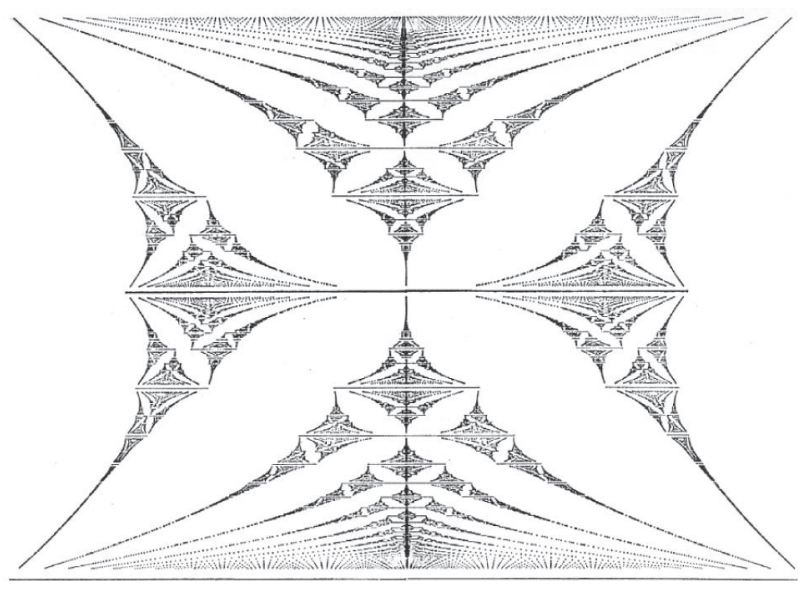

FIGURE 10: Harper's model energy spectrum for irrational $\alpha$ values. The energy is in abscissas, ranging between $E= \pm 4$. The fractional part of $\alpha$, ranging from 0 to 1 , is plotted in the ordinate axis (from [41], with permission from Hofstadter (C) 1976 by the American Physical Society).

values observed in his celebrated butterfly spectra shown in Figure 10.

\section{This graph has some very unusual properties. The large gaps form a very striking pattern somewhat resembling a butterfly; perhaps equally striking are the delicacy and beauty of the fine-grained structure. These are due to a very intricate scheme, by which bands cluster into groups, which them- selves may cluster into larger groups, and so on $[41]^{14}$}

Very similar features have been reported by different authors from the study of different QPS energy spectra (see Section 2.1) and can be summarized as follows.

(i) The energy spectrum of most self-similar systems exhibits an infinity of gaps and the total bandwidth of the allowed states vanishes in the $N \rightarrow \infty$ limit. This has been proven rigorously for systems based on the Fibonacci [27, 28], Thue-Morse, and period-doubling sequences [27].

(ii) The position of the gaps can be precisely determined through the gap labeling theorem in some definite countable set of numbers [19-21].

(iii) Scaling properties of the energy spectrum can be described using the formalism of multifractal geometry $[42,43]$.

An illustrative example of the spectrum structure corresponding to two QPS is shown in Figures 11 and 12. By inspecting these figures we clearly appreciate the following prefractal signatures:

(i) the spectra exhibit a highly fragmented structure generally constituted by as many fragments as the number of atoms present in the chain; (ii) the energy levels appear in subbands which concentrate a high number of states and which are separated by relatively wide forbidden intervals;

(iii) the degree of internal structure inside each subband depends on the total length of the chain, and the longer the chain, the finer the structure, which displays distinctive features of a self-similar distribution of levels.

Taken altogether these features provide compelling evidence about the intrinsic fractal nature of the numerically obtained spectra, which will eventually show up with mathematical accuracy in the thermodynamic limit $N \rightarrow \infty$. Now, as one approaches this limit it is legitimate to question whether a given energy value actually belongs to the energy spectrum. Indeed, this is not a trivial issue in the case of highly fragmented spectra supported by a Cantor set of zero Lebesgue measure and can be only guaranteed on the basis of exact analytical results. In fact, because of the presence of extremely narrow bands, special care is required in order to avoid studying states belonging to a gap and erroneously interpreting their features as those proper critical states.

In this regard, it should also be noted that irrational numbers cannot be explicitly included in a computing code as that but only in terms of approximate truncated decimal expressions. Accordingly, one must very carefully check that the obtained results are not appreciably affected by the truncation. To this end, one should consider the systematic use of successive approximants of an irrational number in order to explore the possible influence of its irrational character (if any) in the physical model under study. In fact, one can implement numerically an empirical scaling analysis in which the QPS is approximated by a sequence of periodic systems with progressively larger unit cells of size $q_{n}$ defined by the optimal rational approximants to $\alpha$; namely, $\alpha_{n}=p_{n} / q_{n}$. In this way, by checking that finer discretization produces almost the same results one can be confident enough of the reliability of the obtained results [44].

\section{One-Dimensional Aperiodic Systems}

Broadly speaking, an obvious motivation for the recourse to one-dimensional (1D) models in solid state physics is the complexity of the full-fledged problem. In the particular case of quasicrystalline matter this general motivation is further strengthened by the lack of translational symmetry, though the presence of a well-defined long-range orientational order in the system also prevents a naive application of procedures specifically developed for the study of random structures in this case.

We can also invoke more fundamental reasons supporting the use of $1 \mathrm{D}$ models as a first approximation to the study of realistic QPS. In fact, most characteristic features of QPS, like the fractal structures of their energy spectra and related eigenstates, can be explained in terms of resonant coupling effects in the light of Conway's theorem. Therefore, the physical mechanisms at work are not so dependent on the dimension of the system but are mainly determined by the self-similarity of the underlying structure [46]. Consequently, 


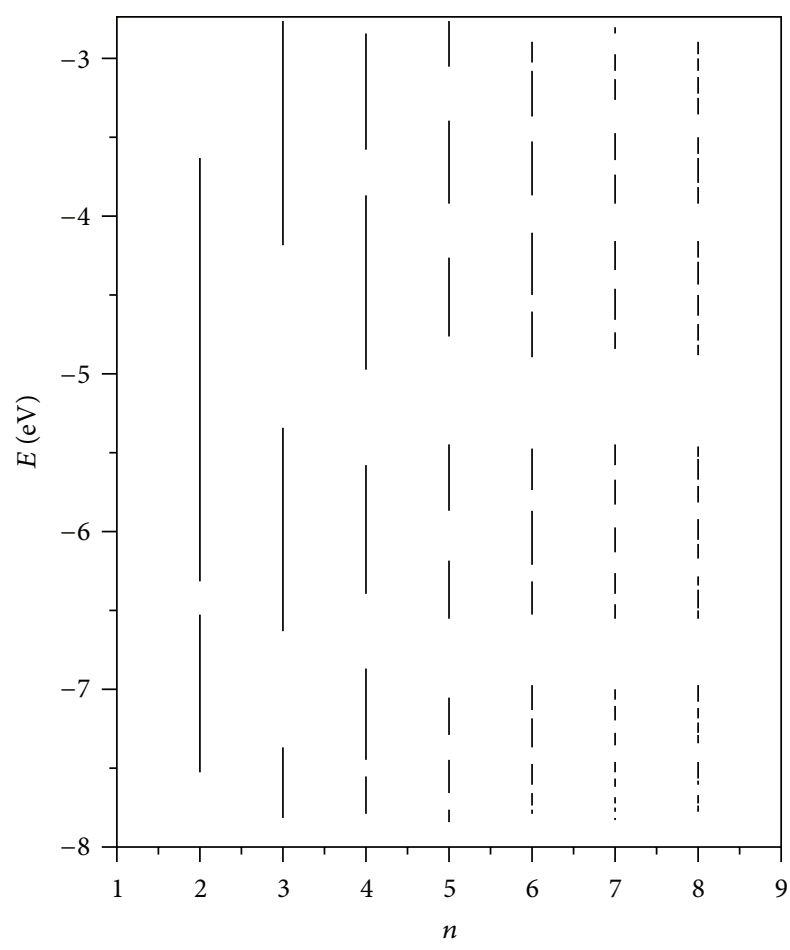

FIGURE 11: Fragmentation pattern of the energy spectrum of a transpolyacetylene quasiperiodic chain. The number of allowed subbands increases as a function of the system size expressed in terms of the Fibonacci order $n$ as $N=F_{n}$ (from [45], with permission from Elsevier).

the recourse to $1 \mathrm{D}$ models can be considered as a promising starting point, for such models encompass, in the simplest possible manner, most of the novel physics attributable to the QP order.

2.1. Quasiperiodic Binary Alloys. Several representative examples of binary Fibonacci chains composed of two types of atoms, say A and B, which have been profusely studied in the literature, are displayed in Figure $13 .{ }^{15}$ In these models the QP order can be introduced in

(i) the sequence of atomic potentials $\left\{V_{n}\right\}$ (Figure 13(b)); this arrangement is referred to as the on-site (or diagonal) model, and all the transfer integrals are assumed to be equal over the lattice $\left(t_{n, n+1}=t, \forall n\right)$ so that (9) reduces to

$$
\psi_{n+1}+\psi_{n-1}=\left(E-V_{n}\right) \psi_{n},
$$

where the common transfer integral value defines the system energy scale (i.e., $t \equiv 1$ );

(ii) the sequence of bonds along the chain $\left\{t_{n, n+1}\right\}$ (Figures 13(c) and 13(d)); these arrangements are referred to as the induced (Figure 13(c)) and the standard (Figure 13(d)) transfer (or off-diagonal) models, respectively. In both cases all the atomic potentials are assumed to be equal $\left(V_{n}=V, \forall n\right)$ and (9) reduces to

$$
t_{n, n+1} \psi_{n+1}+t_{n, n-1} \psi_{n-1}=E \psi_{n}
$$

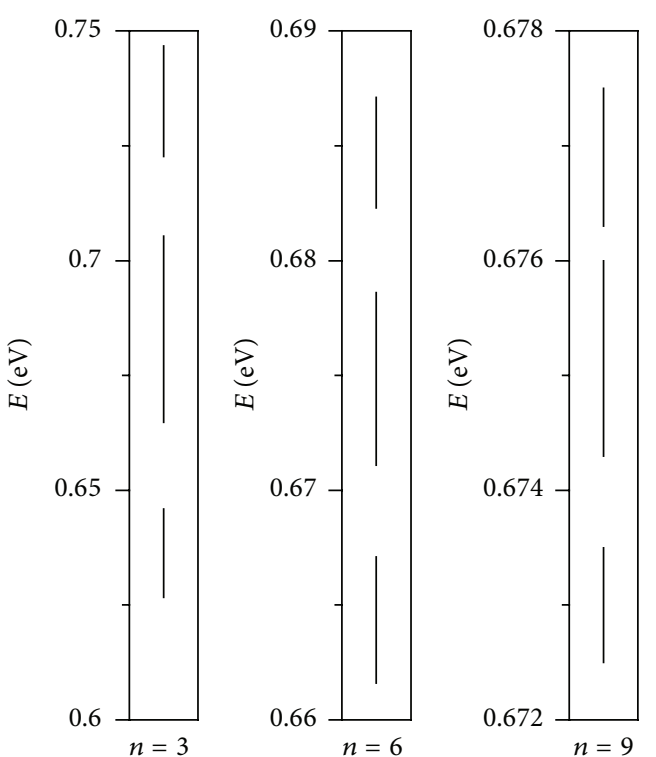

FIGURE 12: Self-similarity in the energy spectrum of a InAs/GaSb Fibonacci superlattice. The left panel shows the whole spectrum for an order $n=3$ superlattice, whereas the central and right panels show a detail of the spectrum for superlattices of orders $n=6$ and $n=9$, respectively (from [47], with permission from IOP Publishing Ltd.).

where, without loss of generality, the origin of energy is set by the atomic potentials (i.e., $V \equiv 0$ );

(iii) both the atoms and bonds sequences; this arrangement corresponds to the general case, and it is generally assumed that the QP sequence of bonds is determined by the QP sequence of atoms, since chemical bonds between different atoms will depend on their chemical nature (Figure 13(a)). As a consequence, the arrangement of transfer integrals $t_{A B}=t_{B A}$ and $t_{A A}$ is synchronized with $\left\{V_{n}\right\}$ in such a way that the $\left\{t_{n, n+1}\right\}$ sequence is still aperiodic but it does not obey the same QP sequence that determines the $\left\{V_{n}\right\}$ sequence (cf. Figures 13(c) and 13(d)).

Due to mathematical simplicity reasons, most early works in the period 1985-1990 focused on the two particular versions of the Schrödinger equation given by the on-site and the standard transfer models. In Table 3 we list the parameters generally used in the study of different binary QP models.

Making use of the trivial relation $\psi_{n}=\psi_{n}$, (9) can be cast in the convenient matrix form:

$$
\left(\begin{array}{c}
\psi_{n+1} \\
\psi_{n}
\end{array}\right)=\left(\begin{array}{cc}
\frac{E-V_{n}}{t_{n, n+1}} & -\frac{t_{n, n-1}}{t_{n, n+1}} \\
1 & 0
\end{array}\right)\left(\begin{array}{c}
\psi_{n} \\
\psi_{n-1}
\end{array}\right) \equiv \mathbf{M}_{n}\left(\begin{array}{c}
\psi_{n} \\
\psi_{n-1}
\end{array}\right),
$$

where $\mathbf{M}_{n}$ is referred to as the local transfer matrix. Thus, for a given system size $N$, the wave function amplitudes can 


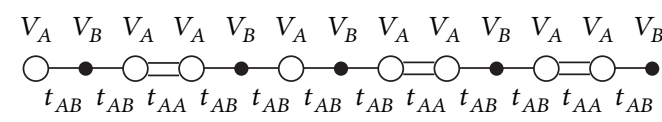

(a)

V

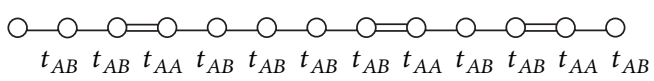

(c)

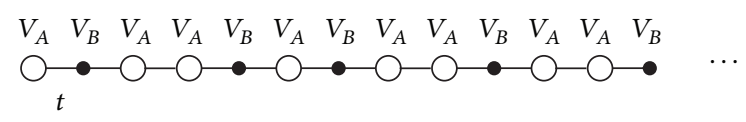

(b)

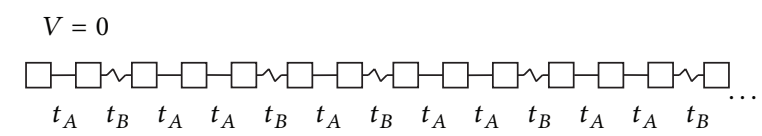

(d)

FIGURE 13: Tight-binding Fibonacci chain models describing the electron dynamics in terms of on-site energies $V_{n}$ and transfer integrals $t_{n, n \pm 1}$ in the (a) general case, (b) on-site (diagonal) model, (c) induced transfer model, and (d) standard transfer (off-diagonal) model.

TABLE 3: Atomic potential and transfer integral parameter values adopted in different models.

\begin{tabular}{lcc}
\hline Model & Potential & Transfer integral \\
\hline General & $V_{A} \equiv \epsilon=-V_{B}$ & $t_{A A} \neq t_{A B} \equiv 1$ \\
On site & $V_{A} \equiv \epsilon=-V_{B}$ & $t_{A A}=t_{A B} \equiv 1$ \\
Standard transfer & $V_{A}=V_{B} \equiv 0$ & $t_{A}=1, t_{B} \neq 1$ \\
Induced transfer & $V_{A}=V_{B} \equiv 0$ & $t_{A A} \neq t_{A B} \equiv 1$ \\
\hline
\end{tabular}

be recursively obtained by successively multiplying the initial values $\psi_{1}$ and $\psi_{0}$, according to the expression:

$$
\left(\begin{array}{c}
\psi_{N+1} \\
\psi_{N}
\end{array}\right)=\prod_{n=N}^{1} \mathbf{M}_{n}\left(\begin{array}{c}
\psi_{1} \\
\psi_{0}
\end{array}\right) \equiv \mathscr{M}_{N}(E)\left(\begin{array}{l}
\psi_{1} \\
\psi_{0}
\end{array}\right)
$$

where $\mathscr{M}_{N}(E)$ is the so-called global transfer matrix. Therefore, to solve the Schrödinger equation is completely equivalent to calculating products of transfer matrices, and the QP order of the system is naturally encoded in the particular order of multiplication of these transfer matrices to give $\mathscr{M}_{N}(E)$. In this way, the noncommutative character of the matrix product endows the global transfer matrix with a fundamental role on the description of QP order effects in the transport properties of QPS.

Indeed, within the transfer matrix framework the complexity of a given QPS can be measured by the number of different kinds of local transfer matrices which are necessary to fully describe it as well as by the particular order of appearance of these matrices along the chain. For instance, in the case of the Fibonacci sequence one has two different local transfer matrices in the on-site model (see Figure 13(b))

$$
\mathbf{A} \equiv\left(\begin{array}{cc}
E-\epsilon & -1 \\
1 & 0
\end{array}\right), \quad \mathbf{B} \equiv\left(\begin{array}{cc}
E+\epsilon & -1 \\
1 & 0
\end{array}\right)
$$

where, without loss of generality, the origin of energies is defined in such a way that $V_{A}=\epsilon=-V_{B}$ (Table 3). The number of required local transfer matrices increases to three in the transfer models. Thus, for the standard model we have $\left(t_{A} \equiv 1\right)$

$$
\begin{gathered}
\mathbf{C} \equiv\left(\begin{array}{cc}
E & -1 \\
1 & 0
\end{array}\right), \quad \mathbf{D} \equiv\left(\begin{array}{cc}
E & -t_{B} \\
1 & 0
\end{array}\right), \\
\mathbf{E} \equiv\left(\begin{array}{cc}
E t_{B}^{-1} & -t_{B}^{-1} \\
1 & 0
\end{array}\right),
\end{gathered}
$$

whereas for the induced transfer model one gets

$$
\mathbf{C}, \mathbf{K} \equiv\left(\begin{array}{cc}
E & -\gamma \\
1 & 0
\end{array}\right), \quad \mathbf{L} \equiv\left(\begin{array}{cc}
E \gamma^{-1} & -\gamma^{-1} \\
1 & 0
\end{array}\right)
$$

where $\gamma \equiv t_{A A} / t_{A B}$, and the energy scale is given by $t_{A B} \equiv 1$. Finally, the number of local transfer matrices rises to four in the general case; namely,

$$
\mathbf{A}, \mathbf{B}, \mathbf{Z} \equiv\left(\begin{array}{cc}
E-\epsilon & -\gamma \\
1 & 0
\end{array}\right), \quad \mathbf{Y} \equiv\left(\begin{array}{cc}
\gamma^{-1}(E-\epsilon) & -\gamma^{-1} \\
1 & 0
\end{array}\right)
$$

The presence of the matrices $\mathbf{A}$ and $\mathbf{B}$ in the general model indicates that the on-site model can be naturally obtained as a particular case corresponding to the condition $\gamma=1$, which reduces $\mathbf{Z}=\mathbf{Y}=\mathbf{A}$. In a similar way, the induced transfer model can be obtained from the general one by imposing the condition $\epsilon=0$, which reduces $\mathbf{Z}=\mathbf{K}$, $\mathbf{Y}=\mathbf{L}$, and $\mathbf{A}=\mathbf{B}=\mathbf{C}$. On the contrary, the standard transfer model cannot be straightforwardly obtained from the general one, because the transfer integrals sequence $\left\{t_{n, n+1}\right\}$ corresponding to the general case does not coincide with the atomic potential sequence $\left\{V_{n}\right\}$ (Figure 13(c)). Accordingly, the general, on-site, and induced transfer models can be regarded as sharing the same lattice topology, whereas the standard transfer model does not. We also note that the matrices A, B, and $\mathbf{C}$ are unimodular (i.e., their determinants equal unity), while the remaining local transfer matrices are not.

In the case of the on-site model the transfer matrix formalism allows one to establish a one-to-one correspondence between the atomic potentials sequence and the local 
TABLE 4: The first Fibonacci numbers from $j=1$ to $j=24$.

\begin{tabular}{llll}
\hline$F_{1}=1$ & $F_{7}=21$ & $F_{13}=377$ & $F_{19}=6765$ \\
$F_{2}=2$ & $F_{8}=34$ & $F_{14}=610$ & $F_{20}=10946$ \\
$F_{3}=3$ & $F_{9}=55$ & $F_{15}=987$ & $F_{21}=17711$ \\
$F_{4}=5$ & $F_{10}=89$ & $F_{16}=1597$ & $F_{22}=28657$ \\
$F_{5}=8$ & $F_{11}=144$ & $F_{17}=2584$ & $F_{23}=46368$ \\
$F_{6}=13$ & $F_{12}=233$ & $F_{18}=4181$ & $F_{24}=75025$ \\
\hline
\end{tabular}

transfer matrices sequence (see Figure 13(b)) so that the global transfer matrix reads

$$
\mathscr{M}_{N}(E)=\prod_{n=N}^{1} \mathbf{M}_{n}=\cdots \text { BААВААВАВААВА. }
$$

Since $\mathbf{A}$ and $\mathbf{B}$ are both unimodular it can be stated that the global transfer matrix for the on-site model belongs to the $S l(2, \mathbb{R})$ group. It is ready to check that the matrices string in (29) can be recursively obtained by concatenation according to the expression $\mathbf{M}_{n+1}=\mathbf{M}_{n-1} \mathbf{M}_{n}$, starting with $\mathbf{M}_{0}=\mathbf{B}$ and $\mathbf{M}_{1}=\mathbf{A}$, so that, if $N=F_{j}$, where $F_{j}$ is a Fibonacci number obtained from the recursive law $F_{j}=F_{j-1}+F_{j-2}$, with $F_{1}=1$ and $F_{0}=1$, the number of $\mathbf{A}$ matrices is $n_{A}=F_{j-1}$ and the number of $\mathbf{B}$ matrices is $n_{B}=F_{j-2}$. For the sake of information in Table 4 we list the first Fibonacci numbers.

Making use of (26), the global transfer matrix for the standard transfer model reads (see Figure 13(d))

$$
\mathscr{M}_{N}(E)=\prod_{n=N}^{1} \mathbf{M}_{n}=\cdots \operatorname{CDEDECDE} \equiv \cdots \widetilde{\mathbf{B}} \widetilde{\mathbf{A}} \widetilde{\mathbf{A}} \widetilde{\mathbf{A}}
$$

where we have defined $\widetilde{\mathbf{B}} \equiv \mathbf{C}$ and introduced the new matrix [48]:

$$
\widetilde{\mathbf{A}} \equiv \mathbf{D E}=t_{B}^{-1}\left(\begin{array}{cc}
E^{2}-t_{B}^{2} & -E \\
E & -1
\end{array}\right)
$$

which is unimodular. For $N=F_{j}$ there are $n_{A}=F_{j-2}$ matrices of type $\widetilde{\mathbf{A}}$ and $n_{B}=F_{j-3}$ matrices of type $\widetilde{\mathbf{B}}$. Therefore, by introducing the matrices $\widetilde{\mathbf{A}}$ and $\widetilde{\mathbf{B}}$ we are able to express the global transfer matrix corresponding to the standard transfer model in terms of just two different unimodular matrices arranged according to the Fibonacci sequence, as we did for the on-site model. Therefore, $\mathscr{M}_{N}(E)$ also belongs to the $S l(2, \mathbb{R})$ group in this case.

In a similar way, making use of (27), we can express the global transfer matrix corresponding to the induced transfer model as

$$
\begin{aligned}
\mathscr{M}_{N}(E) & =\prod_{n=N}^{1} \mathbf{M}_{n}=\cdots \mathbf{C C K L C K L C C C K L C} \\
& \equiv \cdots \mathbf{S}_{B} \mathbf{S}_{A} \mathbf{S}_{A} \mathbf{S}_{B} \mathbf{S}_{A},
\end{aligned}
$$

where we have introduced the auxiliary unimodular matrices:

$$
\begin{gathered}
\mathbf{S}_{B} \equiv \mathbf{C}^{2}=\left(\begin{array}{cc}
E^{2}-1 & -E \\
E & -1
\end{array}\right), \\
\mathbf{S}_{A} \equiv \mathbf{K L C}=\gamma^{-1}\left(\begin{array}{cc}
E\left(E^{2}-\gamma^{2}-1\right) & \gamma^{2}-E^{2} \\
E^{2}-1 & -E
\end{array}\right),
\end{gathered}
$$

so that $\mathscr{M}_{N}(E)$ belongs to the $S l(2, \mathbb{R})$ group for the induced transfer model as well. In this case, for $N=F_{j}$ we have $n_{A}=$ $F_{j-3}$ matrices $\mathbf{S}_{A}$ and $n_{B}=F_{j-4}$ matrices $\mathbf{S}_{B}$.

Finally, making use of (28) we can translate the potentials sequence $\left\{V_{A}, V_{B}, V_{A}, V_{A}, V_{B}, \ldots\right\}$ describing the atomic order of the general Fibonacci lattice to the local transfer matrices product $\cdots$ ABZYBZYBABZYB describing the behavior of electrons moving through it (see Figure 13(a)). In spite of its greater apparent complexity, we realize that by renormalizing this transfer matrix sequence according to the blocking scheme $\mathbf{R}_{A} \equiv \mathbf{Z Y B}$ and $\mathbf{R}_{B} \equiv \mathbf{A B}$, we get the considerably simplified global transfer matrix:

$$
\mathscr{M}_{N}(E)=\prod_{n=N}^{1} \mathbf{M}_{n}=\cdots \mathbf{R}_{B} \mathbf{R}_{A} \mathbf{R}_{A} \mathbf{R}_{B} \mathbf{R}_{A} .
$$

Thus, we can express the $\mathscr{M}_{N}(E)$ matrix of the Fibonacci general model in terms of just two matrices instead of the original four [49]. The subscripts in the $\mathbf{R}_{v}$ matrices are introduced to emphasize the fact that the renormalized transfer matrix sequence is also arranged according to the Fibonacci sequence and, consequently, the topological order present in the original lattice is preserved by the renormalization process. In fact, if $N=F_{j}$ is the number of original lattice sites, it can then be shown by induction that the renormalized matrix sequence contains $n_{A}=F_{j-3}$ matrices $\mathbf{R}_{A}$ and $n_{B}=F_{j-4}$ matrices $\mathbf{R}_{B}$. Quite remarkably, the renormalized matrices

$$
\begin{gathered}
\mathbf{R}_{B}=\left(\begin{array}{cc}
E^{2}-\epsilon^{2}-1 & \epsilon-E \\
\epsilon+E & -1
\end{array}\right), \\
\mathbf{R}_{A}=\gamma^{-1}\left(\begin{array}{cc}
R_{11}(E) & \gamma^{2}-(E-\epsilon)^{2} \\
E^{2}-\epsilon^{2}-1 & \epsilon-E
\end{array}\right),
\end{gathered}
$$

with $R_{11}(E)=(E+\epsilon)\left[(E-\epsilon)^{2}-\gamma^{2}\right]+\epsilon-E$, are both unimodular so that $\mathscr{M}_{N}(E)$ belongs to the $S l(2, \mathbb{R})$ group in the general Fibonacci model as well.

As we see, the matrix elements of the different renormalized matrices are polynomials of the electron energy so that one may expect that these matrices could adopt particularly simple forms for certain energy values. To explore such a possibility in a systematic way it is convenient to explicitly 
evaluate the commutators corresponding to the different Fibonacci lattices shown in Figure 13 [49, 50]:

$$
\begin{gathered}
{\left[\mathbf{R}_{A}, \mathbf{R}_{B}\right]=\frac{\epsilon\left(1+\gamma^{2}\right)-E\left(1-\gamma^{2}\right)}{\gamma}\left(\begin{array}{cc}
1 & 0 \\
E+\epsilon & -1
\end{array}\right),} \\
{\left[\mathbf{S}_{A}, \mathbf{S}_{B}\right]=\frac{E\left(\gamma^{2}-1\right)}{\gamma}\left(\begin{array}{cc}
1 & 0 \\
E & -1
\end{array}\right),} \\
{[\widetilde{\mathbf{A}}, \widetilde{\mathbf{B}}]=\frac{t_{B}^{2}-1}{t_{B}}\left(\begin{array}{ll}
0 & 1 \\
1 & 0
\end{array}\right), \quad[\mathbf{A}, \mathbf{B}]=2 \epsilon\left(\begin{array}{ll}
0 & 1 \\
1 & 0
\end{array}\right) .}
\end{gathered}
$$

Thus, we see that both the on-site and the standard transfer models are intrinsically noncommutative, for their commutators only vanish in the trivial $V_{A}=V_{B} \equiv 0$ or $t_{A A}=t_{A B} \equiv 1$ cases, respectively. On the contrary, in the induced transfer model the $\mathbf{S}_{v}$ matrices commute for the energy value $E=0 .{ }^{16}$ In that case,

$$
\mathbf{S}_{B}(0)=\left(\begin{array}{cc}
-1 & 0 \\
0 & -1
\end{array}\right)=-\mathbf{I}, \quad \mathbf{S}_{A}(0)=\left(\begin{array}{cc}
0 & \gamma \\
-\gamma^{-1} & 0
\end{array}\right)
$$

and (34) can be expressed as

$$
\begin{aligned}
\mathscr{M}_{N}(0) & =\mathbf{S}_{B}^{n_{B}}(0) \mathbf{S}_{A}^{n_{A}}(0) \\
& =(-1)^{n_{B}}\left(\begin{array}{cc}
-U_{n_{A}-2}(0) & \gamma U_{n_{A}-1}(0) \\
\gamma^{-1} U_{n_{A}-1}(0) & -U_{n_{A}-2}(0)
\end{array}\right),
\end{aligned}
$$

where we have made use of the Cayley-Hamilton theorem. ${ }^{17}$ Equation (38) guarantees that the $E=0$ state belongs to the energy spectrum, since $\left|\operatorname{tr} \mathscr{M}_{N}(0)\right|=\left|2 U_{n_{A}-2}(0)\right|=$ $\left|2 \sin \left(\left(n_{A}-1\right) \pi / 2\right)\right| \leq 2, \forall n_{A}$. On the other hand, making use of the relationships $U_{2 n}(0)=(-1)^{n}$ and $U_{2 n+1}(0)=0$, we realize that (38) can take on two different forms, depending on the parity of the $n_{A}=F_{j-3}$ integer; namely,

$$
\mathscr{M}_{N}(0)= \begin{cases}(-1)^{n_{B}} \mathbf{I}, & n_{A} \text { even } \\ (-1)^{n_{B}} \mathbf{S}_{A}(0), & n_{A} \text { odd } .\end{cases}
$$

Thus, the wave function has a simple form and takes on values \pm 1 or $\pm \gamma^{ \pm 1}$ (assuming $\psi_{0}=\psi_{1}=1$ ). The $A$ sites have $\left|\psi_{n}\right|^{2}=1$ or $\gamma^{-2}$, whereas the $B$ sites have $\left|\psi_{n}\right|^{2}=1$ or $\gamma^{2}$ [50]. Accordingly, this is an extended state, which propagates through the Fibonacci induced transfer model.

Finally, according to expression (36), there exists always one energy satisfying the relation:

$$
E_{*}=\epsilon \frac{1+\gamma^{2}}{1-\gamma^{2}},
$$

for any realization of the general model (i.e., for any combination of $\epsilon \neq 0$ and $\gamma \neq 1$ values). For these energies the condition $\left[\mathbf{R}_{A}, \mathbf{R}_{B}\right]=\mathbf{0}$ is fulfilled and, making use of the Cayley-Hamilton theorem, the global transfer matrix of the system, $\mathscr{M}_{N}\left(E_{*}\right) \equiv \mathbf{R}_{A}^{n_{A}} \mathbf{R}_{B}^{n_{B}}$, can be explicitly evaluated. From the knowledge of $M_{N}\left(E_{*}\right)$ the condition for the considered energy value to be in the spectrum, $\left|\operatorname{tr}\left[\mathscr{M}_{N}\left(E_{*}\right)\right]\right| \leq 2$, can be readily checked. We will discuss in detail the properties of these states in Sections 3.3 and 4.2.
2.2. Fractal Lattices. Prior to the discovery of QCs it was suggested by some authors that fractal structures, which instead of the standard translation symmetry exhibit scale invariance, may be suitable candidates to bridge the gap between crystalline and disordered materials [51]. Such a possibility was further elaborated in subsequent works on inhomogeneous fractal glasses [52, 53], a class of structures which are characterized by a scaling distribution of pore sizes and a great variety in the site environments. From this perspective it is interesting to compare the physical properties related to these two novel representatives of the orderings of matter, namely, QCs and fractals.

Thus, both numerical and analytical evidences of localized, critical, and extended wave functions alternating in a complicated way have been reported for several fractal models [54-62]. In addition, it was reported that the interplay between the local symmetry and the self-similar nature of a fractal gives rise to the existence of persistent superlocalized modes in the frequency spectrum [63]. This class of states arise as a consequence of the fact that the minimum path between two points on a fractal does not always follow a straight line [64]. Consequently, the general question regarding whether the nature of the states might be controlled by the fractality of the substrate is an interesting open question, well deserving further scrutiny.

Let us start by considering the celebrated triadic Cantor model, which can be obtained from the substitution rule $A \rightarrow A B A$ and $B \rightarrow B B B$, leading to the sequences

$$
\begin{aligned}
A B A & \longrightarrow A B A B B B A B A \\
& \longrightarrow A B A B B B A B A B B B B B B B B B A B A B B B A B A
\end{aligned}
$$

The number of letters contained in the $k$ order fractal generation is $N=3^{k}$. By inspecting these strings we realize that these sequences differ from the binary QP sequences considered in Section 2.2 in the sense that, as the system grows on, the subclusters of $B$ s grow in size to span the entire 1D space. Therefore, in the thermodynamic limit the lattice may be looked upon as an infinite string of $B$ atoms, punctuated by $A$ atoms which play the role of impurities located at specific sites determined by the Cantor sequence $[60,65]$.

The Schrödinger equation corresponding to the on-site version of the triadic Cantor lattice can be expressed in terms of a global transfer matrix composed of the two local transfer matrices $\mathbf{A}$ and $\mathbf{B}$ given by (25), as follows:

$$
\begin{gathered}
\mathscr{M}_{3}(E)=\mathbf{A B A} \\
\mathscr{M}_{9}(E)=\mathbf{A B A B}^{3} \mathbf{A B A} \\
\mathscr{M}_{27}(E)=\mathbf{A B A B}^{3} \mathbf{A B A B}^{9} \mathbf{A B A B}^{3} \mathbf{A B A},
\end{gathered}
$$

One then sees that, for those energy values satisfying the condition $\mathbf{B}^{3}= \pm \mathbf{I}$, these global transfer matrices reduce to 


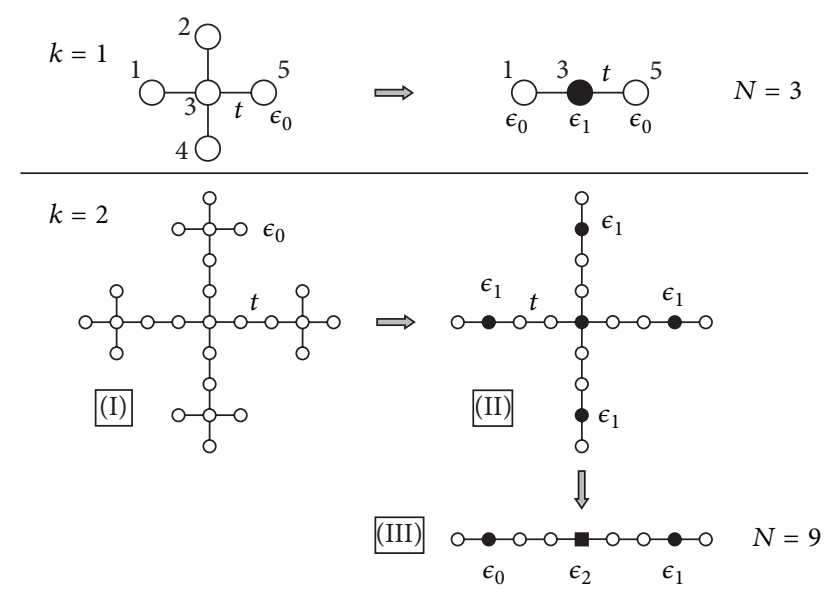

FIGURE 14: Tight-binding homogeneous Vicsek fractal model describing the electron dynamics in terms of on-site energies $\epsilon_{0}$ and transfer integrals $t$. The fractal generation order is given by the integer $k$, while $N$ stands for the number of atoms in the decimated chains.

that corresponding to a periodic structure with a unit cell $A B A{ }^{18}$ Accordingly, if the energies satisfying the condition $\mathbf{B}^{3}= \pm \mathbf{I}$ also belong to the spectrum of the $A B A$ periodic lattice (i.e., they satisfy the property $|\operatorname{tr}(\mathbf{A B A})| \leq 2$ ), then these energies will correspond to extended states. Making use of the Cayley-Hamilton theorem the above resonance condition can be expressed in terms of the matrix equation:

$$
\mathbf{B}^{3}=\left(\begin{array}{ll}
U_{3}(x) & -U_{2}(x) \\
U_{2}(x) & -U_{1}(x)
\end{array}\right) \equiv\left(\begin{array}{cc} 
\pm 1 & 0 \\
0 & \pm 1
\end{array}\right)
$$

where $U_{3}(x)=8 x^{3}-4 x, U_{2}(x)=4 x^{2}-1$, and $x=$ $(E+\epsilon) t^{-1} / 2$. By solving (43) one obtains $x=-1 / 2$, when $\mathbf{B}^{3}=\mathbf{I}$, and $x=1 / 2$ when $\mathbf{B}^{3}=-\mathbf{I}$. In a similar way, one can get other extended states by setting $\mathbf{B}^{3^{k}}= \pm \mathbf{I}$, with $k>1$, and solving the resulting polynomial equation. Thus, the on-site triadic Cantor set admits a countable set of extended states, whose number progressively increases as the system size increases. This result properly illustrates that the scale invariance symmetry related to self-similar topology characteristic of a Cantor lattice favours the presence of extended states propagating through the structure.

An illustrative fractal lattice extending in more than one dimension is provided by the so-called Vicsek lattice. The pattern corresponding to its two first generations is shown in Figure 14, where we see that its basic building block is formed by a cross composed of five identical atoms (on site energy $\epsilon_{0}$ ) coupled to the central one with identical transfer integrals $t$. Quite remarkably, this fractal lattice can be exactly mapped into a 1D chain of atoms by systematically decimating the upper and lower branches around the central site at any fractal generation, as it is illustrated on the right hand of Figure 14 for first- and second-generation lattices. The resulting effective $1 \mathrm{D}$ decimated chain is composed of a set of $N=3^{k}$ atoms with different on-site energy values, which are coupled to each other by identical transfer integrals $t$.
The resulting decimated lattice exhibits a hierarchical structure so that the successive values of the on-site energy series $\epsilon_{0}, \epsilon_{1}, \epsilon_{2}, \ldots$ arrange themselves in a self-similar pattern, where $\varepsilon_{k}$ arises out of the decimation of bigger and bigger clusters of atoms around the central sites at each $k$ generation order of the fractal, and are given by the fraction series:

$$
\begin{gathered}
\epsilon_{1}=\epsilon_{0}+\frac{2 t^{2}}{E-\epsilon_{0}}, \\
\epsilon_{2}=\epsilon_{1}+\frac{2 t^{2}}{E-\epsilon_{1}-t^{2} /\left(E-\epsilon_{1}-t^{2} /\left(E-\epsilon_{0}\right)\right)},
\end{gathered}
$$

By inspecting Figure 14 we realize that by imposing the condition $\epsilon_{1}=\epsilon_{0}$ the decimated lattice corresponding to the first generation Vicsek fractal reduces to a monatomic periodic chain, whereas by imposing the condition $\epsilon_{2}=\epsilon_{1}$ the decimated lattice corresponding to the second generation Vicsek fractal reduces to a binary periodic chain, the unit cell of which is precisely given by the $k=1$ decimated chain. Accordingly, the eigenstates corresponding to these resonance energies will be extended states in their corresponding effective periodic chains. Obviously, the same procedure can be extended to higher order generations to reduce the original hierarchical lattice to a periodic lattice containing as many different types of atoms as the generation order. In this regard, one may consider that the topological complexity of the original Vicsek fractal is properly translated to a higher chemical diversity as a consequence of the renormalization processes. Therefore, as the Vicsek fractal grows larger we can obtain a numerable infinite set of extended states in the thermodynamic limit. The energies corresponding to these states can be obtained from (44) by imposing the condition $\epsilon_{k+1}=\epsilon_{k}, k \geq 1$, iteratively. The same condition can be arrived at by considering the local transfer matrices corresponding to the on-site hierarchical lattice model, which have the form:

$$
\mathbf{V}_{k}=\left(\begin{array}{cc}
\frac{E-\epsilon_{k}}{t} & -1 \\
1 & 0
\end{array}\right)
$$

and calculating the commutator:

$$
\left[\mathbf{V}_{k+1}, \mathbf{V}_{k}\right]=\frac{\epsilon_{k+1}-\epsilon_{k}}{t}\left(\begin{array}{ll}
0 & 1 \\
1 & 0
\end{array}\right)
$$

which only commutes when $\epsilon_{k+1}=\epsilon_{k}$. Since the global transfer matrix of the decimated $1 \mathrm{D}$ chain is given as a product of local transfer matrices of the form:

$$
\begin{gathered}
\mathscr{M}_{3}(E)=\mathbf{V}_{0} \mathbf{V}_{1} \mathbf{V}_{0} \\
\mathscr{M}_{9}(E)=\mathbf{V}_{0} \mathbf{V}_{1} \mathbf{V}_{0} \mathbf{V}_{0} \mathbf{V}_{2} \mathbf{V}_{0} \mathbf{V}_{0} \mathbf{V}_{1} \mathbf{V}_{0}
\end{gathered}
$$




$$
\begin{gathered}
\mathscr{M}_{27}(E)=\mathbf{V}_{0} \mathbf{V}_{1} \mathbf{V}_{0} \mathbf{V}_{0} \mathbf{V}_{2} \mathbf{V}_{0} \mathbf{V}_{0} \mathbf{V}_{1} \mathbf{V}_{0} \mathbf{V}_{0} \mathbf{V}_{1} \mathbf{V}_{0} \mathbf{V}_{0} \mathbf{V}_{3} \\
\times \mathbf{V}_{0} \mathbf{V}_{0} \mathbf{V}_{1} \mathbf{V}_{0} \mathbf{V}_{0} \mathbf{V}_{1} \mathbf{V}_{0} \mathbf{V}_{0} \mathbf{V}_{2} \mathbf{V}_{0} \mathbf{V}_{0} \mathbf{V}_{1} \mathbf{V}_{0} \\
\vdots \\
\mathscr{M}_{3^{k+1}}(E)=\mathscr{M}_{3^{k-1}}(E) \mathscr{M}_{3^{k+1}}(E) \mathscr{M}_{3^{k-1}}(E)
\end{gathered}
$$

the hierarchical distribution of the renormalized on-site energies in the decimated lattice guarantees that this hierarchical structure is inherited by the corresponding extended states satisfying the commutation condition stated above.

As a final model example we will consider the tightbinding model on the Koch lattice introduced by Andrade and Schellnhuber [66]. The motivation for this choice stems from the fact that this model Hamiltonian can also be exactly mapped onto a linear chain and the corresponding electron dynamics expressed in terms of just two kinds of renormalized transfer matrices. In this way we can use the same algebraic approach discussed in the study of electron dynamics in general Fibonacci systems in the previous section. The model is sketched in Figure 15, and its tightbinding Hamiltonian is given by [66]

$$
\begin{aligned}
H=\sum_{n}\{|n\rangle\langle n+1|+| n\rangle\langle n-1|+\lambda f(n) \\
\times[|n-1\rangle\langle n+1|+| n+1\rangle\langle n-1|]\},
\end{aligned}
$$

where $\lambda$ is the cross-transfer integral introduced by Gefen et al. [67] (indicated by dashed lines in Figure 15(a)) and

$$
f(n)=\delta(0, n)+\sum_{s}^{k-1} \delta\left(\frac{4^{s}}{2}, n\left(\bmod 4^{s}\right)\right),
$$

with $k \geq 2$ and $-4^{k} / 2 \leq n \leq 4^{k} / 2$, describing the effective next-nearest-neighbor interaction in the $k$ th stage of the fractal growth process. The main effect of allowing electron hopping across the folded lattice is the existence of sites with different coordination numbers along the lattice, a characteristic feature of fractals which is not shared by QP lattices. Depending on the value of their respective coordination numbers we can distinguish twofold (circles), threefold (full triangles) and fourfold (squares) sites. We then notice that even sites are always twofold, a fact which allows us to renormalize the original lattice mapping it into the linear form sketched in Figure 15(b) [66]. The transfer integrals represented by single bonds appear always isolated from one another. The transfer integrals represented by double bonds can appear either isolated or forming trimers. Consequently, there are three possible site environments in the renormalized Koch lattice which, in turn, define three possible types of local transfer matrices, labelled F, G, and $\mathbf{H}$ in Figure 15(b). Now, by introducing the matrices $\mathbf{P} \equiv \mathbf{H G}$ and $\mathbf{P Q} \equiv \mathbf{F F}$, it can be shown by induction that the global transfer matrix at any given arbitrary stage $k$ of the fractal growth process, $\mathscr{M}_{k}$, can be iteratively related to that corresponding to the previous stage, $\mathscr{M}_{k-1}$, by the expression [66]:

$$
\mathbf{P}^{-1} \mathscr{M}_{k}=\mathscr{M}_{k-1}^{2} \mathbf{Q} \mathscr{M}_{k-1}^{2} \text {, }
$$

with $k \geq 2$ and $\mathscr{M}_{1} \equiv \mathbf{P}$. In this way, the topological order of the lattice is translated to the transfer matrices sequence describing the electron dynamics in a natural way. The matrices $\mathbf{P}$ and $\mathbf{Q}$ are unimodular for any choice of $\lambda$ and for any value of the electron energy, $E$, and their commutator reads [55]

$$
\begin{aligned}
{[\mathbf{P}, \mathbf{Q}]=} & \frac{\lambda E\left(E^{2}-2\right)(2+\lambda E)}{r^{3}} \\
& \times\left(\begin{array}{c}
\left(2-E^{2}\right) r \\
\left(1-E^{2}\right)\left(E^{2}-3\right)
\end{array}\left(\begin{array}{c}
\left.E^{2}-2\right) r
\end{array}\right),\right.
\end{aligned}
$$

where $r \equiv 1+\lambda E$, and we have defined the origin of energies in such a way that the transfer integrals along the original chain equal unity. By comparing (51) with the commutator $\left[\mathbf{R}_{A}, \mathbf{R}_{B}\right]$ for the general Fibonacci lattice given by (36) we see that the Koch fractal lattice has a larger number of resonance energies, namely, $E=0, E= \pm \sqrt{2}$, and $E=-2 / \lambda$, as compared to the single $E_{*}$ value obtained for the Fibonacci one.

2.3. Quasiperiodic Optical Devices. In order to fully appreciate the fingerprints of long-range aperiodic order, the study of classical waves propagating through an aperiodic substrate offers a number of advantages over the study of quantum elementary excitations.

Consequently, light transmission through aperiodic media has deserved an ever-increasing attention in order to understand the interplay between optical properties and the underlying aperiodic order of the substrate $[18,68-71]$. To this end, the mathematical analogy between Schrödinger equation (5) and Helmholtz equation describing a monochromatic electromagnetic wave of frequency $\omega$ propagating in a lossless, dispersionless medium with a variable refractive index profile $n(x)$

$$
\frac{d^{2} E}{d x^{2}}+\left[\frac{\omega^{2}}{c^{2}} n^{2}(x)-k_{\|}^{2}\right] \mathscr{E}=0,
$$

where $\mathscr{E}$ is the transversal component of the electric field, $k_{\|}$is the wave vector in the XY plane (perpendicular to the propagation direction $z$ ), and $c$ is the vacuum speed of light, provides a powerful tool to relate previous knowledge about electron motion in superlattices to electromagnetic waves propagating in multilayers (Figure 16). We note that the refractive index of the different layers is the physical magnitude relating the aperiodic sequence describing the stacking order along the multilayer (Figure 16(a)) and the resulting aperiodic function describing the multilayer's optical profile (Figure 16(b)). Thus, the isomorphism of Shrödinger and Helmholtz equations provides a helpful analogy involving basic concepts in modern optoelectronics [72].

\section{Nature of States in Aperiodic Systems}

The notion of critical wave function has evolved continuously since its introduction in the study of QP systems (see Section 1.2), leading to a somewhat confusing situation. For instance, references to self-similar, chaotic, lattice-like, or 


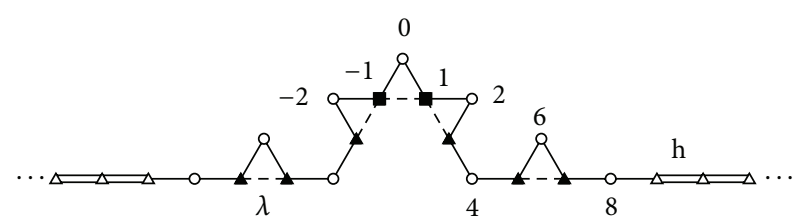

(a)

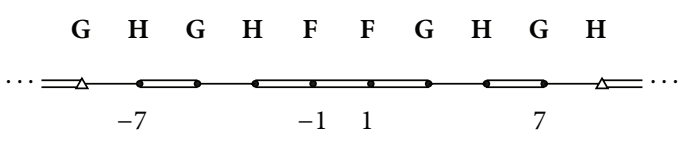

(b)

FIGURE 15: (a) Sketch of the Koch lattice model considered. The different sites are labeled by integers. (b) Sketch of the renormalization scheme mapping the Koch lattice into a linear chain (from [55], with permission from Maciá @ 1998 by the American Physical Society).

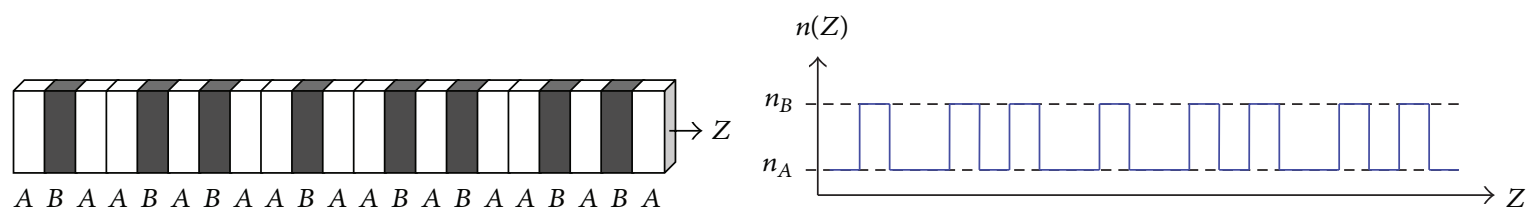

(a)

(b)

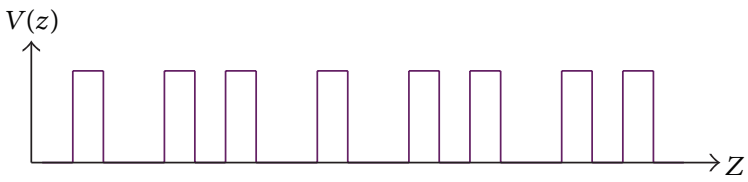

(c)

FIGURE 16: (a) Sketch of a Fibonacci dielectric multilayer grown along the $z$ direction, (b) refractive index profile $n(z)$ for an electromagnetic wave propagating through the structure and (c) electronic potential profile $V(z)$ for an electron propagation along the growth direction (from [68], Maciá, with permission from IOP Publishing Ltd.).

quasilocalized wave functions can be found in the literature depending on the different criteria adopted to characterize them [48, 73-75]. Generally speaking, critical states exhibit a rather involved oscillatory behavior, displaying strong spatial fluctuations which show distinctive self-similar features (Figure 5). Thus, the wave function is peaked on short chain sequences but peaks reappear far away on chain sequences showing the same lattice ordering. As a consequence, the notion of an envelope function, which has been the most fruitful in the study of both extended and localized states, is mathematically ill defined in the case of critical states, and other approaches are required to properly describe them and to understand their structure.

On the other hand, as we have seen in Section 1.4, from a rigorous mathematical point of view, the nature of a state is uniquely determined by the measure of the spectrum to which it belongs. In this way, since it has been proven that a number of QPS have purely singular continuous energy spectra, we must conclude that the associated electronic states cannot be, strictly speaking, extended in Bloch's sense. However, this fact does not necessarily imply that states belonging to singular continuous spectra of different QPS will behave in exactly the same way from a physical viewpoint.

In fact, electronic states can be properly classified according to their related transport properties. Thus, conducting states in crystalline systems are described by periodic Bloch functions, whereas insulating systems exhibit exponentially decaying functions corresponding to localized states. Within this scheme the notion of critical states is somewhat imprecise, because critical states exhibit strong spatial fluctuations at different scales. In this regard, a first step towards a better understanding of critical states was provided by the demonstration that the amplitudes of critical states in onsite Fibonacci lattices do not tend to zero at infinity but are bounded below through the system [28]. This result suggests that the physical behavior of critical states is more similar to that corresponding to extended states than to localized ones. Indeed, the possible existence of extended states in several kinds of aperiodic systems has been discussed in the last years spurring the interest in the precise nature of critical wave functions and their role in the physics of aperiodic systems $[49,50,55,73,76-79]$. As a result, arguments supporting the convenience of widening the very notion of extended state in aperiodic systems to include critical states which are not Bloch functions have been put forward $[49,55]$.

3.1. Eigenstates in On-Site Fractal Lattices. How do the eigenfunctions of self-similar fractal lattices compare with those reported for the Aubry-André model at the critical point? In Figure 17(a) we plot the wave function amplitudes for one of the solutions of (43) for a lattice with $N=3^{5}=243$ lattice sites. The distribution shows an interesting pattern, which mimics the topological atomic arrangement of the lattice itself in the sense that all peaks group close together in clusters containing 1,3 , or 27 peaks, which are also powers of three, like what occurs for the $B$ s strings interspersed in the lattice. For this reason, these types of states are sometimes referred to as lattice-like in the literature. ${ }^{19}$ When comparing 


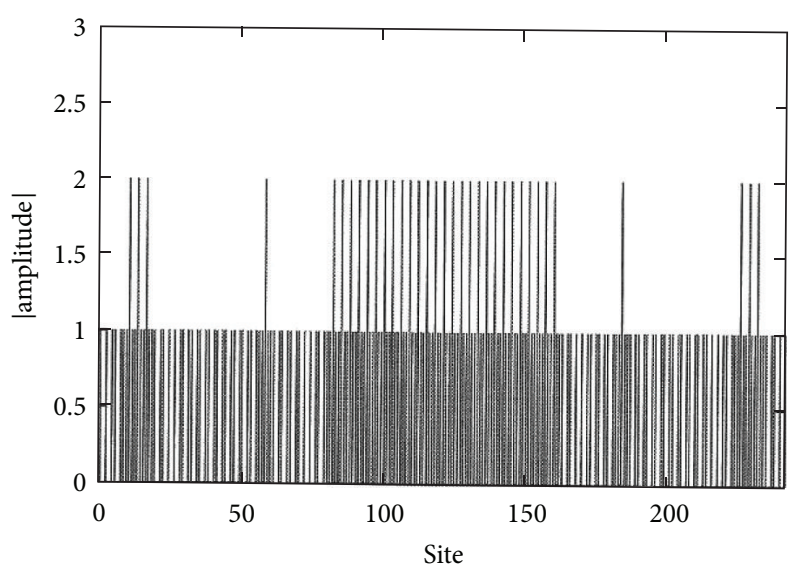

(a)

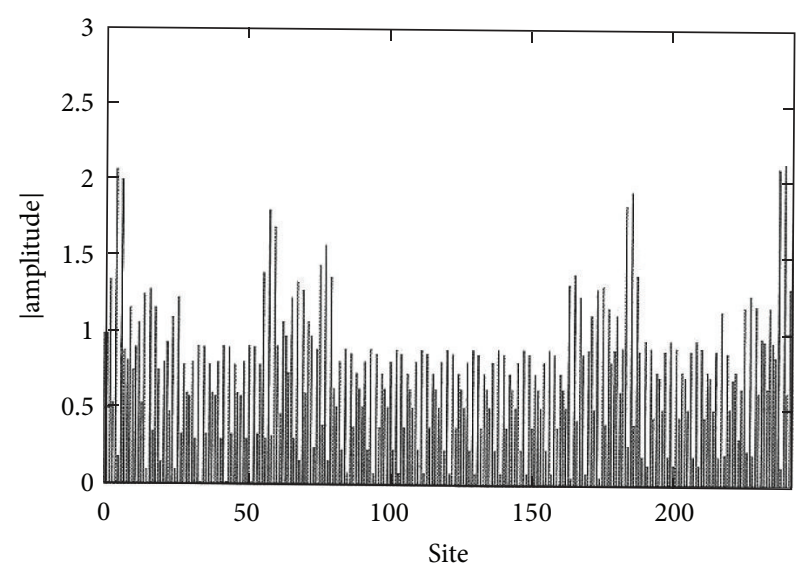

(b)

FIGURE 17: (a) A lattice-like distribution of amplitudes for the extended state $E=0$ in the on-site model of a $N=243$ triadic Cantor lattice. (b) An extended wave function corresponding to the energy $E=-1.3473$ obtained by setting $\mathbf{B}^{9}=-\mathbf{I}$. In both cases $V_{A}=0, V_{B}=-1$ and $t=1$ (adapted from [60], courtesy of Arunava Chakrabarti, with permission from Elsevier).

the wave function distribution amplitudes of this state for lattices of different lengths, it was reported that the latticelike feature recurrently appears and disappears depending on the generation order of the fractal lattice, being present for those lattices whose length is given by the series $N=$ $3^{2 k+1}$, with $k \geq 1$ [60]. For other energy values one obtains extended states which, however, do not generally exhibit the lattice-like property as it is shown in Figure 17(b). Therefore, the lattice-like property is by no means a generic property of extended states in triadic Cantor lattices. In addition, by comparing Figures 5 and 17 we see that the wave functions reported for Cantor lattices lack the characteristic self-similar arrangements of peaks observed in the critical states of the Aubry-André model.

3.2. Self-Similar Wave Functions in Fibonacci Transfer Models. The wave function distribution amplitudes for the state located at the band centre $(E=0)$ in the Fibonacci standard transfer model (see Figure 13(d)) were earlier studied in detail by Kohmoto and coworkers and it is shown in Figure 18. One can readily appreciate a series of main peaks which are sequentially found as $n$ is increased. The peak values are given by powers of the parameter $t_{B} / t_{A}$ measuring the ratio between the long and short bonds (in Figure $18 t_{B}=2$, so that the peaks take on $2^{m}$ values). In order to analyze the spatial distribution of the wave function amplitudes we will split the lattice sites into two complementary sets depending on whether $n$ is (or not) a Fibonacci number. Due to the self-similar structure of Fibonacci lattices the global transfer matrix for lattice sites corresponding to Fibonacci numbers can be iteratively obtained from the relation [80]:

$$
\mathscr{M}_{F_{j+1}}(E)=\mathscr{M}_{F_{j-1}}(E) \mathscr{M}_{F_{j}}(E), \quad j \geq 2 .
$$

On the other hand, making use of the property that any integer can be expressed as a sum of Fibonacci numbers in the form $n=F_{l_{1}}+F_{l_{2}}+\cdots+F_{l_{i}}$, with $l_{1}>l_{2}>\cdots>l_{i} \in \mathbb{N}$, the global transfer matrix for a non-Fibonacci $n$ lattice site is given by

$$
\mathscr{M}_{n}(E)=\mathscr{M}_{F_{l_{i}}}(E) \cdots \mathscr{M}_{F_{l_{2}}}(E) \mathscr{M}_{F_{l_{1}}}(E) .
$$

Let us first consider the lattice sites corresponding to Fibonacci numbers. Since we are considering the $E=0$ state, (31) reads

$$
\begin{gathered}
\mathscr{M}_{1}(0)=\widetilde{\mathbf{B}}(0)=\left(\begin{array}{cc}
0 & -1 \\
1 & 0
\end{array}\right), \\
\mathscr{M}_{2}(0)=\widetilde{\mathbf{A}}(0)=-\left(\begin{array}{cc}
t_{B} & 0 \\
0 & t_{B}^{-1}
\end{array}\right) .
\end{gathered}
$$

Then, making use of (53), one gets

$$
\begin{gathered}
\mathscr{M}_{3}(0)=\mathscr{M}_{1}(0) \mathscr{M}_{2}(0)=\left(\begin{array}{cc}
0 & t_{B}^{-1} \\
-t_{B} & 0
\end{array}\right), \\
\mathscr{M}_{5}(0)=\mathscr{M}_{2}(0) \mathscr{M}_{3}(0)=\left(\begin{array}{cc}
0 & -1 \\
1 & 0
\end{array}\right)=\mathscr{M}_{1}(0), \\
\mathscr{M}_{8}(0)=\mathscr{M}_{3}(0) \mathscr{M}_{5}(0)=\left(\begin{array}{cc}
t_{B}^{-1} & 0 \\
0 & t_{B}
\end{array}\right), \\
\mathscr{M}_{13}(0)=\mathscr{M}_{5}(0) \mathscr{M}_{8}(0)=\left(\begin{array}{cc}
0 & -t_{B} \\
t_{B}^{-1} & 0
\end{array}\right), \\
\mathscr{M}_{21}(0)=\mathscr{M}_{8}(0) \mathscr{M}_{13}(0)=\left(\begin{array}{cc}
0 & -1 \\
1 & 0
\end{array}\right)=\mathscr{M}_{1}(0), \\
\mathscr{M}_{34}(0)=\mathscr{M}_{13}(0) \mathscr{M}_{21}(0)=-\left(\begin{array}{cc}
t_{B} & 0 \\
0 & t_{B}^{-1}
\end{array}\right)=\mathscr{M}_{2}(0) ;
\end{gathered}
$$

accordingly $\mathscr{M}_{55}(0)=\mathscr{M}_{21}(0) \mathscr{M}_{34}(0)=\mathscr{M}_{1}(0) \mathscr{M}_{2}(0)=$ $\mathscr{M}_{3}(0)$, and the entire sequence of global transfer matrices repeats once again. Therefore, we get the six-cycle property $\mathscr{M}_{F_{j+6}}(0)=\mathscr{M}_{F_{j}}(0)$, relating the global transfer matrices of 


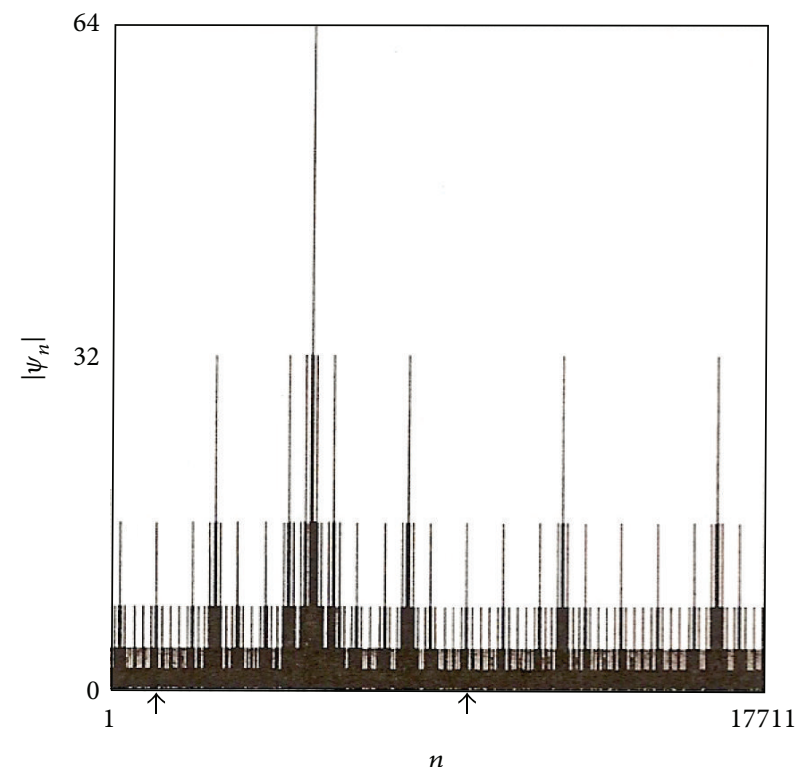

(a)

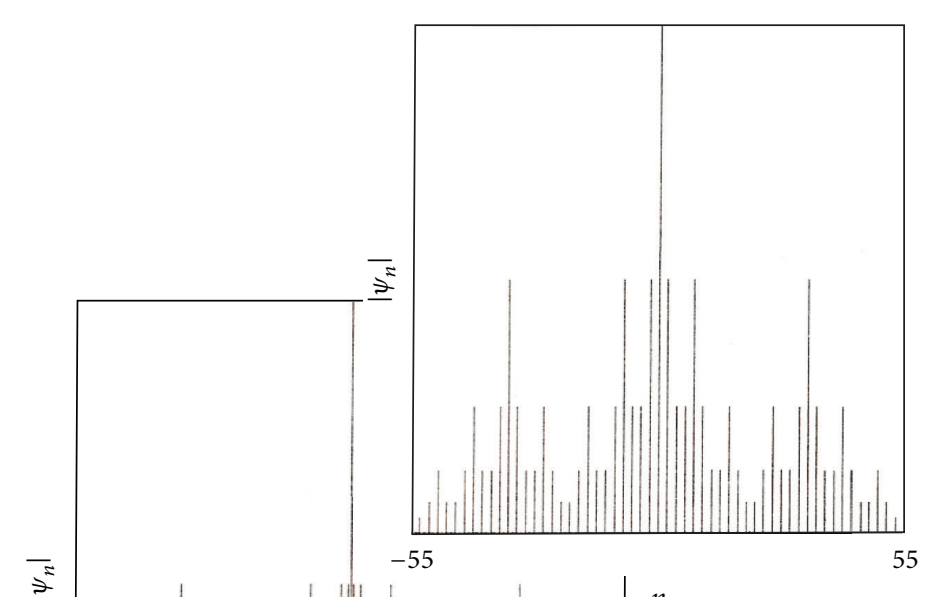

(d)

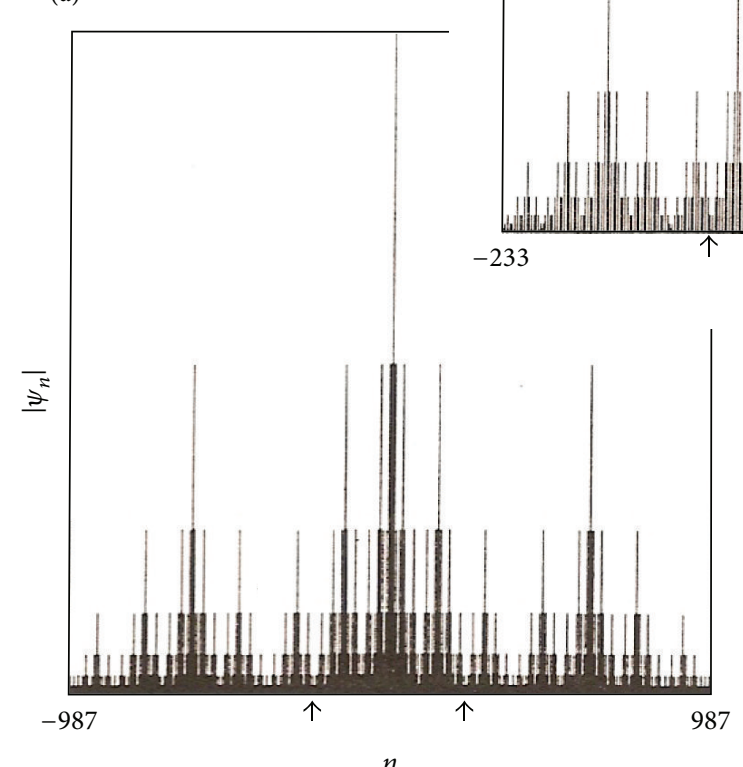

(b)

FIGURE 18: Self-similar features in the wave function located at the center of the spectrum, $E=0$, in the Fibonacci standard transfer model with $t_{B}=2$. In (a) the wave function for a lattice composed of $F_{21}=17711$ sites is plotted. A portion of this wave function, comprised within the sites indicated by the arrows around the highest peak located at $n=5472$, is shown in (b). This amplitude distribution is identically reproduced at progressively smaller scales in (c) and (d) after a proper rescaling (and renumbering of the lattice sites) (from [48], with permission from Kohmoto et al. (c) 1987 by the American Physical Society).

progressively longer sections along the Fibonacci chain. The size $N=55=F_{9}$ thus represents the minimum length necessary for the properties stemming from this matrices repeating pattern to manifest themselves. The structure of (56) matrices also guarantees that the wave function amplitudes can only take on the values \pm 1 or $\pm t_{B}^{ \pm 1}$ times $\psi_{0}$ or $\psi_{1}$ at these lattice sites.

The situation is different for lattice sites which do not correspond to Fibonacci numbers. In fact, there are a series of peaks located at the sites given by the formula
$n_{*}=F_{3}+F_{6}+\cdots+F_{3 p}, p=1,2,3, \ldots$, whose corresponding global transfer matrices (for $p>2$ ) read

$$
\begin{aligned}
\mathscr{M}_{16}(0)=\mathscr{M}_{3}(0) \mathscr{M}_{13}(0)=\left(\begin{array}{cc}
t_{B}^{-2} & 0 \\
0 & t_{B}^{2}
\end{array}\right) \\
\mathscr{M}_{71}(0)=\mathscr{M}_{3}(0) \mathscr{M}_{13}(0) \mathscr{M}_{55}(0)=\mathscr{M}_{16}(0) \mathscr{M}_{3}(0) \\
=\left(\begin{array}{cc}
0 & t_{B}^{-3} \\
-t_{B}^{3} & 0
\end{array}\right)
\end{aligned}
$$




$$
\begin{gathered}
\mathscr{M}_{304}(0)=\mathscr{M}_{3}(0) \mathscr{M}_{13}(0) \mathscr{M}_{55}(0) \mathscr{M}_{233}(0) \\
=\mathscr{M}_{71}(0) \mathscr{M}_{13}(0)=\left(\begin{array}{cc}
t_{B}^{-4} & 0 \\
0 & t_{B}^{4}
\end{array}\right), \\
\mathscr{M}_{1291}(0)=\mathscr{M}_{3}(0) \mathscr{M}_{13}(0) \mathscr{M}_{55}(0) \mathscr{M}_{233}(0) \mathscr{M}_{987}(0) \\
=\mathscr{M}_{304}(0) \mathscr{M}_{3}(0)=\left(\begin{array}{cc}
0 & t_{B}^{-5} \\
-t_{B}^{5} & 0
\end{array}\right), \\
\mathscr{M}_{5472}(0)=\mathscr{M}_{3}(0) \mathscr{M}_{13}(0) \mathscr{M}_{55}(0) \mathscr{M}_{233}(0) \mathscr{M}_{987}(0) \\
\times \mathscr{M}_{4181}(0)=\mathscr{M}_{1291}(0) \mathscr{M}_{13}(0)=\left(\begin{array}{cc}
t_{B}^{-6} & 0 \\
0 & t_{B}^{6}
\end{array}\right),
\end{gathered}
$$

where we have made use of (54) and the six-cycle property $\mathscr{M}_{F_{j+6}}(0)=\mathscr{M}_{F_{j}}(0)$. According to (24) at these lattice sites the wave function amplitudes obey the geometric series $\psi_{n_{*}}=$ $t_{B}^{2 p} \psi_{0}$ and $\psi_{n_{*}}=-t_{B}^{2 p+1} \psi_{1}$. These two series of lattice sites give the fundamental structure of the wave function as they determine the series of progressively higher peaks which are encountered sequentially as $n$ is increased [80]. Indeed, as one considers longer and longer lattices one obtains higher and higher peaks, as it is seen in Figure 18(a). This implies that the wave function is not bounded above in the thermodynamic limit $N \rightarrow \infty$.

A typical self-similar structure, which can be described by means of a proper scale transformation of the wave function amplitudes, can be identified around the highest peaks, as it is illustrated in Figures 18(b)-18(d). ${ }^{20}$ The self-similarity of this wave function can be described precisely as follows: if one assumes $\psi_{0}=\psi_{1}=1$, then the modulus of the wave function is invariant under the scale transformation [80]:

$$
\begin{gathered}
n \longrightarrow n \tau^{-3}, \\
|\psi| \longrightarrow|\psi| t_{B}^{-1} \quad|\psi|>1 \\
|\psi| \longrightarrow|\psi| t_{B}, \quad|\psi|<1,
\end{gathered}
$$

where $\tau=(1+\sqrt{5}) / 2$ is the golden mean. Accordingly, the wave function amplitude obeys a power-law distribution of the form $\psi_{n} \simeq n^{\xi}$, with $\xi=\left|\ln \left(t_{B} / t_{A}\right)\right| / \ln \tau^{3}$ (for $t_{B}=2$ and $t_{A}=1$ one gets $\xi=0.480$ ) [80], which defines a local envelope of the wave function around the sites with high peak values. A similar critical exponent for the envelope of the wave function was obtained by introducing a renormalization group method to study the Fibonacci standard transfer model $[81,82]$. The reason for a power-law behavior is basically that the global transfer matrices of the form

$$
\left(\begin{array}{cc}
t_{B}^{-2 m} & 0 \\
0 & t_{B}^{2 m}
\end{array}\right),
$$

corresponding to lattice sites $n_{*}=\sum F_{3 p}$ containing an even number of Fibonacci numbers in the sum (e.g., $n_{*}=16,304$ and 5472 in (57)), have an eigenvalue which is greater than

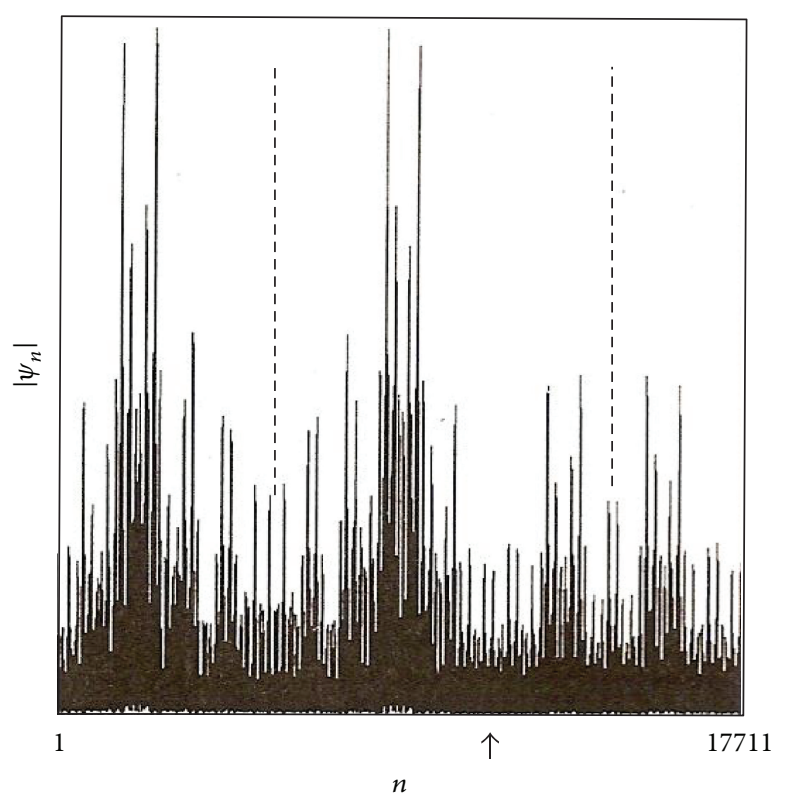

FIGURE 19: Example of a non-self-similar wave function corresponding to $E=0.267958$ in the Fibonacci standard transfer model with $t_{B}=2$ (adapted from [48], with permission from Kohmoto et al. (C) 1987 by the American Physical Society).

unity. $^{21}$ Therefore, although the wave function does not grow and only takes the values $\left\{ \pm 1, \pm\left(t_{B} / t_{A}\right)^{ \pm 1}\right\}$ at Fibonacci sites, it grows as a power-law at non-Fibonacci sites.

Although these exact results have been obtained for the special energy $E=0$, there are many other energies at which the wave functions behave similarly. Those energies are at the centers of the main subbands in the energy spectrum, which progressively appear as the system grows longer. Self-similar wave functions were also reported at the energy spectrum edges, where the global transfer matrices exhibit a two-cycle recurrence [48].

However, as far as 1986, the notion of critical wave function in Fibonacci systems was not yet clearly defined, as it is illustrated by this excerpt by Kohmoto [42]:

There are two types of unusual wave functions: self-similar and chaotic. The self-similarity exists for wave functions corresponding to the center and the edge of each subcluster of the spectrum. The wave function at each center is similar to the exact wave function at $E=0$. In the same way, all the wave functions at the edges are similar to each other. Chaotic states exist almost everywhere in the spectrum. They are the remaining states which are not at the centers or the edges of every subband in the spectrum. These states are not normalizable and do not have apparent scaling properties. The nature of these chaotic states is not well understood yet.

An example of a non-self-similar wave function, characterized by strong spatial fluctuations of the wave function amplitudes, is shown in Figure 19. The overall, long-range 
structure of this wave function can be split into two main regions including $F_{20}=10946$ and $F_{19}=6765$ lattice sites each, which are, respectively, located on the left (right) of the arrow shown in the figure. The wave function amplitudes corresponding to these two regions exhibit distinctive mirror symmetry features with respect to the dashed vertical lines. Although one cannot see any evidence of self-similar patterns in this amplitudes distribution by simple visual inspection, there exist more powerful methods to disclose short-range internal correlations in such a distribution. Two wide procedures used to this end are based on the so-called multifractal and wavelets analysis, respectively (see Section 1.5).

The use of multifractal methods to analyze the electronic states in Fibonacci lattices provided conclusive evidence on the existence of different kinds of critical states, depending on their location in the highly fragmented energy spectra. Thus, while states located at the edges or the band centers of the main subbands exhibit a distinctive self-similar spatial structure, most of the remaining states do not show any specific pattern [48].

3.3. Extended States in General Fibonacci Lattices. In Section 2.1 we saw that, whereas both the on-site and standard transfer models are intrinsically noncommutative, one could always find the special energy value given by (40) satisfying $\left[\mathbf{R}_{A}, \mathbf{R}_{B}\right]=\mathbf{0}$ in general Fibonacci lattices. In this case the renormalized matrices given by (35) adopt the form:

$$
\begin{gathered}
\mathbf{R}_{B}=\left(\begin{array}{cc}
q^{2}-1 & -q \gamma \\
q \gamma^{-1} & -1
\end{array}\right), \\
\mathbf{R}_{A}=\left(\begin{array}{cc}
q\left(q^{2}-2\right) & \gamma\left(1-q^{2}\right) \\
\gamma^{-1}\left(q^{2}-1\right) & -q
\end{array}\right),
\end{gathered}
$$

where

$$
q \equiv \frac{2 \epsilon \gamma}{1-\gamma^{2}}
$$

Since $q \neq 0$ in the general Fibonacci lattice (the choice $\epsilon=$ 0 leads to the induced transfer model), $\mathbf{R}_{B}$ cannot adopt a diagonal form, but $\mathbf{R}_{A}$ becomes diagonal for $q= \pm 1$. In that case, $\mathbf{R}_{A}= \pm \mathbf{I}$ and the global transfer matrix reads

$$
\begin{aligned}
\mathscr{M}_{N}^{ \pm}\left(E_{*}\right) & =( \pm 1)^{n_{A}} \mathbf{R}_{B}^{n_{B}} \\
& =( \pm 1)^{n_{A}}\left(\begin{array}{cc}
U_{n_{B-2}}\left(-\frac{1}{2}\right) & \mp \gamma U_{n_{B}-1}\left(-\frac{1}{2}\right) \\
\mp \gamma^{-1} U_{n_{B}-1}\left(-\frac{1}{2}\right) & U_{n_{B}}\left(-\frac{1}{2}\right)
\end{array}\right),
\end{aligned}
$$

where we have made use of the Cayley-Hamilton theorem to evaluate the required power matrix.

Quite interestingly the matrices given by (60) can be expressed as powers of a common matrix themselves; namely, $\mathbf{R}_{B}=\mathbf{R}^{2}$ and $\mathbf{R}_{A}=\mathbf{R}^{3}$, where

$$
\mathbf{R}=\left(\begin{array}{cc}
q & -\gamma \\
\gamma^{-1} & 0
\end{array}\right)
$$

is a unimodular matrix [83]. In this way, making use of the Cayley-Hamilton theorem, the global transfer matrices corresponding to the energies given by (40) can be readily expressed in the closed form:

$$
\mathscr{M}_{N}\left(E_{*}\right)=\mathbf{R}^{N}=\left(\begin{array}{cc}
U_{N} & -\gamma U_{N-1} \\
\gamma^{-1} U_{N-1} & -U_{N-2}
\end{array}\right),
$$

where $U_{k}(x)$ are Chebyshev polynomials of the second kind and

$$
x=\frac{q}{2}=\frac{1}{2} \sqrt{E_{*}^{2}-\epsilon^{2}} \equiv \cos \phi .
$$

Taking into account the relationship $U_{n}-U_{n-2}=2 T_{n}$ between Chebyshev polynomials of the first and second kinds, from (64), one gets $\operatorname{tr}\left[\mathscr{M}_{N}\left(E_{*}\right)\right]=2 \cos (N \phi)$. Consequently, we can ensure that energies determined by (40) belong to the spectrum in the QP limit $(N \rightarrow \infty)$.

The charge distribution shown in Figure 20(a) corresponds to a state of energy $E_{1}^{*}=-1.25$ in a general Fibonacci chain with $N=F_{16}=1597$ sites and lattice parameters $\gamma=2$ and $\epsilon=0.75$. Figure 20(b) shows the charge distribution corresponding to a state of energy $E_{2}^{*}=-5 / 6$ in a general Fibonacci lattice of the same length and model parameters $\gamma=2$ and $\epsilon=0.5$. The overall behavior of the wave functions amplitudes clearly indicates their extended character. Indeed, the presence of identity matrices in the product defining the global transfer matrix favours the existence of extended states spreading relatively homogeneously throughout the lattice. At this point it is worthwhile to mention that, despite its appearance, this wave function is nonperiodic: the sequence of values taken by the wave function amplitude is arranged according to a $\mathrm{QP}$ sequence.

An illustrative example of the energy spectra of general Fibonacci chains for different values of the $\gamma$ model parameter is shown in Figure 21. Its characteristic fragmentation scheme is clearly visible. One appreciates that the extended states given by (40) are located in the densest regions of the phase diagram. The corresponding band edge states are obtained from the condition $\left|\operatorname{tr}\left[\mathscr{M}_{N}\left(E_{*}\right)\right]\right|=2$ and, for an arbitrary $N$ value, they are given by the condition $\phi=0, \pi \Rightarrow$ $x= \pm 1$. This condition is satisfied by the model parameters combinations $\epsilon \gamma= \pm\left(1-\gamma^{2}\right)$, respectively. For the argument values $x= \pm 1$ the Chebyshev polynomials considerably simplify to the form $U_{n}(1)=n+1$ and $U_{n}(-1)=(-1)^{n}(n+1)$, and the global transfer matrices can be expressed as

$$
\begin{gathered}
\mathscr{M}_{N}\left(E_{*}^{+}\right)=\left(\begin{array}{cc}
N+1 & -\gamma N \\
\gamma^{-1} N & 1-N
\end{array}\right), \\
\mathscr{M}_{N}\left(E_{*}^{-}\right)=(-1)^{N}\left(\begin{array}{cc}
N+1 & \gamma N \\
-\gamma^{-1} N & 1-N
\end{array}\right),
\end{gathered}
$$

where $E_{*}^{+}\left(E_{*}^{-}\right)$denote the eigenstate for the top (bottom) band edge, respectively. In both cases the wave function amplitudes grow linearly with the lattice site. 


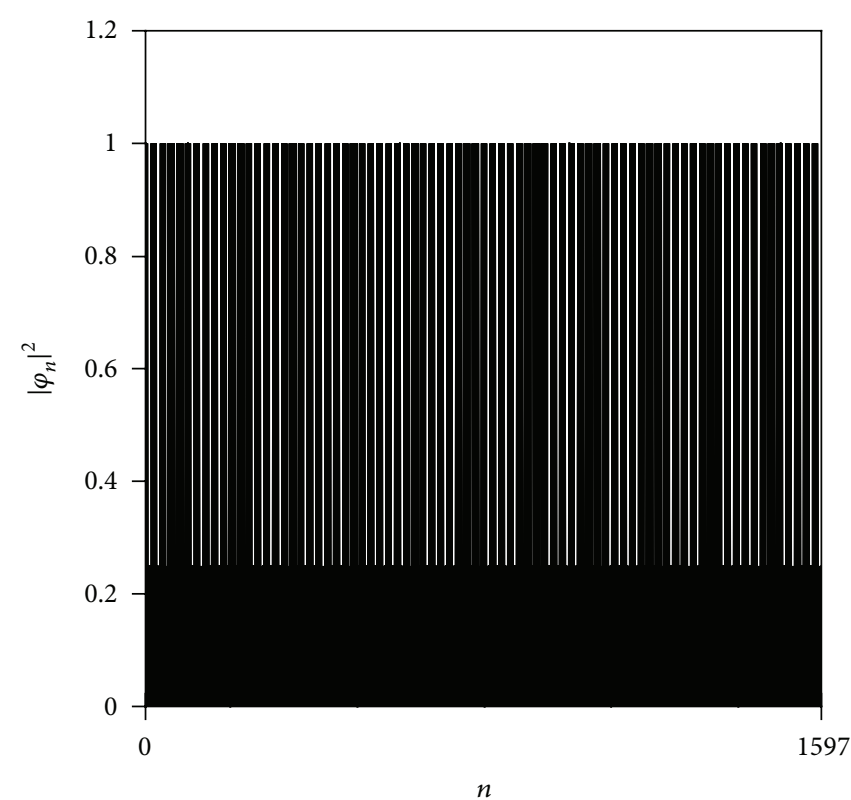

(a)

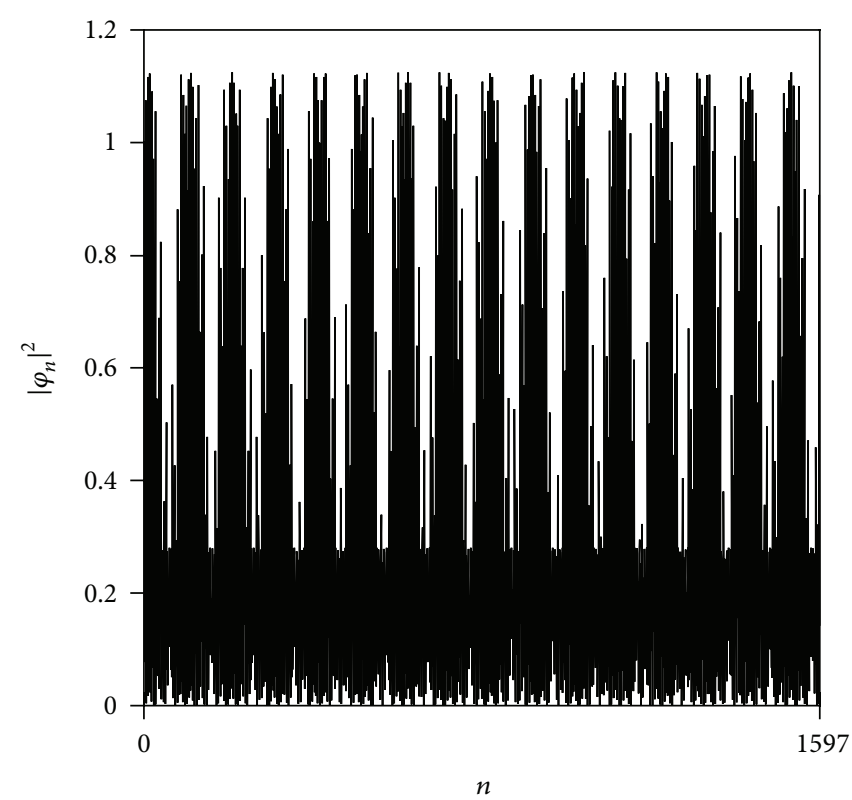

(b)

FIGURE 20: Electronic charge distribution in general Fibonacci lattices with $N=F_{16}$ and (a) $\gamma=2, \epsilon=0.75$, and $E_{1}^{*}=-5 / 4$ and (b) $\gamma=2$, $\epsilon=0.5$, and $E_{2}^{*}=-5 / 6$. The wave function amplitudes have been calculated with the aid of the matrix formalism making use of the initial conditions $\varphi_{0}=0$ and $\varphi_{1}=1$ (from [49], with permission from Maciá and Domínguez-Adame ( 1996 by the American Physical Society).

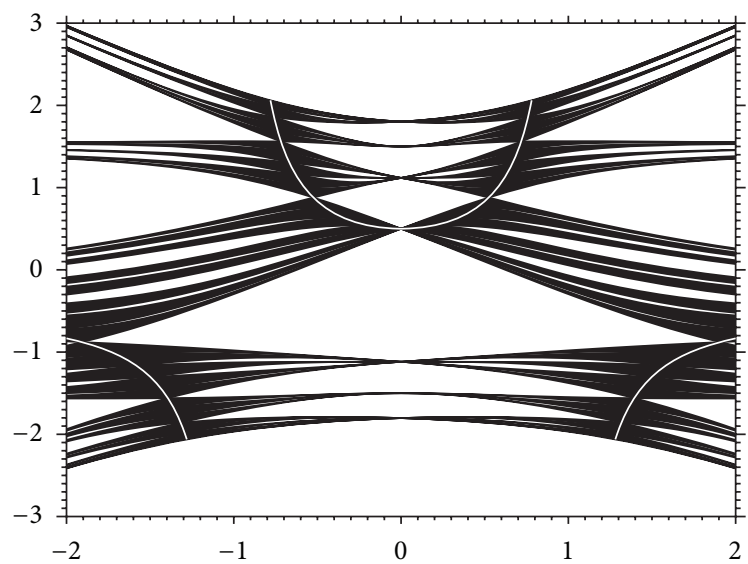

FIGURE 21: Phase diagram ( $\gamma$ in abscissas and $E$ in ordinates) for a general Fibonacci chain with $N=34$ and $\epsilon=0.5$. The energies corresponding to transparent wave functions are marked with a thin white line (courtesy of Roland Ketzmerick, from [46], with permission from CRC Taylor \& Francis group).

\section{The Role of Critical States in Transport Properties}

4.1. Signatures of Quasiperiodicity. As we saw in Section 1.2, the nature of a wave function was at first established by attending to its spatial structure. This criterion has proved useful as far as it was possible to assign a precise relationship between such a spatial structure and its related transport properties. Thus, the amplitudes distribution of a Bloch extended state fits a periodically modulated pattern, whereas for a localized state this distribution decays exponentially, according to a well-defined localization length. Therefore, for both localized and extended states, the corresponding wave functions admit a global description in terms of an envelope function, which is characterized by a specific spatial parameter, such as the localization length or the period, respectively. On the contrary, a global description of the wave function in terms of an envelope function is no longer possible, in general, for the eigenstates belonging to aperiodic systems due to the fluctuating structure shown by their amplitude distributions.

As we have previously mentioned, from a physical viewpoint electronic states can be more appropriately classified according to their transport properties, which are of course closely related to the spatial distribution of the wave function amplitudes as well. One can then reasonably expect that the rich structure displayed by most eigenstates in QPS should be reflected, to some extent, in their related transport properties. In order to explore this issue, Sutherland and Kohmoto studied the electrical resistance of the six-cycle and twocycle states located at the center and at the band-edges of the allowed subbands in a binary Fibonacci alloy [84]. To this end, they made use of the dimensionless Landauer formula:

$$
\rho_{N}(E)=\frac{1}{4}\left(M_{11}^{2}+M_{12}^{2}+M_{21}^{2}+M_{22}^{2}-2\right)
$$

where $M_{i j}(E)$ are the elements of the global transfer matrix. They found that the Landauer resistance is bounded above by a quantity which scales according to a power-law as a function of the length of the system: $\rho_{N}(E) \leq \rho_{0} N^{\xi}$, with 
$\xi=\ln t / \ln \tau^{Q}$, where $\tau$ is the golden mean and $Q=6$ $(Q=2)$ for six-cycle (two-cycle) eigenstates. On the basis of these exact results, valid for a subset of eigenstates, it was conjectured that the resistance as a function of the chain size was bonded by a power of the sample length for all states belonging to the system spectrum.

This conjecture was subsequently rigorously confirmed by Iochum and Testard [28]. Therefore, the power-law scaling of the resistivity can be properly regarded as a fingerprint of critical states in Fibonacci lattices. On the other side, the plot of the Landauer resistance as a function of the system size shows self-similar fluctuations as a consequence of the highly fragmented structure of their energy spectrum, as it is illustrated in Figure 22.

Inspired by these results, the average transport properties in Fibonacci lattices were studied by considering the influence of a number $v$ of wave functions on the mean value of the Landauer resistance for a given system length, according to the average:

$$
\left\langle\rho_{N}\right\rangle \equiv \frac{1}{v} \sum_{i=1}^{v} \rho_{N}\left(E_{i}\right)
$$

where $E_{i}$ indicates the energy of the corresponding states. It was obtained that the average Landauer resistance also scales as $\left\langle\rho_{N}\right\rangle \sim N^{a}(a>0)$ [85]. The power-like increase of the resistance indicates that the electrical conductance vanishes in the limit $N \rightarrow \infty$. This trend qualitatively agrees with the large electrical resistivity values observed in most icosahedral quasicrystals containing transition metals $[46,86]$.

Another magnitude which has been profusely considered in the study of QPS is the transmission coefficient given by

$$
\begin{aligned}
& T_{N}(E) \\
& =\frac{4\left(\operatorname{det} \mathscr{M}_{N}\right)^{2} \sin ^{2} \kappa}{\left[M_{12}-M_{21}+\left(M_{11}-M_{22}\right) \cos \kappa\right]^{2}+\left(M_{11}+M_{22}\right)^{2} \sin ^{2} \kappa},
\end{aligned}
$$

where one assumes the aperiodic chain to be sandwiched between two periodic chains (playing the role of metallic contacts), each one with on-site energy $\varepsilon^{\prime}$ and transfer integral $t^{\prime}$. The dispersion relation of the contacts is then given by $E=\varepsilon^{\prime}+2 t^{\prime} \cos \kappa$. The transmission coefficient is a useful quantity to describe the transport efficiency in quantum systems. Nonetheless, $T_{N}(E)$ is usually difficult to be directly measured experimentally, though it is directly related to the dimensionless Landauer resistance through the expression:

$$
\rho_{N}(E)=\frac{1-T_{N}(E)}{T_{N}(E)} .
$$

Accordingly, for those states having a unity transmission coefficient, $T_{N}(E)=1$, usually referred to as transparent states, one expects a ballistic transport throughout the lattice. Certainly, this condition is fulfilled by extended Bloch functions describing extended states. But, what can be said about the possible existence of transparent states in fractal and QPS?
4.2. Transparent Extended States in Aperiodic Systems. Let us now consider a class of wave functions belonging to either $\mathrm{QP}$ or fractal systems, which satisfy the condition $T_{N}(E)=1$, so that they can be regarded as extended from a physical point of view, though their wave function amplitudes distribution also exhibits characteristic QP features. This result prompts one to properly widen the notion of transparent wave function to include electronic states which are not Bloch functions, thereby clarifying the precise manner in which the QP structure of these wave functions influences their transport properties.

Indeed, from a detailed numerical study of the triadic Cantor lattice it was found that the transmission coefficient becomes equal to unity for many energy eigenvalues corresponding to extended eigenfunctions of the system, as it is illustrated in Figure 23. However, it should be mentioned that there are also values of the transmission coefficient which lie between zero and one. For example, the transmission coefficient corresponding to the energy $E=0$ in the on-site model with $V_{A}=0, V_{B}=-1, t=1, \epsilon^{\prime}=0$, and $t^{\prime}=1$ reads

$$
T_{N}(0)=\left[1+\frac{1}{3} \sin ^{2}\left(4(k-1) \frac{\pi}{3}\right)\right]^{-1},
$$

for $k>1$, where the $k$ is the fractal generation order determining the chain length, $N=3^{k}$. Accordingly, when $k-1$ is a multiple of 3 the state becomes transparent, elsewhere one gets $T_{N}(0)=4 / 5=0.8$, as it is the case of the eigenstate shown in Figure 17 for a lattice with $N=3^{5}$ sites. Therefore, among the extended wave functions belonging to the triadic Cantor lattice spectrum energy one can find both transparent and nontransparent states, depending upon the adopted $N$ value [60].

A similar result is obtained when considering the selfsimilar state corresponding to $E=0$ in the standard transfer model of the Fibonacci binary alloy (Figure 18). As we saw in Section 3.2, its global transfer matrix exhibits a six-cycle property so that, depending on the $N$ value, there exist five possible types of resulting $\mathscr{M}_{N}(0)$ matrices, as prescribed by (56). By plugging the corresponding matrix elements $M_{i j}$ into (69) one gets $T_{N^{*}}(E)=1$ when the transfer matrix adopts the simple form given by $\mathscr{M}_{1}(0)$, whereas one has

$$
T_{N}(0)=\frac{4 t_{B}^{2}}{\left(1+t_{B}^{2}\right)^{2}},
$$

elsewhere. Since this expression is less than unity for all transfer integral values different from the trivial periodic case $t_{B}^{2}=1,{ }^{22}$ one concludes that $T_{N}(0)<1$ for a large number of $N=F_{j}$ values. In fact, the state shown in Figure 18 $\left(N=17711=F_{21}\right)$ is not a transparent one but yields $T_{N}(0)=16 / 25=0.64$. At the same time, there also exists a significant number of transparent states, corresponding to the chain lengths obeying the series $N^{*}=F_{4+3 k}, k=0,1,2, \ldots$. A completely analogous result is obtained for the state $E=0$ in the induced transfer model of the binary Fibonacci alloy. In fact, making use of the global transfer matrix given by (39) in (69) one gets $T_{N}(0)=1$ or $T_{N}(0)=4\left(\gamma+\gamma^{-1}\right)^{-2} \sin ^{2} \kappa$, depending upon whether $N$ is even or odd, respectively. Thus, 


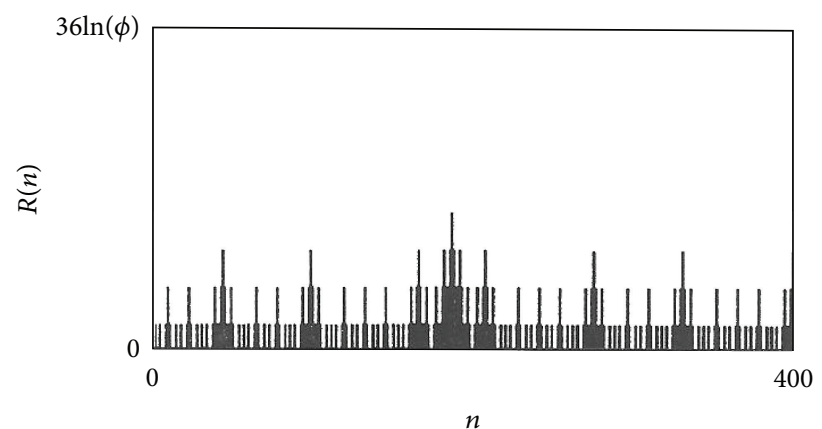

(a)

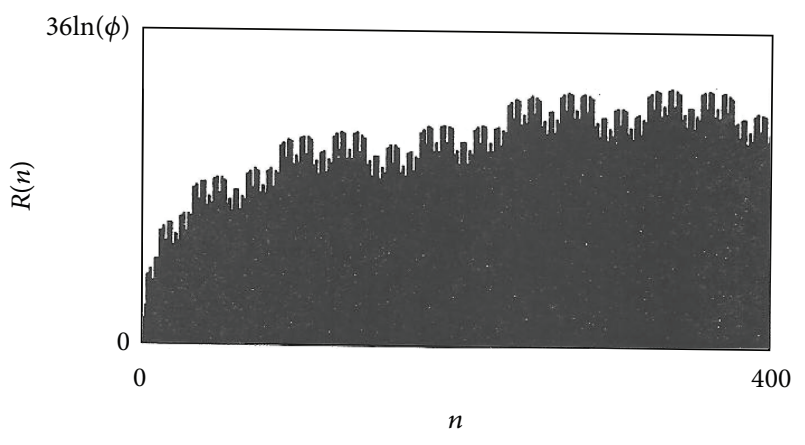

(b)

FIGURE 22: The quantity $R(n)=\ln \left(2 \rho_{n}-1\right)$ is shown as a function of the lattice site $n$ for (a) six-cycle and (b) two-cycle states (from [84], with permission from Sutherland and Kohmoto (c) 1987 by the American Physical Society).

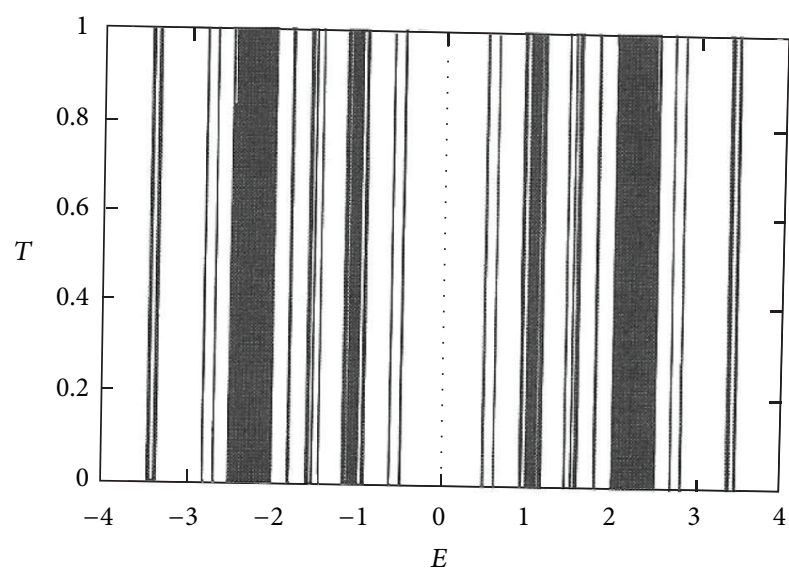

Figure 23: Transmission coefficient as a function of the electron energy for a 16th generation triadic Cantor lattice. All the on-site energies are set equal to zero and $t_{L}=1, t_{S}=2, \epsilon^{\prime}=0$, and $t^{\prime}=1$ (from [60], with permission from Elsevier).

in this case we obtain a remarkable $N$ parity effect in the resulting transport properties.

The properties of electronic states are even more interesting in general Fibonacci lattices when one considers the subset of the energy spectrum formed by those eigenstates satisfying (40). In this case, by plugging the global transfer matrix elements given by (64) into (69) one obtains [49]

$$
T_{N}\left(E_{*}\right)=\left[1+\frac{\left(1-\gamma^{2}\right)^{2}}{\left(4-E_{*}^{2}\right) \gamma^{2}} \sin ^{2}(N \phi)\right]^{-1} .
$$

Two important conclusions can be drawn from this expression. In the first place, the transmission coefficient is always bounded below (i.e., $T_{N}\left(E_{*}\right) \neq 0$ ) for any lattice length, which proves the truly extended character of the related states. In the second place, since the factor multiplying the sine in the denominator of expression (73) only vanishes in the trivial (periodic) case $\gamma=1$, the critical states we are considering do not verify, in general, the transparency condition $T_{N}\left(E_{*}\right)=1$ in the QP limit.
For the sake of illustration, consider the extended states shown in Figure 20. At first sight, by comparing both figures, one may be tempted to think that the transmission coefficient corresponding to the wave function plotted in Figure 20(a) should be higher than that corresponding to the wave function shown in Figure 20(b), because the charge distribution of the former along the system is more homogeneous than that corresponding to the latter. Actually, however, making use of expression (73), one finds $T\left(E_{1}^{*}\right)=0.5909 \cdots$ and $T\left(E_{2}^{*}\right)=0.7425 \cdots$, which is just the opposite case. Therefore, despite the homogeneous distribution of their amplitudes, these estates are not transparent at all.

However, according to (73) it is also possible to find states satisfying the transparency condition in general Fibonacci lattices whose lengths satisfy the relationship $N \phi=k \pi, k=$ $1,2, \ldots$, which, after (65), implies

$$
E_{*}(k)= \pm \sqrt{\epsilon^{2}+4 \cos ^{2}\left(\frac{k \pi}{N}\right)} \text {. }
$$

In this way, these transparent states can be classified according to a well-defined scheme determined by the integer $k$. By combining (40) and (74) we obtain the following relationship for the values of the model parameters satisfying the transparency condition:

$$
\epsilon= \pm \frac{1-\gamma^{2}}{\gamma} \cos \left(\frac{k \pi}{N}\right)
$$

Quite remarkably, it was reported that when one chooses the model parameters in such a way that they satisfy (75), with $k$ being a divisor of $N$ (i.e., $N / k=p \in \mathbb{N}$ ), one will always get a periodically modulated wave function which propagates ballistically through the system [87]. The periodicity of the long scale modulation is a direct consequence of the divisibility properties of Fibonacci numbers; namely, $F_{k}$ is a divisor of $F_{p k}$ for all $p>0$ [88]. For a given system size, the smaller $k$ is, the more periods the envelope of the wave functions has, and the period of this envelope is exactly $N / k$. Thus, the wave function amplitudes distribution becomes progressively more homogeneous as the value of 
$k$ gets smaller, as it is illustrated in the different panels of Figure 24. In that case, the wave functions exhibit a remarkable spatial distribution, where the QP component (characteristic of short spatial scales) is nicely modulated by a long scale periodic component.

On the other hand, from (73) we see that among the possible transparent states there exists a subset satisfying the condition $\phi=0(\phi=\pi)$, so that these states are transparent for any $N$ value. As we discussed in Section 3.3, these particular states correspond to the band-edges of the energy spectrum, and they satisfy the condition $\epsilon \gamma= \pm(1-$ $\gamma^{2}$ ). By comparing with (75) we realize that these band-edge states correspond to the label $k=0$ and $k=N$ (see Figure 24(a)).

Subsequent numerical studies of the energy spectrum of general Fibonacci lattices have shown that a significant number of electronic states exhibiting transmission coefficients close to unity are located around the transparent states given by (40) [89]. An illustrative example is shown in Figure 25 for an energy value close to the resonance energy $E_{*}=-13 / 50=$ -0.26 . By inspecting this plot we notice the existence of two different superimposed structures. In fact, a periodic-like, long-range oscillation with a typical wavelength of about $N$ sites is observed to modulate a QP series of short-range minor fluctuations of the wave function amplitude, typically spreading over a few lattice sites. This qualitative description receives a quantitative support from the study of its Fourier transform, showing two major components in the Fourier spectrum corresponding to the low and high frequency regions, respectively. This result suggests that these critical states behave in a way quite similar to conventional extended states from a physical viewpoint, albeit they can not be rigorously described in terms of Bloch functions. To further analyze this important issue the study of the ac conductivity at zero temperature is very convenient, since it is very sensitive to the distribution nature of eigenvalues and the localization properties of the wave function close to the Fermi energy. In this way, by comparing the ac conductivities corresponding to both periodic and general Fibonacci lattices it was concluded that the value of the ac conductivity takes on systematically smaller values in the Fibonacci case, due to the fact that the ac conductivity involves the contribution of nontransparent states within an interval of $\hbar \omega$ around the Fermi level in this case $[90,91]$.

In summary, although when considering Bloch functions in periodic systems the notion of extended wave functions coincides with transparent ones, this is no longer true in the case of fractal and QPS. In particular, for general Fibonacci systems in which both diagonal and off-diagonal QP order are present in their model Hamiltonian, we have critical states which are not localized (i.e., $T_{N}\left(E_{*}\right) \neq 0 \forall N$, when $N \rightarrow$ $\infty)$. For finite Fibonacci chains one can find transparent states exhibiting a physical behavior completely analogous to that corresponding to usual Bloch states in periodic systems (i.e., $T_{N}\left(E_{*}\right)=1$ ) for a given choice of the model parameters, prescribed by (75). There exists a second class of critical states, those located close to the transparent ones, which are not strictly transparent (i.e., $T_{N}(E) \lesssim 1$ ) but exhibit transmission coefficient values very close to unity. Finally, the remaining states in the spectrum show a broad diversity of possible values of the transmission coefficient (i.e., $0<$ $\left.T_{N}(E) \ll 1\right)$, in agreement with the earlier view of critical states as intermediate between periodic Bloch wave functions $\left(T_{N}(E)=1\right)$ and localized states $\left(T_{N}(E)=0\right)$.

4.3. Electronic States in Koch Fractal Lattices. To conclude, let us consider the transport properties of electronic states in the Koch lattice. This lattice is topologically more complex than any of the previously considered lattices, mainly due to the fact that it has sites with different coordination numbers (see Figure 15), whereas all sites in Fibonacci, triadic Cantor and Vicsek fractal lattices are simply twofold coordinated. This higher topological complexity naturally translates to the $\mathbf{P}$ and $\mathbf{Q}$ renormalized matrices as well as to their commutator, which adopt a more complicated form than those obtained for the renormalized matrices in Fibonacci binary alloys, the triadic Cantor lattice, and the Vicsek fractal. Accordingly, the commutator (51) vanishes in four different cases. (i) The choice $\lambda=0$ reduces the original Koch lattice to a trivial periodic chain, so that all the allowed states, $-2 \leq E \leq 2$, are extended. (ii) The center of the energy spectrum, $E=0$, corresponds to an extended state [66]. (iii) $E= \pm \sqrt{2}$. (iv) The family of states $E=-2 / \lambda$. For these energies the global transfer matrix of the system, $M_{k}=\mathbf{P}^{n_{A}} \mathbf{Q}^{n_{B}}$, with $n_{A}=$ $4^{k-1}+1$ and $n_{B}=4^{k-2}+1$, can be explicitly evaluated. From the knowledge of $\mathscr{M}_{k}$ the condition for the considered energy value to be in the spectrum, $\left|\operatorname{tr}\left[\mathscr{M}_{k}\right]\right| \leq 2$, can be readily checked and, afterwards, its transmission coefficient can be explicitly determined.

Let us consider, in the first place, the energies $E= \pm \sqrt{2}$. In this case we get

$$
\operatorname{tr}\left[\mathscr{M}_{k}\right]=-\frac{1}{(1 \pm \sqrt{2} \lambda)^{n_{A}-n_{B}}}-(1 \pm \sqrt{2} \lambda)^{n_{A}-n_{B}} .
$$

A detailed study of the condition $\left|\operatorname{tr}\left[\mathscr{M}_{k}\right]\right| \leq 2$ in (76) indicates that the only allowed states correspond to $\lambda=\mp \sqrt{2}$, for which $\operatorname{tr} \mathscr{M}_{k}=-2$. Consequently, these states are just two particular cases of the more general family (iv) which we will discuss next.

By taking $E=-2 / \lambda$ we get $\mathbf{Q}=-\mathbf{I}$, where $\mathbf{I}$ is the identity matrix, so that $\mathscr{M}_{k}=-\mathbf{P}^{n_{A}}$. Making use of the CayleyHamilton theorem, the global transfer matrix corresponding to the energies $E=-2 / \lambda$ can be expressed in terms of Chebyshev polynomials of the second kind, $U_{n}(x)$, with $x \equiv$ $\cos \phi=-1+8 \lambda^{-2}-8 \lambda^{-4}$, in the closed form [55]:

$$
\mathscr{M}_{k}=\left(\begin{array}{cc}
U_{n_{A}-1}-U_{n_{A}} & -q U_{n_{A}-1} \\
q U_{n_{A}-1} & U_{n_{A}-2}-U_{n_{A}-1}
\end{array}\right),
$$

where $q \equiv 2\left(\lambda^{2}-2\right) / \lambda^{2}$. From expression (77) we get $\operatorname{tr}\left[\mathscr{M}_{k}\right]=-2 \cos \left(n_{A} \phi\right)$, so that we can ensure that these energies belong to the spectrum in the fractal limit $(k \rightarrow \infty)$. 


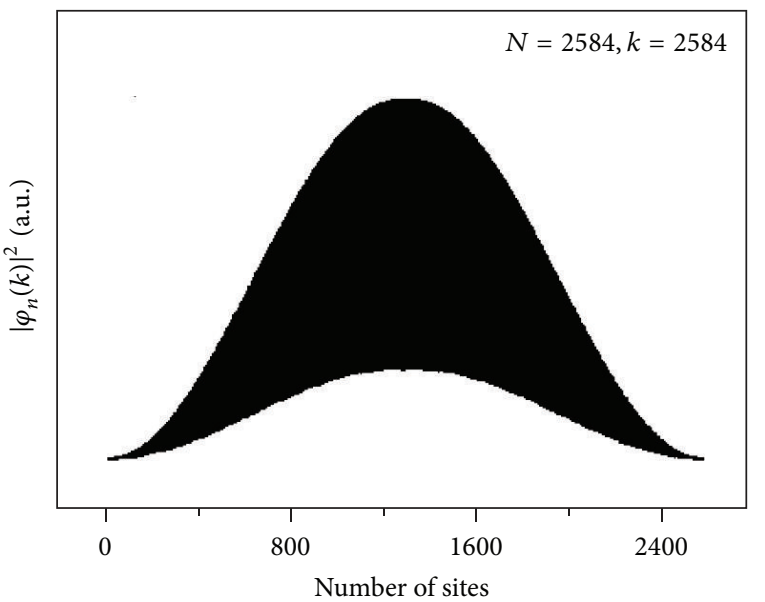

(a)

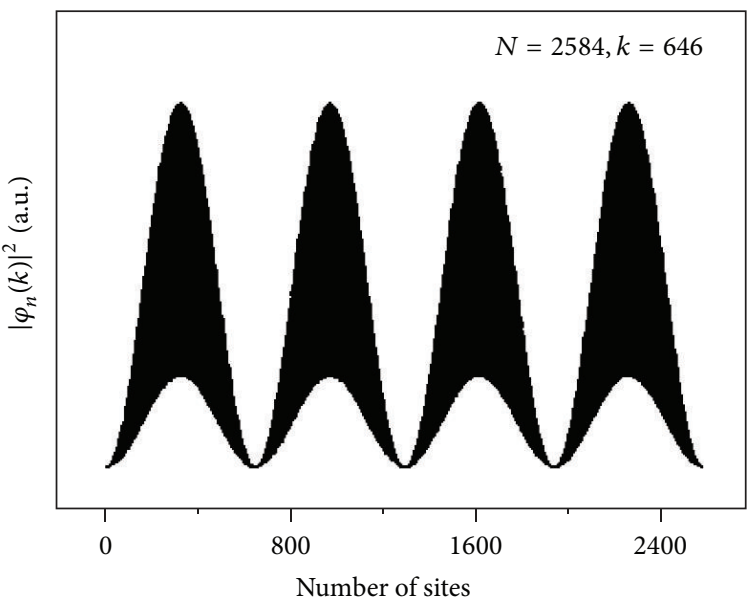

(c)

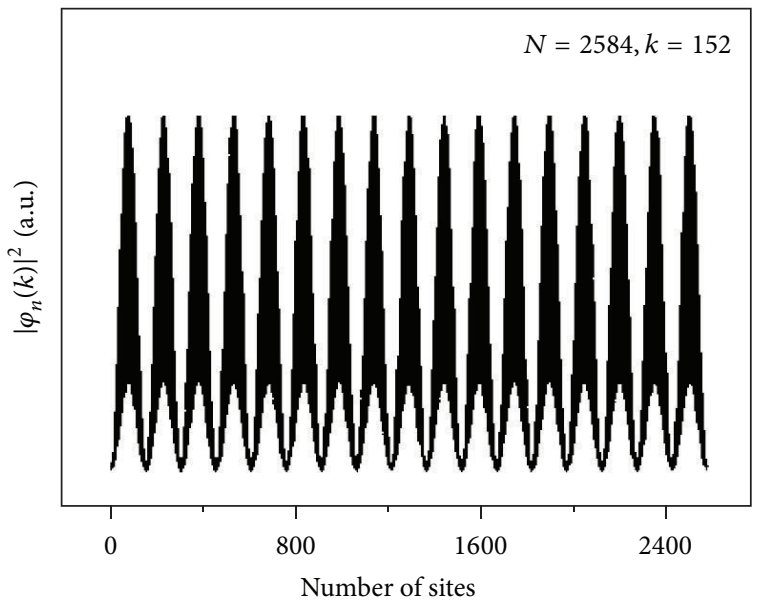

(e)

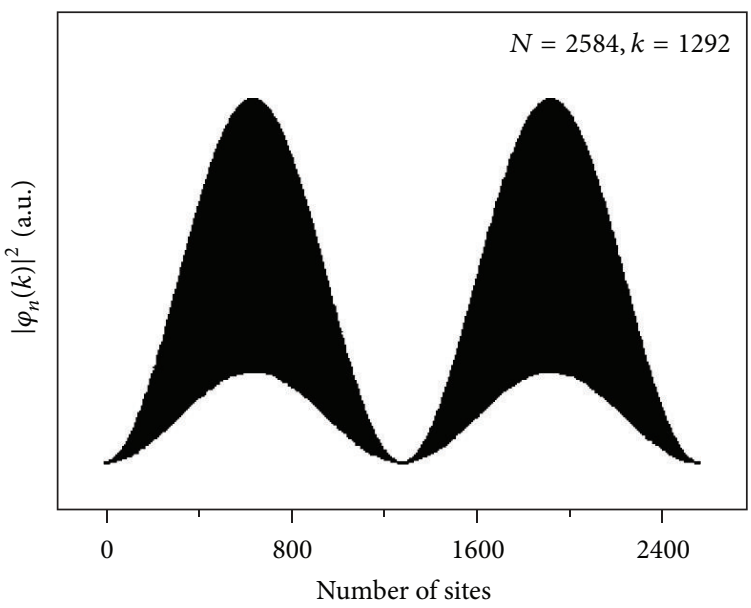

(b)

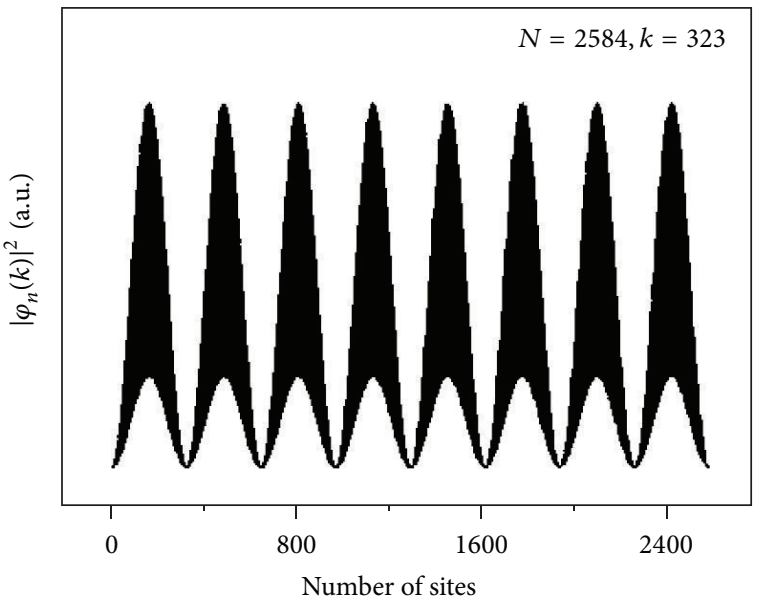

(d)

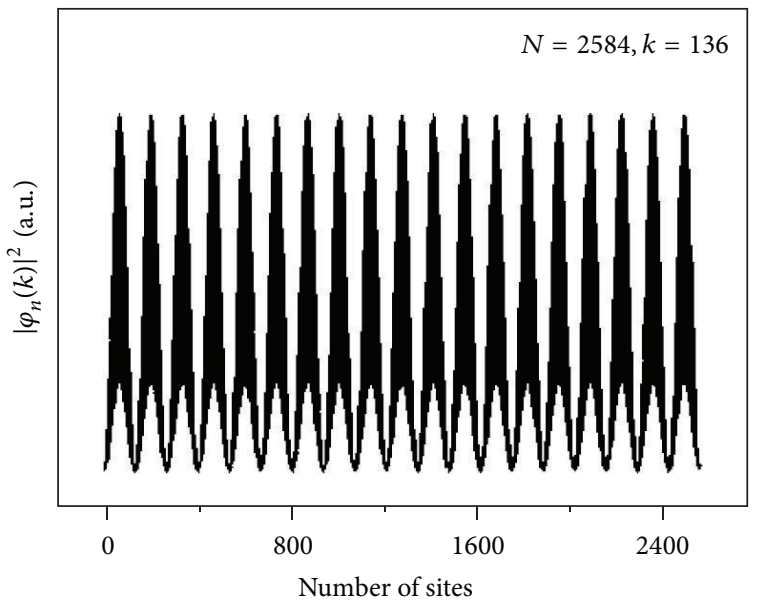

(f)

FIGURE 24: The wave function versus the site number for a Fibonacci lattice with $N=2584$ sites, $\gamma=2$, and $E_{*}(k)=-2.5 \cos (\pi / k)$ for different $k$ values (from [87], with permission from Huang and Gong $\odot 1998$ by the American Physical Society). 


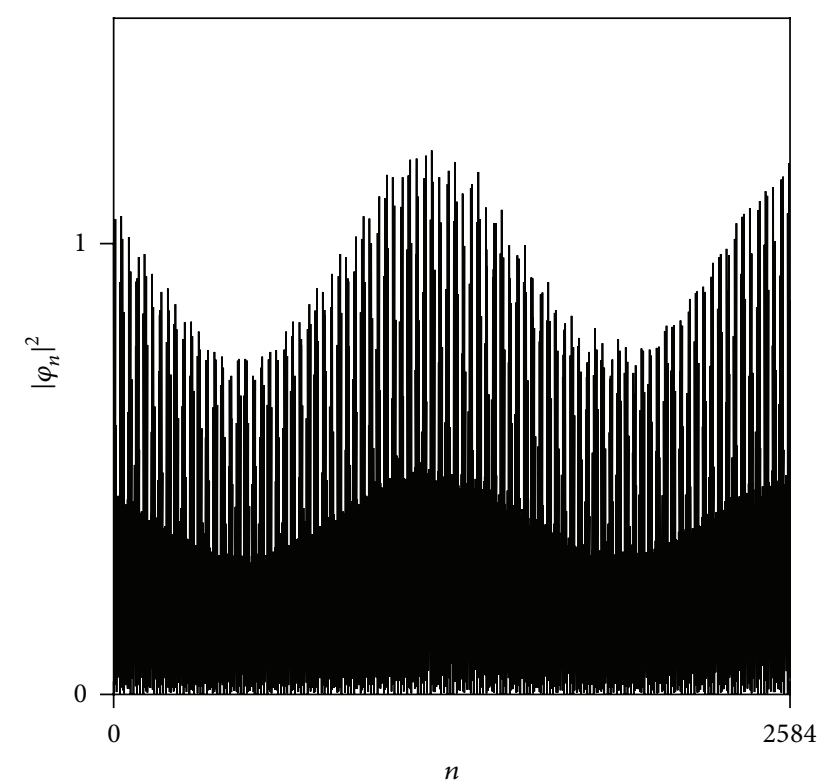

FIGURE 25: Electronic charge distribution corresponding to a periodic-like state with energy $E=-0.2601337$, for a general Fibonacci lattice with $N=F_{17}=2584, \gamma=1.5$, and $\epsilon=0.1$. Its transmission coefficient is $T_{N}(E) \simeq 1-10^{-15}$ (from [46], with permission from CRC Taylor \& Francis group).

The transmission coefficient at a given iteration stage reads [55]

$$
T_{k}(\lambda)=\frac{1}{1+\left[(\lambda(\lambda \pm 2) / 2(\lambda \pm 1)) \sin \left(n_{A} \phi\right)\right]^{2}},
$$

where the plus (minus) sign in the factor of $\sin \left(n_{A} \phi\right)$ corresponds to the choices $t^{\prime} \equiv 1$ and $t^{\prime} \equiv r=-1$, respectively, for the transfer integral of the periodic leads. From expression (78) we realize that the transmission coefficients corresponding to the family (iv) are always bounded below for any stage of the fractal growth process, which proves their extended nature in the fractal limit. In addition, the choices $\lambda= \pm 2(E=\mp 1)$ correspond to states which are transparent at every stage of the fractal growth process. A fact which ensures their transparent nature in the fractal limit as well. Furthermore, it is possible to find a number of cross-transfer integral values satisfying the transparency condition $T_{k}=1$ at certain stages of the fractal growth given by the condition $n_{A} \phi=p \pi, p \in \mathbb{N}$, which allows us to label the different transparent states at any given stage $k$, in terms of the integer p.

In Figure 26 we plot the transmission coefficient given by (78) at two successive stages $k=2$ and $k=3$, as a function of the cross-transfer value. In the first place, we note that the number of $\lambda$ values supporting transparent states, $\nu_{\lambda}$, progressively increases as the Koch curve evolves toward its fractal limit, according to the power law $\nu_{\lambda}=2\left(4^{k-1}+1\right)$. It is interesting to compare this figure with the number of sites, $N=4^{k}+1$, present, at the stage $k$, in the Koch lattice. Thus, we obtain $v_{\lambda}=(N+3) / 2$, indicating that the number of Koch lattices able to support transparent states increases linearly with the system size and, consequently, that the fractal growth favours the presence of transparent extended states in Koch lattices. In particular, we can state that, in the fractal limit, there exists an infinitely numerable set of crosstransfer integrals supporting transparent extended states in the Koch lattice.

Another general feature shown in Figure 26 is the presence of a broad plateau around $\lambda=2$ where the transmission coefficients take values significantly close to unity. In addition, as $\lambda$ separates from the plateau the local minima of the transmission coefficient, $T_{\min }$, take on progressively decreasing values which tend to zero in the limits $\lambda \rightarrow \infty$ and $\lambda \rightarrow 1$. This behavior suggests that the best transport properties in the family (iv) should be expected for those states located around the plateau.

Up to now we have shown that, as the Koch lattice approaches its fractal limit, an increasing number of crosstransfer integrals are able to support transparent states in the $E=-2 / \lambda$ branch of the phase diagram. But, for a given value of $\lambda$, how many of the related extended states at an arbitrary stage, say $k$, will prevail in the fractal limit $k \rightarrow \infty$ ? From a detailed analysis of expression (78) it was found that the considered states can be classified into two separate classes. In the first class we have those states which are transparent at any stage $k$. In the second class we find states whose transmission coefficient oscillates periodically between $T=1$ and a limited range of $T_{\min } \neq 1$ values depending on the value of the label integer $p$ and the fractal growth stage. Two general trends have been observed in this second class of extended states. First, the values of $T_{\min }$ are significantly lower for states corresponding to $p<0$ than for states corresponding to $p>0$. Second, at any given fractal stage, the values of $T_{\text {min }}$ are substantially higher for states associated with crosstransfer integral values close to the plateau than for states corresponding to the remaining allowed $\lambda$ values. We must note, however, that not all these almost transparent states are expected to transport in much the same manner, as suggested by the diversity observed in the values of $T_{\min }$.

In Figure 27 we provide a graphical account of the most relevant results obtained in this section. In this figure we show the phase diagram corresponding to the model Hamiltonian given by (48) at the first stage of the fractal process (shadowed landscape) along with two branches corresponding to the states belonging to the family $E=-2 / \lambda$ (thick black lines). In the way along each symmetrical branch we find three particular states whose coordinates are, respectively, given by $( \pm 1, \mp 2),( \pm \sqrt{2}, \mp \sqrt{2})$, and $( \pm 2, \mp 1)$. Three of them, corresponding to the choice $\lambda>0$, are indicated by full circles labelled A, B, and C in Figure 27. These states correspond to transparent states whose transmission coefficients equal unity at every $k$. The remaining states in the branches correspond to almost transparent states exhibiting an oscillating behavior in their transmission coefficients. By comparing Figures 26 and 27 we realize that the positions of the transparent states A-B-C allow us to define three different categories of almost transparent states according to their related transport properties. The first class (I) includes those states comprised between the state $\mathrm{A}$, at the border of the spectrum, 


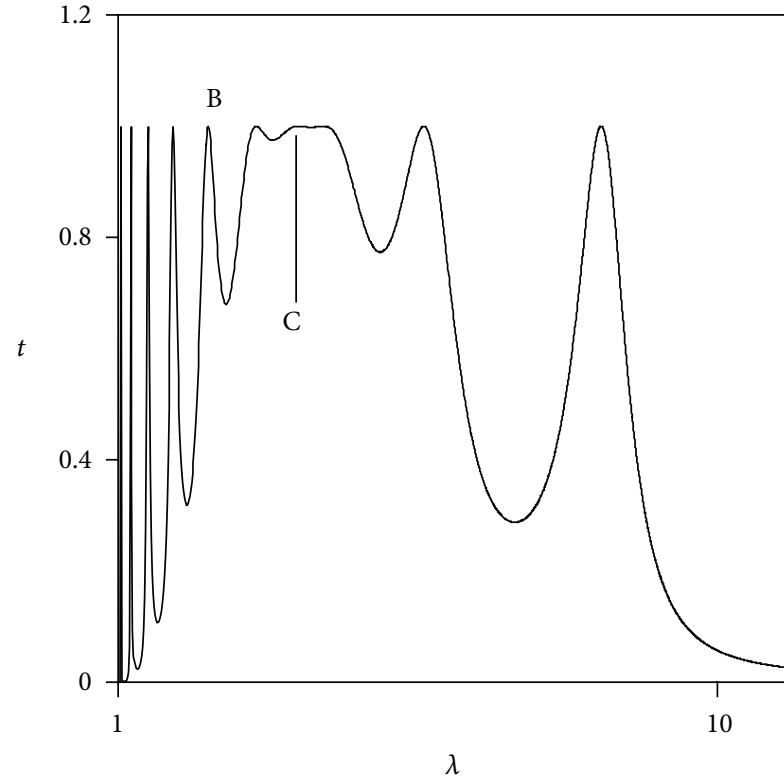

(a)

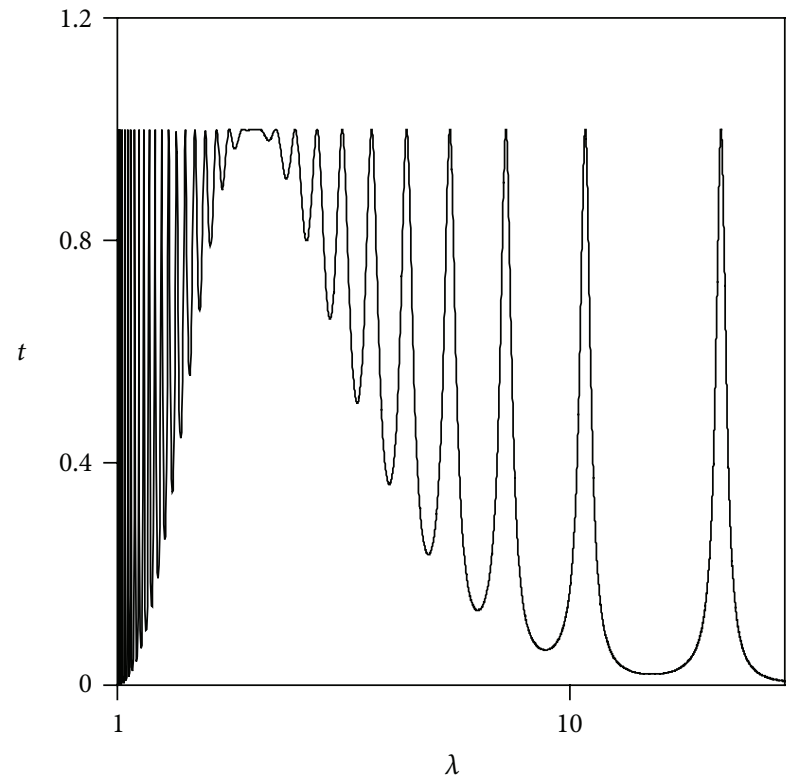

(b)

Figure 26: Transmission coefficient as a function of the cross-transfer integral at two different stages (a) $k=2$ and (b) $k=3$. Peaks are labelled from left to right starting with $p=-4$ in (a). Label B corresponds to $p=0$. Label C indicates the transparent state at $\lambda=2$ (from [55], with permission from Maciá (C) 1998 by the American Physical Society).

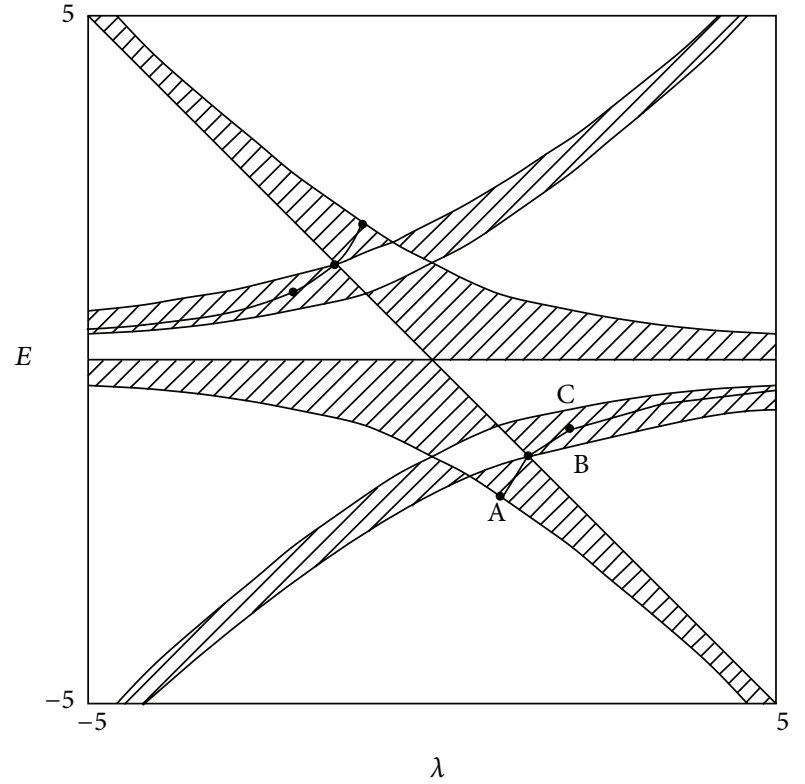

FIGURE 27: Phase diagram showing the Koch lattice spectrum at $k=$ 1 (shadowed areas) and the branches corresponding to the extended states family $E=-2 / \lambda$ (from [55], with permission from Maciá $\odot$ 1998 by the American Physical Society).

and the state B, located at a vertex point separating two broad regions of the phase diagram. The second class (II) includes those states comprised between the state $B$ and the state $C$ close to the plateau in the transmission coefficient around $\lambda=$ 2. Finally, the third class (III) comprises those states beyond the state C. The states exhibiting better transport properties belong to the classes II and III and correspond to those states grouping around the plateau near the state $C$ for which the values of $T_{\min }$ are very close to unity. As we move apart from state $\mathrm{C}$, the transport properties of the corresponding almost transparent states become progressively worse, particularly for the states belonging to the class III, for which values of $T_{\text {min }}$ as low as $10^{-3}$ can be found.

\section{Outlook and Perspectives}

In the preceding sections we have presented results obtained in the study of wave functions in fractal and QPS by mainly considering two complementary criteria, namely, their spatial structure and the value of their related transmission coefficients. In the case of periodic crystals or amorphous materials both criteria are simply related to each other. Thus, in periodic crystals we have Bloch states exhibiting a periodically extended spatial structure and $T_{N}(E)=1$ for any $N$ value. Therefore, the allowed states in the spectrum are both extended and transparent wave functions. On the other hand, uncorrelated random systems have exponentially localized wave functions with $T_{N}(E)=0$. Thus, all states in these systems are both localized and have vanishing transmission coefficients. These simple scenarios no longer apply to either fractal or QPS, having a richer class of wave functions exhibiting a plethora of amplitude distribution patterns. These patterns must be generally described in terms of multifractal analysis and exhibit a broad diversity of transmission coefficient values, spanning the full range $0<$ $T_{N}(E) \leq 1$. 
Two points should be highlighted regarding the transmission coefficient limiting values: (i) since $T_{N}(E) \neq 0$ in general, strictly localized states are not present in QPS; (ii) the very possibility of having $T_{N}(E)=1$ for some particular energy values indicates that the notion of transparent state must be widened to include eigenstates which are not Bloch functions at all.

Additionally, fractal systems generally possess an even richer variety of wave functions, stemming from their inherent combination of self-similarity (a property shared with QPS) and fractal dimensionality (manifested by the presence of sites with different coordination numbers along the chain).

The algebraic conditions $\mathbf{R}_{i}^{n}= \pm \mathbf{I}$ along with $\left[\mathbf{R}_{i}, \mathbf{R}_{j}\right]=\mathbf{0}$, where $\mathbf{R}_{i}$ denotes a suitable renormalized transfer matrix, allow one to properly pick up certain special energy values related to wave functions which exhibit quite homogeneous amplitude distributions, generally lacking distinctive selfsimilar features. The absence of self-similar arrangements of peaks can be traced back to the fact that, for these energies, the global transfer matrix for the original fractal or QP lattice can be expressed in terms of an effective periodic chain (e.g., triadic Cantor lattice) or two effective periodic chains (e.g., general Fibonacci lattices) upon appropriate decimation/renormalization of the original chains. In both cases, such a possibility naturally leads to the presence of periodic-like distributions of the wave functions amplitudes.

By keeping in mind the main results reviewed in this work concerning the spatial structure of the wave functions belonging to fractal and QPS, it could be concluded that the recent trend of abandoning the former use of "critical wave function" to generically refer to all eigenstates belonging to singular continuous spectra should be encouraged [92]. In fact, whereas the electronic states found in the Aubry-André self-dual model can be properly regarded as being strictly critical ones (in the sense that they undergo a metal-insulator critical transition) this criterion no longer holds for the states found in both fractal and QPS, albeit all of them display multifractal properties. In our opinion, the fundamental property in order to properly classify the nature of states in aperiodic systems must focus on their related transport properties rather than on the wave function amplitudes distribution patterns. The very existence of transparent (ballistic propagation) states in these systems provides conclusive evidence on the unsuitability of the dichotomist view in terms of Bloch and exponentially localized states in order to account for the electronic transport properties in aperiodic systems. Accordingly, we would like to tentatively propose the use of the term singular wave functions to generically refer to the broad family of eigenstates belonging to aperiodic systems exhibiting singular continuous energy spectra.

\section{Conflict of Interests}

The author declares that there is no conflict of interests regarding the publication of this paper.

\section{Endnotes}

1. The theory of these functions was developed by Harald Bohr (1887-1951), brother of the well-known physicist Niels Bohr [93].

2. As an illustrative counter example we can consider the function:

$$
f(x)=\lim _{N \rightarrow \infty} \sum_{n=1}^{N} \frac{1}{n !} \sin \left(\frac{x}{2^{n}}\right),
$$

which is AP but not QP, since its Fourier spectrum does not have a finite basis.

3. Though exponentially localized decaying states are considered as representative of most localization processes in solid state physics one must keep in mind that other possible mathematical functions are also possible to this end.

4. The tight-binding approximation considers that both the potential and the electronic wave functions are sharply peaked on the atomic sites.

5. We note that (9) is invariant under the simultaneous transformation:

$\psi_{n} \longrightarrow(-1)^{n} \psi_{n}, \quad V_{n} \longrightarrow-V_{n}, \quad E \longrightarrow-E$.

This property shows that the spectra corresponding to opposite values of the potential are mirror images of each other with respect to the center of the spectrum.

6. For the sake of simplicity one can adopt units such that $\hbar=2 m$ without loss of generality.

7. Note that all of the transfer integrals take on the same value.

8. The existence of exponentially localized wave functions has been recently reported from numerical studies of (10) with the bichromatic potential $V(x)=2 \lambda_{1} \cos x+$ $2 \lambda_{2} \cos \alpha x$ with rational $\alpha=p / q$ corresponding to certain specific energy values $\left(E>2\left(\lambda_{1}+\lambda_{2}\right)\right)$. This system then suggests the possible presence of a purepoint spectral component not restricted to low energy values in certain periodic systems [44].

9. This equation is also referred to as Harper's equation (see Section 1.6) or the almost Mathieu operator, which results from the discretization of the classical Mathieu equation:

$$
\frac{d^{2} f}{d x^{2}}+\left(b_{0}+b_{1} \cos x\right) f
$$

where $b_{0}$ and $b_{1}$ are real constants.

10. The details of the crossover between localized and extended regimes have been studied in detail for some special values of irrational $\alpha$ values only, so that one may ask whether these particular $\alpha$ values are representative enough.

11. That is, $\alpha=\lim _{m \rightarrow \infty}\left(p_{m} / q_{m}\right)$, where $p_{m}$ and $q_{m}$ are coprime integers. 
12. By comparing (14) and (20) we realize that in Harper's equation the potential strength maximum value is constrained to the value $\lambda=2$ from the onset, whereas in the Aubry-André equation this potential strength maximum is one of the free parameters of the model.

13. Note that this discontinuous character excludes the possibility of an absolutely continuous spectral measure.

14. Quite interestingly, this description written in the prefractal era uses the term "clustering" instead of "splitting" in order to describe the energy spectrum fragmented pattern. This semantics seems to be more appropriate when considering the physical origin of the hierarchical patterns in terms of resonance effects involving a series of neighboring building blocks interspersed through the lattice.

15. The choice of binary lattices composed of just two different types of atoms is just a matter of mathematical simplicity, though one should keep in mind that all of the thermodynamically stable QCs synthesized during the period 1987-2000 were ternary compounds. In 2000 the discovery of the first binary QC was reported (belonging to the (Cd, Ca) Yb system), and its atomic structure was solved in 2007 [7].

16. To the best of our knowledge, this state was first studied by Kumar [50], who apparently confused it with the $E=$ 0 eigenstate of the Fibonacci standard transfer model shown in Figure 13(d).

17. According to the Cayley-Hamilton theorem any $n \times n$ square matrix over the real or complex field is a root of its own characteristic polynomial [94]. Making use of this property, if $\mathbf{M}$ belongs to the $\operatorname{Sl}(2, \mathbb{R})$ group, one can readily obtain by induction the expression:

$$
\mathbf{M}^{N}=U_{N-1}(z) \mathbf{M}-U_{N-2}(z) \mathbf{I},
$$

where

$$
U_{N} \equiv \frac{\sin (N+1) \varphi}{\sin \varphi},
$$

with $\varphi \equiv \cos ^{-1} z$ and $z \equiv(1 / 2)$ tr $\mathbf{M}$, being Chebyshev polynomials of the second kind satisfying the recursion relation:

$$
U_{n+1}-2 z U_{n}+U_{n-1}=0, \quad n \geq 1,
$$

with $U_{0}(z)=1$ and $U_{1}(z)=2 z$.

18. In an analogous way, the condition $\mathbf{B}^{3}= \pm \mathbf{B}$ reduces the global transfer matrix of the original triadic Cantor lattice to that corresponding to a periodic structure with a unit cell $A B$ (plus an isolated $A$ at the right end). On the contrary, the condition $\mathbf{B}^{3}= \pm \mathbf{A}$ transforms the original global transfer matrix into that corresponding to the nonhomogeneous fractal lattice $A B A^{3} B A^{5} B A^{3} B A \cdots$.

19. A more distinctive lattice-like structure has been reported for the wave function amplitudes distribution of certain states in Thue-Morse lattices [74].
20. Note that in the critical wave functions considered in the Aubry-André model the self-similar property extends over the entire support of the function rather than on a limited interval of it.

21. Note that for the global transfer matrices of the form

$$
\left(\begin{array}{cc}
0 & t_{B}^{-(2 m+1)} \\
t_{B}^{2 m+1} & 0
\end{array}\right),
$$

one obtains the eigenvalues $\lambda_{ \pm}= \pm 1$.

22. Note that (72) is invariant under the inversion operation $t_{B} \rightarrow t_{B}^{-1}$.

\section{Acknowledgments}

It is with great pleasure that the author thanks Professor Arunava Chakrabarti for many insightful conversations and useful comments on the nature of states in aperiodic systems during the last two decades. He/she also thank Professor Luca Dal Negro, Professor Uichiro Mizutani, Professor David Damanik, Professor Roland Ketzmerick, and Dr. Stefanie Thiem for sharing with him/her useful information. The author thanks Victoria Hernández for a careful critical reading of the paper.

\section{References}

[1] D. Bohm, "Quantum theory as an indication of a new order in physics-part A. The development of new orders as shown through the history of physics," Foundations of Physics, vol. 1, no. 4, pp. 359-381, 1971.

[2] D. Shechtman, I. Blech, D. Gratias, and J. W. Cahn, "Metallic phase with long-range orientational order and no translational symmetry," Physical Review Letters, vol. 53, no. 20, pp. 1951-1953, 1984.

[3] D. Levine and P. J. Steinhardt, "Quasicrystals: a new class of ordered structures," Physical Review Letters, vol. 53, no. 26, pp. 2477-2480, 1984.

[4] "ICrU Report of the Executive Committee for 1991," Acta Crystallographica A, vol. 48, p. 922, 1992.

[5] T. Janssen, G. Chapuis, and M. de Boissieu, Aperiodic Crystals: From Modulated Phases to Quasicrystals, Oxford University Press, Oxford, UK, 2007.

[6] M. Baake, Quasicrystals: An Introduction to Structure, Physical Properties, and Applications, edited by J. B. Suck, M. Schreiber, and P. Häussler, Springer, Berlin, Germany, 2002.

[7] W. Steurer and S. Deloudi, Crystallography of QuasicrystalsConcepts, Methods and Structures, vol. 126 of Springer Series in Materials Science, Springer, Berlin, Germany, 2009.

[8] S. Ostlund and R. Pandit, "Renormalization-group analysis of the discrete quasiperiodic Schrödinger equation," Physical Review B, vol. 29, no. 3, pp. 1394-1414, 1984.

[9] U. Mizutani, Introduction to the Electron Theory of Metals, Cambridge University Press, Cambridge, UK, 2001.

[10] R. del Rio, S. Jitomirskaya, Y. Last, and B. Simon, "What is localization?" Physical Review Letters, vol. 75, no. 1, pp. 117-119, 1995.

[11] D. H. Dunlap, H. L. Wu, and P. W. Phillips, "Absence of localization in a random-dimer model," Physical Review Letters, vol. 65 , no. 1, pp. 88-91, 1990. 
[12] A. Sánchez, E. Maciá, and F. Domínguez-Adame, "Suppression of localization in Kronig-Penney models with correlated disorder," Physical Review B, vol. 49, p. 147, 1994.

[13] S. Ostlund, R. Pandit, D. Rand, H. J. Schellnhuber, and E. D. Siggia, "One-dimensional Schrödinger equation with an almost periodic potential," Physical Review Letters, vol. 50, no. 23, pp. 1873-1876, 1983.

[14] N. Mott and W. D. Twose, "The theory of impurity conduction," Advances in Physics, vol. 10, p. 107, 1961.

[15] E. Maciá, F. Domínguez-Adame, and A. Sánchez, "Energy spectra of quasiperiodic systems via information entropy," Physical Review E, vol. 50, p. 679, 1994.

[16] J. Bellissard, A. Bovier, and J. M. Ghez, Differential Equations with Application to Mathematical Physics, edited by W. F. Ames, E. M. Harrell II , and J. V. Herod, Academic Press, Boston, Mass, USA, 1993.

[17] D. Damanik, M. Embree, and A. Gorodetski, "Spectral properties of Schrödinger operators arising in the study of quasicrystals," Tech. Rep. TR12-21, Rice University CAAM Department, 2012.

[18] L. Dal Negro, N. Lawrence, J. Trevino, and G. Walsh, "Aperiodic order for nanophotonics," in Optics of Aperiodic Structures: Fundamentals and Device Applications, Pan Stanford, Boca Raton, Fla, USA, 2014.

[19] J. Bellissard, B. Iochum, and D. Testard, "Continuity properties of the electronic spectrum of 1D quasicrystals," Communications in Mathematical Physics, vol. 141, no. 2, pp. 353-380, 1991.

[20] J. Bellissard, A. Bovier, and J. M. Ghez, "Gap labelling theorems for one dimensional discrete schrödinger operators," Reviews in Mathematical Physics, vol. 4, p. 1, 1992.

[21] J. M. Luck, "Cantor spectra and scaling of gap widths in deterministic aperiodic systems," Physical Review B, vol. 39, no. 9, pp. 5834-5849, 1989.

[22] E. Maciá, "The role of aperiodic order in science and technology," Reports on Progress in Physics, vol. 69, p. 397, 2006.

[23] M. Dulea, M. Johansson, and R. Riklund, "Localization of electrons and electromagnetic waves in a deterministic aperiodic system," Physical Review B, vol. 45, no. 1, pp. 105-114, 1992.

[24] M. Dulea, M. Johansson, and R. Riklund, “Trace-map invariant and zero-energy states of the tight-binding Rudin-Shapiro model," Physical Review B, vol. 46, no. 6, pp. 3296-3304, 1992.

[25] M. Dulea, M. Johansson, and R. Riklund, "Unusual scaling of the spectrum in a deterministic aperiodic tight-binding model," Physical Review B, vol. 47, no. 14, pp. 8547-8551, 1993.

[26] A. Süto, "Singular continuous spectrum on a cantor set of zero Lebesgue measure for the Fibonacci Hamiltonian," Journal of Statistical Physics, vol. 56, no. 3-4, pp. 525-531, 1989.

[27] J. Bellissard, B. Iochum, E. Scoppola, and D. Testard, "Spectral properties of one dimensional quasi-crystals," Communications in Mathematical Physics, vol. 125, no. 3, pp. 527-543, 1989.

[28] B. Iochum and D. Testard, "Power law growth for the resistance in the Fibonacci model," Journal of Statistical Physics, vol. 65, no. 3-4, pp. 715-723, 1991.

[29] J. Bellissard, A. Bovier, and J.-M. Ghez, "Spectral properties of a tight binding Hamiltonian with period doubling potential," Communications in Mathematical Physics, vol. 135, no. 2, pp. 379-399, 1991.

[30] A. Bovier and J. M. Ghez, "Spectral properties of onedimensional Schrödinger operators with potentials generated by substitutions," Communications in Mathematical Physics, vol. 158, p. 45, 1993, Erratum in Communications in Mathematical Physics vol. 166, pp. 431, 1994.
[31] A. Bovier and J.-M. Ghez, "Remarks on the spectral properties of tight-binding and Kronig-Penney models with substitution sequences," Journal of Physics A, vol. 28, no. 8, pp. 2313-2324, 1995.

[32] S. Aubry and S. André, "Analyticity breaking and Anderson localization in in commensurate lattices," Annals of the Israel Physical Society, vol. 3, p. 133, 1980.

[33] H. Aoki, "Decimation method of real-space renormalization for electron systems with application to random systems," Physica $A$, vol. 114, no. 1-3, pp. 538-542, 1982.

[34] H. Aoki, "Critical behaviour of extended states in disordered systems," Journal of Physics C, vol. 16, no. 6, pp. L205-L208, 1983.

[35] C. M. Soukoulis and E. N. Economou, "Fractal character of eigenstates in disordered systems," Physical Review Letters, vol. 52, no. 7, pp. 565-568, 1984.

[36] C. Castellani and L. Peliti, "Multifractal wavefunction at the localisation threshold," Journal of Physics A, vol. 19, no. 8, article no. 004, pp. L429-L432, 1986.

[37] M. Schreiber, "Fractal character of eigenstates in weakly disordered three-dimensional systems," Physical Review B, vol. 31, no. 9, pp. 6146-6149, 1985.

[38] H. Aoki, "Fractal dimensionality of wave functions at the mobility edge: quantum fractal in the Landau levels," Physical Review B, vol. 33, no. 10, pp. 7310-7313, 1986.

[39] Y. Oono, T. Ohtsuki, and B. Kramer, "Inverse participation number and fractal dimensionality of electronic states in a two dimensional system in strong perpendicular magnetic field," Journal of the Physical Society of Japan, vol. 58, pp. 1705-1716, 1989.

[40] H. Grussbach and M. Schreiber, "Multifractal electronic wave functions in the Anderson model of localization," Modern Physics Letters, vol. 6, p. 851, 1992.

[41] D. R. Hofstadter, "Energy levels and wave functions of Bloch electrons in rational and irrational magnetic fields," Physical Review B, vol. 14, no. 6, pp. 2239-2249, 1976.

[42] M. Kohmoto, "Localization problem and mapping of onedimensional wave equations in random and quasiperiodic media," Physical Review B, vol. 34, no. 8, pp. 5043-5047, 1986.

[43] S. N. Evangelou, "Multi-fractal spectra and wavefunctions of one-dimensional quasi-crystals," Journal of Physics C, vol. 20, p. L295, 1987.

[44] S. Mugabassi and A. Vourdas, "Localized wavefunctions in quantum systems with multiwell potentials," Journal of Physics A, vol. 43, Article ID 325304, 2010.

[45] E. Maciá and F. Domínguez-Adame, "Three-dimensional effects on the electronic structure of quasiperiodic systems," Physica B, vol. 216, p. 53, 1995.

[46] E. Maciá Barber, Aperiodic Structures in Condensed Matter, CRC Taylor \& Francis, Boca Raton, Fla, USA, 2009.

[47] F. Domínguez-Adame, E. Maciá, B. Méndez, C. L. Roy, and A. Khan, "Fibonacci superlattices of narrow-gap III-V semiconductors," Semiconductor Science and Technology, vol. 10, p. 797, 1995.

[48] M. Kohmoto, B. Sutherland, and C. Tang, "Critical wave functions and a Cantor-set spectrum of a one-dimensional quasicrystal model," Physical Review B, vol. 35, no. 3, pp. 1020 1033, 1987.

[49] E. Maciá and F. Domínguez-Adame, "Physical nature of critical wave functions in Fibonacci systems," Physical Review Letters, vol. 76, p. 2957, 1996. 
[50] V. Kumar, "Extended electronic states in a Fibonacci chain," Journal of Physics, vol. 2, no. 5, article no. 026, pp. 1349-1353, 1990.

[51] R. Rammal, "Nature of eigenstates on fractal structures," Physical Review B, vol. 28, no. 8, pp. 4871-4874, 1983.

[52] W. A. Schwalm and M. K. Schwalm, "Electronic properties of fractal-glass models," Physical Review B, vol. 39, pp. 1287212882, 1989.

[53] W. A. Schwalm and M. K. Schwalm, "Explicit orbits for renormalization maps for Green functions on fractal lattices," Physical Review B, vol. 47, no. 13, pp. 7847-7858, 1993.

[54] R. F. S. Andrade and H. J. Schellnhuber, "Electronic states on a fractal: exact Green's-function renormalization approach," Physical Review B, vol. 44, no. 24, pp. 13213-13227, 1991.

[55] E. Maciá, "Electronic transport in the Koch fractal lattice," Physical Review B, vol. 57, no. 13, pp. 7661-7665, 1998.

[56] P. Kappertz, R. F. S. Andrade, and H. J. Schellnhuber, "Electronic states on a fractal: inverse-iteration method," Physical Review B, vol. 49, no. 20, pp. 14711-14714, 1994.

[57] Z. Lin and M. Goda, "Power-law eigenstates of a regular Vicsek fractal with hierarchical interactions," Physical Review B, vol. 50, no. 14, pp. 10315-10318, 1994.

[58] A. Chakrabarti, "Field induced delocalization in a Koch fractal," Physical Review B, vol. 60, no. 15, pp. 10576-10579, 1999.

[59] A. Chakrabarti, "Exact results for infinite and finite Sierpinski gasket fractals: extended electron states and transmission properties," Journal of Physics, vol. 8, no. 50, pp. 10951-10957, 1996.

[60] S. Sengupta, A. Chakrabarti, and S. Chattopadhyay, "Electronic properties of a Cantor lattice," Physica B, vol. 344, no. 1-4, pp. 307-318, 2004.

[61] A. Chakrabarti and B. Bhattacharyya, "Atypical extended electronic states in an infinite Vicsek fractal: an exact result," Physical Review B, vol. 54, no. 18, pp. R12625-R12628, 1996.

[62] A. Chakrabarti and B. Bhattacharyya, "Sierpinski gasket in a magnetic field: electron states and transmission characteristics," Physical Review B, vol. 56, no. 21, pp. 13768-13773, 1997.

[63] C. S. Jayanthi and S. Y. Wu, "Frequency spectrum of a general Vicsek fractal," Physical Review B, vol. 48, no. 14, pp. 1018810198, 1993.

[64] Y. E. Lévy and B. Souillard, "Superlocalization of electrons and waves in fractal media," Europhysics Letters, vol. 4, p. 233, 1987.

[65] S. Sengupta, A. Chakrabarti, and S. Chattopadhyay, "Electronic transport in a Cantor stub waveguide network," Physical Review B, vol. 72, no. 13, Article ID 134204, 2005.

[66] R. F. S. Andrade and H. J. Schellnhuber, "Exact treatment of quantum states on a fractal," Europhysics Letters, vol. 10, p. 73, 1989.

[67] Y. Gefen, B. B. Mandelbrot, and A. Aharony, "Critical phenomena on fractal lattices," Physical Review Letters, vol. 45, no. 11, pp. 855-858, 1980.

[68] E. Maciá, "Exploiting aperiodic designs in nanophotonic devices," Reports on Progress in Physics, vol. 75, Article ID 036502, 2012.

[69] L. Dal Negro and S. V. Boriskina, "Deterministic aperiodic nanostructures for photonics and plasmonics applications," Laser and Photonics Reviews, vol. 6, no. 2, pp. 178-218, 2012.

[70] E. Maciá, “The importance of being aperiodic: optical devices," in Optics of Aperiodic Structures: Fundamentals and Device Applications, Pan Stanford, Boca Raton, Fla, USA, 2014.

[71] Z. Valy Vardeny, A. Nahata, and A. Agrawal, "Optics of photonic quasicrystals," Nature Photonics, vol. 7, p. 177, 2013.
[72] S. V. Gaponenko, Introduction to Nanophotonics, Cambridge University Press, Cambridge, UK, 2010.

[73] M. Severin, M. Dulea, and R. Riklund, "Periodic and quasiperiodic wavefunctions in a class of one-dimensional quasicrystals: an analytical treatment," Journal of Physics, vol. 1, no. 45, pp. 8851-8858, 1989.

[74] C. S. Ryu, G. Y. Oh, and M. H. Lee, "Extended and critical wave functions in a Thue-Morse chain," Physical Review B, vol. 46, no. 9, pp. 5162-5168, 1992.

[75] W. Gellermann, M. Kohmoto, B. Sutherland, and P. C. Taylor, "Localization of light waves in Fibonacci dielectric multilayers," Physical Review Letters, vol. 72, no. 5, pp. 633-636, 1994.

[76] A. Chakrabarti, S. N. Karmakar, and R. K. Moitra, "Renormalization-group analysis of extended electronic states in one-dimensional quasiperiodic lattices," Physical Review B, vol. 50, no. 18, pp. 13276-13285, 1994.

[77] V. Kumar and G. Ananthakrishna, "Electronic structure of a quasiperiodic superlattice," Physical Review Letters, vol. 59, no. 13, pp. 1476-1479, 1987.

[78] X. C. Xie and S. Das Sarma, "Extended electronic states in a Fibonacci superlattice," Physical Review Letters, vol. 60, no. 15, p. $1585,1988$.

[79] S. Sil, S. N. Karmakar, R. K. Moitra, and A. Chakrabarti, "Extended states in one-dimensional lattices: application to the quasiperiodic copper-mean chain," Physical Review B, vol. 48, no. 6, pp. 4192-4195, 1993.

[80] M. Kohmoto and J. R. Banavar, "Quasiperiodic lattice: electronic properties, phonon properties, and diffusion," Physical Review B, vol. 34, no. 2, pp. 563-566, 1986.

[81] Q. Niu and F. Nori, "Renormalization-group study of onedimensional quasiperiodic systems," Physical Review Letters, vol. 57, no. 16, pp. 2057-2060, 1986.

[82] Q. Niu and F. Nori, "Spectral splitting and wave-function scaling in quasicrystalline and hierarchical structures," Physical Review $B$, vol. 42, no. 16, pp. 10329-10341, 1990.

[83] G. J. Jin and Z. D. Wang, "Are self-similar states in Fibonacci systems transparent?" Physical Review Letters, vol. 79, p. 5298, 1997.

[84] B. Sutherland and M. Kohmoto, "Resistance of a onedimensional quasicrystal: power-law growth," Physical Review B, vol. 36, no. 11, pp. 5877-5886, 1987.

[85] S. Das Sarma and X. C. Xie, "Conductance fluctuations in onedimensional quasicrystals," Physical Review B, vol. 37, no. 3, pp. 1097-1102, 1988.

[86] J. M. Dubois, Useful Quasicrystals, World Scientific, Singapore, 2005.

[87] X. Huang and C. Gong, "Property of Fibonacci numbers and the periodiclike perfectly transparent electronic states in Fibonacci chains," Physical Review B, vol. 58, no. 2, pp. 739-744, 1998.

[88] D. Kalman and R. Mena, "The Fibonacci numbers: exposed," Mathematics Magazine, vol. 76, pp. 167-181, 2003.

[89] R. Oviedo-Roa, L. A. Pérez, and C. Wang, "Ac conductivity of the transparent states in Fibonacci chains," Physical Review B, vol. 62, no. 21, pp. 13805-13808, 2000.

[90] V. Sánchez, L. A. Pérez, R. Oviedo-Roa, and C. Wang, "Renormalization approach to the Kubo formula in Fibonacci systems," Physical Review B, vol. 64, Article ID 174205, 2001.

[91] V. Sánchez and C. Wang, "Application of renormalization and convolution methods to the Kubo-Greewood formula in multidimensional Fibonacci systems," Physical Review B, vol. 70, Article ID 144207, 2004. 
[92] S. Thiem and M. Schreiber, "Wavefunctions, quantum diffusion, and scaling exponents in golden-mean quasiperiodic tilings," Journal of Physics, vol. 25, Article ID 075503, 2013.

[93] H. Bohr, Collected Mathematical Works-II Almost Periodic Functions, Dansk Matematisk Forening, Copenhagen, Denmark, 1952.

[94] F. R. Gantmacher, The Theory of Matrices 2, Chelsea, New York, NY, USA, 1974. 

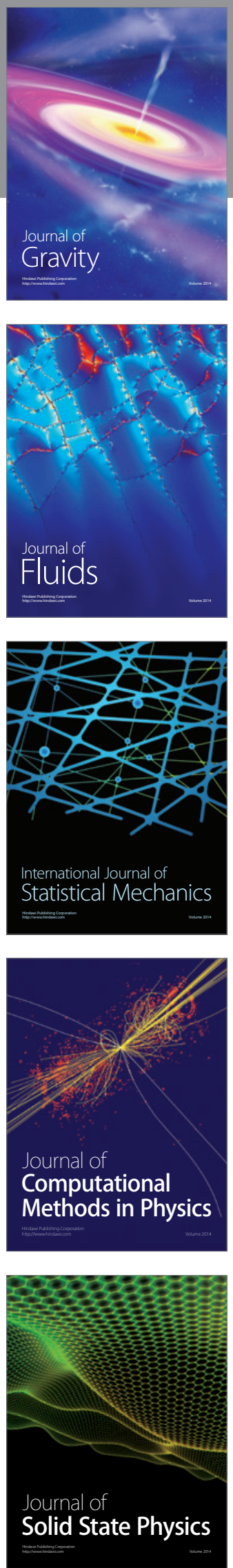

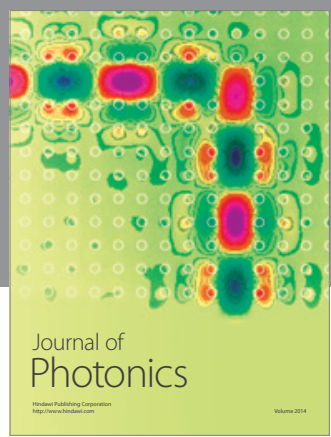

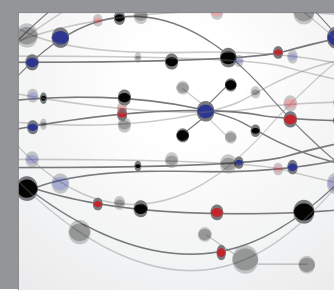

The Scientific World Journal

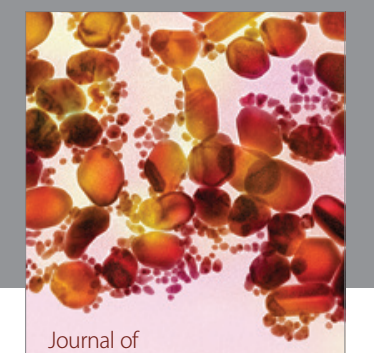

Soft Matter
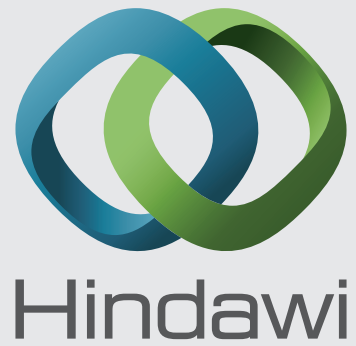

Submit your manuscripts at

http://www.hindawi.com
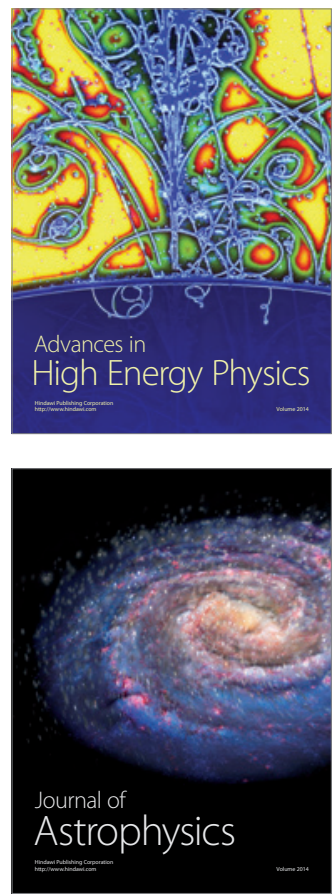
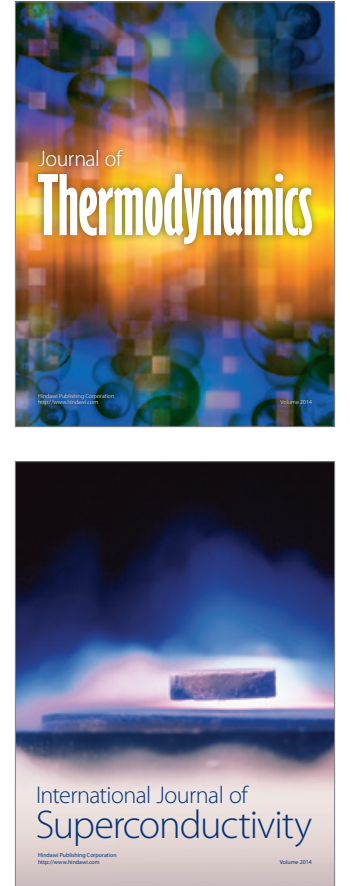
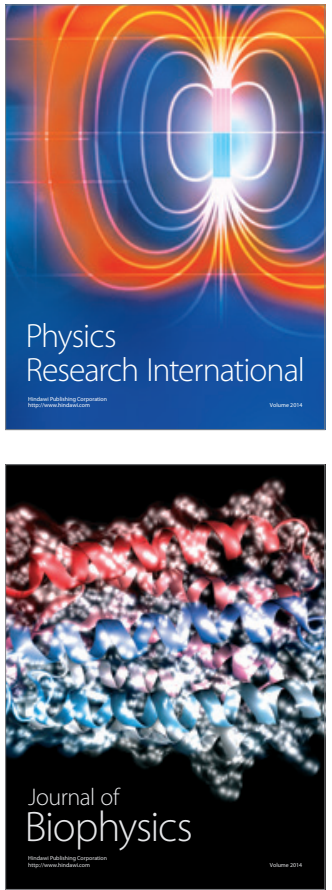
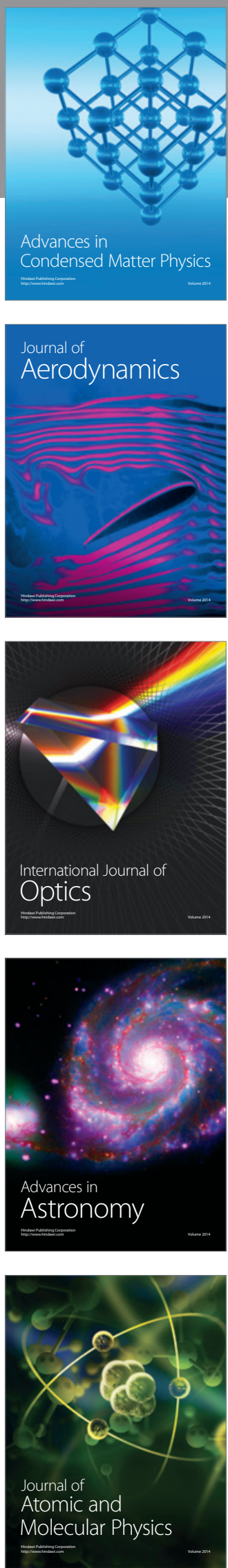